Draft version July 17, 2021, ACCePted By the Astronomical Journal

Preprint typeset using $\mathrm{LATEX}_{\mathrm{E}}$ style emulateapj v. 11/10/09

\title{
THE CELESTIAL REFERENCE FRAME AT 24 AND 43 GHZ. II. IMAGING
}

\author{
P. Charlot $^{1,2}$, D. A. Boboltz ${ }^{3}$, A. L. FeY ${ }^{3}$, E. B. Fomalont ${ }^{4}$, B. J. Geldzahler ${ }^{5}$,

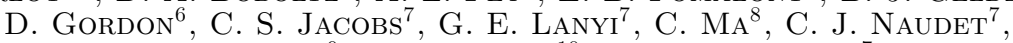 \\ J. D. Romney ${ }^{9}$, O. J. Sovers ${ }^{10}$, ANd L. D. Zhang ${ }^{7}$ \\ Draft version July 17, 2021, accepted by the Astronomical Journal
}

\begin{abstract}
We have measured the sub-milli-arcsecond structure of 274 extragalactic sources at 24 and $43 \mathrm{GHz}$ in order to assess their astrometric suitability for use in a high frequency celestial reference frame (CRF). Ten sessions of observations with the Very Long Baseline Array have been conducted over the course of $\sim 5$ years, with a total of 1339 images produced for the 274 sources. There are several quantities that can be used to characterize the impact of intrinsic source structure on astrometric observations including the source flux density, the flux density variability, the source structure index, the source compactness, and the compactness variability. A detailed analysis of these imaging quantities shows that (1) our selection of compact sources from $8.4 \mathrm{GHz}$ catalogs yielded sources with flux densities, averaged over the sessions in which each source was observed, of about 1 Jy at both 24 and $43 \mathrm{GHz}$, (2) on average the source flux densities at $24 \mathrm{GHz}$ varied by $20 \%-25 \%$ relative to their mean values, with variations in the session-to-session flux density scale being less than $10 \%,(3)$ sources were found to be more compact with less intrinsic structure at higher frequencies, and (4) variations of the core radio emission relative to the total flux density of the source are less than $8 \%$ on average at $24 \mathrm{GHz}$. We conclude that the reduction in the effects due to source structure gained by observing at higher frequencies will result in an improved CRF and a pool of high-quality fiducial reference points for use in spacecraft navigation over the next decade.
\end{abstract}

Subject headings: astrometry — quasars: general — radio continuum: galaxies — surveys

\section{INTRODUCTION}

The International Celestial Reference Frame (ICRF) was formally adopted as the fundamental celestial reference frame (CRF) by the International Astronomical Union (IAU) in 1997. The catalog includes precise astrometric positions of over 600 extragalactic compact radio sources distributed uniformly over the sky. These positions were determined from the analysis of thousands of dual-frequency S/X-band (2.3/8.4 GHz) Very Long Baseline Interferometry (VLBI) observational sessions recorded between 1979 and 1995. The frame itself was defined by the 212 highest-quality "defining" sources with typical position accuracies of 0.25 milli-arcseconds (mas), while the axes of the frame are accurate to 0.02 mas (Ma et al. 1998). In addition to the 212 defining sources, positions for 294 less observed "candidate" sources along with 102 "other" sources with excessive po-

\footnotetext{
${ }^{1}$ Université de Bordeaux, Observatoire Aquitain des Sciences de l'Univers, BP 89, 33271 Floirac Cedex, France

2 CNRS, Laboratoire d'Astrophysique de Bordeaux UMR 5804, BP 89, 33271 Floirac Cedex, France

${ }^{3}$ U.S. Naval Observatory, 3450 Massachusetts Ave., NW, Washington, DC 20392-5420

${ }^{4}$ National Radio Astronomy Observatory, 520 Edgemont Rd., Charlottesville, VA 22903

${ }^{5}$ National Aeronautics and Space Administration, 300 E. St., SW, Washington, DC 20546

6 NVI Inc./NASA Goddard Space Flight Center, Greenbelt, MD 20771

7 Jet Propulsion Laboratory, Caltech, 4800 Oak Grove Dr., Pasadena, CA 91109

${ }^{8}$ NASA Goddard Space Flight Center, Greenbelt, MD 20771

${ }^{9}$ National Radio Astronomy Observatory, P.O. Box O, Socorro, NM 87801

10 Remote Sensing Analysis Systems, 2092 Sinaloa Ave., Altadena, CA 91001
}

sition variation, were also given to increase the density of the frame (Ma et al. 1998). Since its adoption, incremental updates to the catalog of sources have occurred in the form of two extensions to the ICRF (Fey et al. 2004) using hundreds of additional sessions along with improved analysis and modeling techniques. However, the positions of the original 212 defining sources have remained constant through these extensions.

The compact extragalactic radio sources that comprise the ICRF have been the subject of extensive study since the inception of VLBI techniques. In the standard theory, (e.g. Blandford \& Königl 1979), the jet-like emission from quasars and active galactic nuclei is assumed to be powered by a central engine where energetic phenomena occur. The observed, frequency dependent, intrinsic structure of extragalactic radio sources typically consists of a flat spectrum $\left(S \propto \nu^{\alpha}, \alpha \approx 0\right)$ unresolved core at the base of the jet where the optical depth is near unity $(\tau \approx 1)$ and extended emission in the form of multiple steep spectrum $(\alpha \approx-0.5$ to -1.5$)$ jet components. These components often move away from the core along the direction of the jet, sometimes at apparent superluminal speeds. The emission outside of the core has been shown to be extended on scales larger than the accuracy of the astrometric position measurements (Fey et al. 1996). This extended emission, or intrinsic source structure, contributes to the uncertainty in the measured astrometric positions of sources that comprise the ICRF. In addition to extended emission, frequency dependent opacity effects can contribute to variations in the measured astrometric positions of extragalactic radio sources. In particular, opacity conditions in the region near the base of the jet can cause the measured core position to move inward along the jet direction as a function of in- 
creasing radio frequency of the observations. Such core shifts have been measured for some compact sources (e.g. Lobanov 1998; Kovalev et al. 2008).

Based upon the discussion above, there are potential advantages to observing astrometric sources at radio frequencies higher than the typical $\mathrm{S} / \mathrm{X}$ band observations. First, the resolution of the observations is increased, thus improving the astrometric accuracy. Second, the effects due to intrinsic source structure may be reduced since the core and jet components have different spectral characteristics and the core is expected to be more dominant at high frequencies. We have undertaken a program to observe a number of extragalactic sources at $\mathrm{K}$ band (24 $\mathrm{GHz}$ ) and $\mathrm{Q}$ band $(43 \mathrm{GHz})$ using the 10 stations of the Very Long Baseline Array (VLBA). At these higher frequencies, only the VLBA provides the stability, imaging capabilities and frequency coverage to enable such a program. The long term goals of the program include: 1) developing a high-frequency $\mathrm{CRF}$ with a variety of applications including improved deep space navigation, 2) providing the astronomical community with an extended catalog of calibrator sources for VLBI observations at 24 and $43 \mathrm{GHz}$, and 3) studying the effects of the intrinsic structure of extragalactic sources to improve the astrometric accuracy of future high-frequency reference frames. A detailed discussion of the program and the astrometric results is contained in a companion paper (Lanyi et al. 2009, hereafter Paper I). In this paper, we concentrate on the imaging aspects of the program and the effects of observed source structure and variability on astrometric accuracy.

Theoretically, an optimal CRF would be composed of sources with strong, non-variable, point-like emission. In reality, however, virtually all sources possess some structure and intrinsic variability in the emission over time. In addition, there is the potential for sudden flaring events even for normally quiescent sources. Thus it is highly desirable to characterize and monitor the nature of the sources used in a CRF through periodic VLBI imaging. The VLBA has previously been used to make simultaneous dual-frequency S/X-band $(2.3 / 8.4$ $\mathrm{GHz}$ ) observations of a total of 389 ICRF sources (Fey et al. 1996; Fey \& Charlot 1997, 2000). To date, approximately $90 \%$ of the ICRF sources north of $-20^{\circ}$ declination have been imaged at least once at both 2.3 and $8.4 \mathrm{GHz}$. Based on the initial work of Charlot (1990), the database of VLBA X-band and S-band images was analyzed by Fey \& Charlot (1997, 2000) in order to quantitatively improve our understanding of the relationship between extended source structure and the astrometric positions determined from VLBI. Here we discuss our growing database of high-frequency images of potential extragalactic reference frame sources. We apply similar analysis techniques in an attempt to characterize the impact of extended source structure on the astrometric accuracy of the catalog of source positions obtained from our VLBA high-frequency data (Paper I).

\section{OBSERVATIONS AND DATA REDUCTION}

We observed a total of 351 extragalactic radio sources using the 10 antennas of the VLBA (Napier et al. 1994) of the National Radio Astronomy Observatory (NRAO) ${ }^{11}$ over the course of 10 sessions spanning five years from 2002-2007. In all 10 of the sessions, observations were recorded at $\mathrm{K}$-band $(24 \mathrm{GHz})$. Additionally, in four of the 10 sessions, observations were also recorded at Qband $(43 \mathrm{GHz})$. In two of the sessions, dual S/X-band $(2.3 / 8.4 \mathrm{GHz})$ observations were interspersed with the K-band observations to investigate potential ionospheric correction methods. A summary of the VLBA K- and Q-band observations relevant to the imaging program is presented in Table 1 .

The frequency information listed in Table 1 reflects the changing observing strategy over the course of the 10 VLBA sessions. For the first two sessions, four $8 \mathrm{MHz}$ bands each at 24 and $43 \mathrm{GHz}$ were recorded simultaneously for each scan using 2 bit sampling yielding a total bandwidth of $32 \mathrm{MHz}$. The remaining sessions used eight $8 \mathrm{MHz}$ bands with 1 bit sampling for a total bandwidth of $64 \mathrm{MHz}$. For these sessions, scans alternated between $\mathrm{K}$ and $\mathrm{Q}$ bands (sessions 3 and 5), simultaneous S/X band and $\mathrm{K}$ band (sessions 6 and 8 ), or just $\mathrm{K}$ band alone (sessions 4, 9, and 10). The first K-only session observed a large number of potential sources found in other VLBI surveys in order to expand the total number of available high-frequency astrometric targets. In the final two Konly sessions, candidate sources near the ecliptic plane were added for potential future use in deep space navigation. A detailed discussion of the evolving observing strategy and its relevance to the astrometric goals of the program is presented in Paper I.

All of the observations were made in a bandwidth synthesis mode to facilitate group delay measurements for astrometry - the multiplicity of channels allows for the determination of a precise group delay (Rogers 1970). Observations in this mode also allow snapshot imaging. Source scans were typically 2 minutes in duration at $\mathrm{K}$ band and 3 minutes for $\mathrm{Q}$ band. Most sources were observed in three or more scans - the one exception being the K-band survey session on 2003 May 22 in which many of the sources were observed only once or twice. The raw data bits were correlated with the VLBA correlator at the Array Operations Center in Socorro, New Mexico.

The correlated data were calibrated and corrected for residual delay and delay rate using the NRAO's Astronomical Image Processing System (AIPS). Initial amplitude calibration for each intermediate frequency (IF) was accomplished using system temperature measurements taken during the observations combined with NRAO supplied gain curves. Fringe-fitting was done in AIPS using solution intervals equal to the scan durations and a point source model in all cases. After correction for residual delay and delay rate, the data were written to FITS disk files. All subsequent image processing was carried out using the Caltech VLBI imaging software, primarily DIFMAP.

The visibility data for each frequency band were self-calibrated, Fourier inverted, and CLEANed using DIFMAP in an automatic mode (Pearson et al. 1994). DIFMAP combines the visibilities for each $1 \mathrm{~F}$ of an observation in the $(u, v)$-plane during gridding, taking into

11 The National Radio Astronomy Observatory is a facility of the National Science Foundation operated under cooperative agreement by Associated Universities, Inc. 
account frequency differences. However, DIFMAP makes no attempt to correct for spectral index effects. The spanned bandwidth of the IFs in each band is relatively small $(0.5 \mathrm{GHz}-2 \%$ of the fractional bandwidth), so we assume that source structure and flux density variations across each of the two frequency bands are negligible. After phase self-calibration with a point source model, the $4 \mathrm{~s}$ correlator records were coherently averaged to 12 $\mathrm{s}$ records. The averaged data were subsequently edited to remove outliers due to poor data and times in which the antennas were not on source.

The amplitude calibration within each particular session at each frequency band was improved through observations of a strong, compact source. A single amplitude gain correction factor was derived for each antenna for each IF, based on fitting a simple Gaussian source model to the visibility data of these compact sources after applying only the initial calibration based on the measured system temperatures and gain curves. Gain correction factors were calculated based on the differences between the observed and model visibilities. The resulting amplitude gain correction factors were typically less than $5 \%$ and were applied to the visibility data of all sources.

The data were self-calibrated following the hybridmapping technique (Pearson \& Readhead 1984) to correct for residual amplitude and phase errors. The data were initially phase self-calibrated and mapped using uniform weighting in the $u, v$-plane before switching to natural weighting after several iterations. A point source model was used as a starting model for the iterative procedure in all cases. Convergence was defined basically as the iteration when the peak in the residual image became less than about six to eight times the root-mean-square (rms) noise of the residual image from the previous iteration. The $1 \%-2 \%$ of sources with emission structure too complex or too extended for the automatic imaging script to handle were imaged by hand, i.e. in an interactive mode, following the same prescription as that for the automatic mode. Convergence for these sources was subjective and was based on the iteration at which it was judged that further self-calibration would not significantly improve the resultant image.

Subsequent to the calibration and imaging process, an analysis of the variations in the flux density scaling from one session to the next was performed using 65 sources observed in at least 6 of 10 sessions at $K$ band and 31 sources observed in at least 3 of 4 sessions at Q band. For this analysis, the mean and standard deviation of the flux density for the ensemble of sources at each band were computed. The results showed that, on average, the relative flux density scaling from one session to the next varied by $\sim 5 \%$ at $\mathrm{K}$ band and $\sim 9 \%$ at $\mathrm{Q}$ band.

\section{IMAGING RESULTS AND ANALYSIS}

Table 1 provides a summary of the number of sources successfully imaged (out of the 351 total) from the 10 sessions of K-band observations and four sessions of Q-band observations recorded as part of the high-frequency CRF program. The results of the imaging part of the program are presented graphically in the form of contour plots available via the USNO Radio Reference Frame Image Database (RRFID) ${ }^{12}$, Shown in Figures 1 and 2 are

12 http://rorf.usno.navy.mil/rrfid.shtml example contour maps from the most recent session of each of the 274 sources imaged at $\mathrm{K}$ band and of each of the 132 sources imaged at Q band. For convenience, the resulting K-band maps are identified by only a single fiducial frequency (either $\sim 23.9$ or $24.4 \mathrm{GHz}$ depending on session) even though they come from data using all frequency channels. Similarly, the Q-band maps are also identified by only a single fiducial frequency $(\sim 43.1 \mathrm{GHz})$.

Tables 2 and 3 list the parameters relevant to the final images at each frequency including: the observation session, the elliptical beam size and position angle, the peak flux density $\left(S_{\text {peak }}\right)$, the total flux density $\left(S_{\text {total }}\right)$, the rms of the residuals of the flux density $\left(S_{\mathrm{rms}}\right)$, and the contour levels in the maps shown in Figures 1 and 2 . The total flux density is the sum of all CLEAN component model flux densities obtained from the source images (Clark 1980). From the data in the table, we determined the minimum, maximum and average dynamic range $\left(S_{\text {peak }} / S_{\text {rms }}\right)$ for all of the K-band images to be $\sim 40: 1, \sim 1720: 1$, and $\sim 340: 1$, respectively. The average $\mathrm{rms}$ noise at $\mathrm{K}$ band is $\sim 2.8 \mathrm{mJy}^{\mathrm{beam}^{-1}}$. The minimum, maximum, and average dynamic range for the Q-band images are $\sim 60: 1, \sim 910: 1$, and $\sim 250: 1$, respectively. The average $\mathrm{rms}$ noise at $\mathrm{Q}$ band is $\sim 5.0 \mathrm{mJy}^{\text {beam }^{-1}}$. For comparison, the expected thermal noise at $\mathrm{K}$ band assuming 6 minutes on source $(\sim 2$ minutes per scan times three scans) is estimated to be $\sim 0.7 \mathrm{mJy} \mathrm{beam}^{-1}$. At $\mathrm{Q}$ band, assuming 9 minutes on source $(\sim 3$ minutes per scan times three scans) the expected thermal noise is $\sim 1.6$ mJy beam $^{-1}$.

The CLEAN component models were also used to determine two additional parameters, the source structure index $(S I)$ and the source compactness $(C)$. The structure index is described in detail in $\S 3.2$. The source compactness is defined as the percentage of the total CLEANed flux density contained within a radius approximately equal to one synthesized beam width centered on the peak emission in the image.

\subsection{Source Flux Density}

For a source to be an effective astrometric reference (e.g. for spacecraft tracking and astronomical phase referencing) it must be detectable within a coherence time at the observing frequency; typically less than 2 minutes at $\mathrm{K}$ and $\mathrm{Q}$ bands. Sources should, therefore, have sufficient flux density for the given observational sensitivity, and sufficiently small variability so as not to fall below the detection threshold. From the CLEAN components models described above, the total source flux densities $\left(S_{\text {total }}\right)$ were determined for all of the sources in all sessions in both frequency bands. From $S_{\text {total }}$ we computed the mean flux density $(\bar{S})$ per source averaged over all sessions in which the source was observed. Plotted in Figure 3 are the distributions of $\bar{S}$ for all of the sources imaged in the program including 138 sources at X band, 274 sources at $\mathrm{K}$ band, and 132 sources at $\mathrm{Q}$ band. The mean (median) values of the distributions are approximately 1.5 (0.8) Jy at X band, 1.1 (0.7) Jy at K band, and $1.4(0.9)$ Jy at $\mathrm{Q}$ band. Shown in Figure 4 are the same distributions of $\bar{S}$ for only those 97 sources that are common to all three bands. Here the mean (median) values are $1.7(0.9), 1.5(0.9)$ and $1.5(1.0) \mathrm{Jy}$ at $\mathrm{X}, \mathrm{K}$ 
and Q bands, respectively.

It is not surprising that for the case of all sources and for the 97 sources in common that the mean flux densities at $\mathrm{X}$ band are the highest, especially since the strongest (> $300 \mathrm{mJy}$ ) most compact sources in the $\mathrm{S} / \mathrm{X}$-band ICRF program were selected for the K/Q-band observations in order to optimize the probability of detection at the higher frequencies (Paper I). This selection effect is also, very likely, the reason that, on average, the Q-band mean flux densities are larger than those at $\mathrm{K}$ band for all sources (Figure 3). The Q-band sources were drawn strictly from this pool of strong, compact, ICRF sources. However, as Table 1 shows, a significant fraction of the K-band sources were observed in a single-session survey that occurred on 2003 May 22. For this survey, the Xband flux density criterion was relaxed from a nominal value of $300 \mathrm{mJy}$ to $200 \mathrm{mJy}$ in order to include more sources. For the 97 sources in common to all three bands, the selection effect is reduced since many of the K-band survey sources are excluded. Figure 4 shows the K- and Q-band mean flux densities to be consistent with each other to within the $\sim 10 \%$ session-to-session flux density scale variations mentioned previously.

The extent to which the total flux density varies over time can be characterized by the flux density variability index $\left(\sigma_{S} / \bar{S}\right)$, which is the standard deviation of the measured flux densities divided by the mean. A minimum value, $\sigma_{S} / \bar{S}=0.0$, indicates no variation in the flux density with time. Since a number of the sources at $\mathrm{K}$ and $\mathrm{Q}$ bands were observed in multiple sessions, it is possible to determine $\sigma_{S} / \bar{S}$ for these sources. This was not the case for $\mathrm{X}$ band where only 25 sources were observed in both of the two X-band sessions. Distributions for the flux density variability index for the 235 sources at $\mathrm{K}$ band and 82 sources at Q band observed in two or more sessions are shown in Figure 5 . The mean and median values of $\sigma_{S} / \bar{S}$ at $\mathrm{K}$ band are 0.18 and 0.16 , respectively. The Q-band mean and median values of the flux density variability for sources observed in two or more sessions are 0.19 and 0.15 , respectively.

Given of the relatively few number of sessions thus far observed, the values above represent lower limits to the flux density variability. Increasing the cutoff for the minimum number of sessions per source to five, reduces the number of available K-band sources to 87 and yields a mean value of $\sigma_{S} / \bar{S}$ of 0.23 . Further increasing the minimum cutoff to 10 sessions, reduces the number of sources to 18 and results in a mean flux density variability of 0.24 . At $\mathrm{Q}$ band there were 22 sources with data available from all four observing sessions. The mean flux density variability for these 22 sources was 0.28 . Our preliminary results therefore indicate that on average the flux density varied by $20 \%-25 \%$ at $\mathrm{K}$ band, and $20 \%-30 \%$ at $\mathrm{Q}$ band. These results are limited to the $\sim 5$ year period over which our data were recorded and by the $\sim 10 \%$ variations in the flux density scaling mentioned previously.

In addition to the flux density variability index, we also determined the ratio of the maximum to minimum flux density $\left(S_{\max } / S_{\min }\right)$ for each source observed in more than one session. Distributions for $S_{\max } / S_{\min }$ at $\mathrm{K}$ band and $\mathrm{Q}$ band are plotted in Figure 6, with the mean and median ratio for each distribution indicated. For the two distributions, the mean (median) values are 1.7 (1.5) at $\mathrm{K}$ band, and 1.7 (1.4) at Q band. The figure shows that over the five-year period in which the sources were monitored, the maximum to minimum flux density ratio was greater than a factor of 2 for roughly $23 \%$ of the sources at $\mathrm{K}$ band and $24 \%$ of the sources at $\mathrm{Q}$ band. The ratio is greater than a factor of 3 for only $6 \%$ of the sources at $\mathrm{K}$ band and $4 \%$ of the sources at $\mathrm{Q}$.

Our flux density results can be compared to other extragalactic source monitoring programs. The $14-\mathrm{m}$ telescope at the Metsahovi Radio Observatory has monitored the flux densities of active galactic nuclei at 22, 37 , and $87 \mathrm{GHz}$ over the course of $25+$ years (Teräsranta et al. 2005, and reference therein). Statistical analysis of this data using several different methods (Hovatta et al. 2008, 2007) has shown that smaller flux density variations occur on shorter timescales of 1-2 years, while larger outbursts are rare occurring on average every 6 years. Their sample consisted of 80 sources with a minimum flux density of $1 \mathrm{Jy}$ and a minimum monitoring time of ten years. Although the time span of our data is shorter, the sampling much sparser, and the minimum flux density lower at 200-300 mJy, our K- and Q-band results seem to be consistent with the Metsahovi data. The variability index results show that the variations are relatively small over the five-year monitoring period, while the $S_{\max } / S_{\min }$ results indicate very few large-scale outbursts over the same time period.

\subsection{Source Structure Index}

As previously demonstrated by Charlot (1990), the contribution of intrinsic source structure to a VLBI bandwidth synthesis delay measurement can be significant. It depends on the exact form of the spatial brightness distribution of the extended radio source relative to the geometry of the VLBI baseline vector projected onto the plane of the sky. The overall magnitude of the source structure effect for a given source is most easily estimated by calculating corrections to the bandwidth synthesis delay based upon the observed source structure, for a range of $(u, v)$ coordinates $(u$ and $v$ are coordinates of the baseline vector projected onto the plane of the sky and are expressed in units of the observing wavelength). Following such a scheme, Fey \& Charlot (1997) defined a source "structure index" according to the median value of the structure delay corrections, $\tau_{\text {median }}$, calculated for all projected VLBI baselines that could be possibly observed with Earth-based VLBI (i.e., for all baselines with $\sqrt{u^{2}+v^{2}}$ less than the diameter of the Earth), separating the sources into four classes as follows:

$$
\text { Structure Index }=\left\{\begin{array}{l}
1, \text { if } 0 \mathrm{ps} \leq \tau_{\text {median }}<3 \mathrm{ps} \\
2, \text { if } 3 \mathrm{ps} \leq \tau_{\text {median }}<10 \mathrm{ps} \\
3, \text { if } 10 \mathrm{ps} \leq \tau_{\text {median }}<30 \mathrm{ps} \\
4, \text { if } 30 \mathrm{ps} \leq \tau_{\text {median }}<\infty
\end{array}\right.
$$

Based on this definition, the structure index at each of the three bands (X, K, and Q) was obtained for each source/session, thus providing an indication of the magnitude of the effects of intrinsic source structure in the session of observation. Detailed information regarding the computation of the structure delay corrections and the source structure index may be found in Charlot (1990) and Fey \& Charlot (1997). False color images of the structure corrections as a function of $(u, v)$ distance are available for each source via the Bordeaux VLBI Im- 
age Database ${ }^{13}$

From the source CLEAN components models, structure indices for 165 images of 138 sources imaged at $\mathrm{X}$ band, 1072 images of 274 sources at $\mathrm{K}$ band, and 267 images of 132 sources at $\mathrm{Q}$ band have been determined in a manner similar to that presented in Fey \& Charlot (1997, 2000). For each source that was successfully observed, Table 4 lists the number of sessions imaged along with the minimum and maximum values of the source structure index over the course of those sessions at each of the three frequency bands. In most of the cases, the difference between the maximum and minimum $S I$ is either 0 or 1 for $\mathrm{X}, \mathrm{K}$ and $\mathrm{Q}$ bands. For a few sources at $\mathrm{K}$ and $\mathrm{Q}$, the difference is 2, while there are no sources with a difference of 3 . Although the data are limited in number of sessions, this seems to indicate that the $S I$ is relatively stable over time.

As reported in $\$ 3$, average image dynamic ranges were $\sim 340: 1$ and $\sim 250: 1$ for $\mathrm{K}$ and $\mathrm{Q}$ band, respectively. The effects of dynamic range on $S I$ were investigated by varying the number of low-level image CLEAN components used to determine the median structure corrections and the structure index classification. Variation of the CLEAN components resulted in changes in the median structure correction on the order of a few tenths of a picosecond ( $0.7 \mathrm{ps} \mathrm{rms}$ ), with changes larger than $1 \mathrm{ps}$ occurring for only about $5 \%$ of the 1073 images at $\mathrm{K}$ band. These changes in the median structure correction resulted in a change in $S I$ by 1 unit for roughly $4 \%$ of the 1073 structure indices computed. Results for $\mathrm{Q}$ band were similar to those at $\mathrm{K}$ band. Thus, the structure indices determined in this study are not significantly affected by the dynamic range of the images.

Figures 7 and 8 compare the distributions of the source structure indices derived from our VLBA observations at the three bands, X, K, and Q. In Figure 7 we plot histograms of the maximum $S I$ measured for all sources in each of the three bands. The maximum is drawn from all available sessions in which the structure index for a particular source was determined. There were a total of 138 sources at X band, 274 sources at $\mathrm{K}$ band and 132 sources at $\mathrm{Q}$ band for which the $S I$ was measured. The figure shows a greater number of $S I=1,2$ (the most compact) sources at progressively higher frequencies. The percentage of $S I=1,2$ sources is 71,85 , and $92 \%$ for $\mathrm{X}-, \mathrm{K}-$, and Q-band, respectively. Figure 8 plots histograms of the maximum structure index measured for only the 97 sources common to all three frequencies in our VLBA observations. Again, the obvious trend toward lower structure index at higher frequencies is seen. For the $97 \mathrm{com}-$ mon sources, the percentage having $S I=1,2$ is $71 \%$ at $\mathrm{X}, 87 \%$ at $\mathrm{K}$, and $93 \%$ at $\mathrm{Q}$ band. These results indicate that the impact of source structure should be reduced at higher frequencies.

\subsection{Source Compactness}

The source structure index and its underlying quantity, the median structure delay correction, have proven to be highly useful measures of source structure and its effects on astrometric observations (Charlot et al. 2005; Fey et al. 2004, Sovers et al. 2002). In addition to these quantities, we introduce here a second indicator of structure

13 http://www.obs.u-bordeaux1.fr/BVID/ called the source compactness, $C$, described earlier in $\S 3$. The compactness, which is proportional to $S_{\text {beam }} / S_{\text {total }}$, provides an indication of how point-like or extended the radio emission from a particular source is on a continuous scale from 0.0 to 1.0 , where 1.0 represents a source with no extended emission.

The compactness was determined for all sources in all sessions for each of the three observing frequencies. Session-based compactness values were used to determine the average compactness over all sessions $(\bar{C})$ for each source. Shown in Figure 9 are the distributions for $\bar{C}$ for all sources imaged in the program including 138 sources at $\mathrm{X}$ band, 274 sources at $\mathrm{K}$ band, and 132 sources at $\mathrm{Q}$ band. The mean and median values of the distributions at $\mathrm{X}$ band are 0.83 and 0.86 , or greater than $80 \%$ of the maximum possible compactness $\bar{C}=1.0$. The mean and median values at $K$ band are slightly lower with values of 0.81 and 0.83 , respectively. For $\mathrm{Q}$ band, the results are nearly identical to those at $K$ band, with a value of 0.81 for both the mean and median of the distribution of $\bar{C}$. In Figure 10 the distributions of $\bar{C}$ are plotted for the 97 sources common to all three observing bands. The results here are very similar to those shown for all sources with mean (median) values of 0.84 (0.87), 0.79 (0.81), and $0.80(0.80)$ for the X-, K-, and Q-band distributions, respectively. While these results suggest only minor differences between the distributions of the mean source compactness at the three frequencies, one should recall that the compactness is dependent upon the flux density contained within one beam-width, $S_{\text {beam }}$, which, in turn, is dependent upon the frequency of the observations. Therefore, a constant value for the compactness with increasing frequency still implies a reduced intrinsic source size.

The source compactness also provides a convenient way to track changes in the source structure from one observing session to the next. To measure these changes, we computed the source compactness variability index $\left(\sigma_{C} / \bar{C}\right)$, which is simply the standard deviation of the compactness divided by its mean. A minimum value, $\sigma_{C} / \bar{C}=0.0$, corresponds to no variation in the compactness over time. Shown in Figure 11 are the distributions of source compactness variability index for sources imaged in more than one session at $\mathrm{K}$ and $\mathrm{Q}$ bands, respectively. The mean (median) compactness variability for the 235 sources at $\mathrm{K}$ band is 0.08 (0.06). For the 82 sources at $\mathrm{Q}$ band mean (median) values of $\sigma_{C} / \bar{C}$ are slightly smaller $0.07(0.05)$. These variations are small (no more than about $8 \%$ on average), however the probability that the compactness variability is significantly different from zero has not been determined. As in the case of the flux density variability, the compactness variability results are limited to the $\sim 5$ yr period over which our VLBA data were recorded.

\section{COMPARISONS AND DISCUSSION}

In the previous section, we discussed results for several quantities that emerged from our analysis of the VLBA images, namely: the source flux density, the source structure index, the source compactness, and the time variability of each of these quantities. In this section, we compare the source structure index to the compactness in order to verify that the two quantities are related. 
In addition, we compare both the structure index and the compactness to two astrometric quantities derived in Paper I, namely: the formal position uncertainties and position variability of the sources. These comparisons were made to determine whether the reduced structure effects seen at high frequency correspond to more precise astrometric positions.

\subsection{Structure Index and Source Compactness}

As an initial test of the source compactness, we compared $\bar{C}$ to the source structure index, $S I$, at each of the two frequencies. Shown in Figure 12 are the distributions of the mean source compactness for the 274 sources imaged at $\mathrm{K}$ band separated in terms of maximum source structure index. Figure 13 shows similar distributions for the 132 sources imaged at Q band. The three panels in each figure show $S I=1,2$ and 3 sources, respectively. There are two sources at $\mathrm{K}$ band and one source at Q band with $S I=4$ that are not shown in the figures. In both figures, it is evident that the distributions in compactness among the three structure indices are quite different with the distribution for $S I=1$ being strongly peaked at both frequencies, and the distributions broadening with increasing values of $S I$. Within each panel of Figures 12 and 13 the mean and median of the distribution are given. These values are also summarized in Table 5. From the table we see that for both frequencies, the mean and median source compactness is directly related to structure index. The correspondence between $S I$ and $\bar{C}$ is not unexpected, since both quantities provide an indication of the source structure as derived from the VLBI imaging.

We also compared the compactness variability index to the source structure index and the results are shown in Figure 14. Plotted in the figure is $\sigma_{C} / \bar{C}$ at $\mathrm{K}$ band as a function of each of the three structure indices, $S I=1$, 2 and 3 . Recall that the compactness variability index was determined for only those sources observed in more than one session (235 sources at $\mathrm{K}$ band and 82 sources at $\mathrm{Q}$ band). The equivalent plot for the $\mathrm{Q}$ band data was not produced because of the greatly reduced number of sources. As in the case of the source compactness, the variability in the compactness shows a clear trend with $S I$, with the $\sigma_{C} / \bar{C}$ distribution being the most narrow for $S I=1$ and the broadest for $S I=3$. The mean (median) values for each distribution are 0.04 (0.04), 0.08 $(0.07)$ and $0.15(0.14)$ for the $S I=1,2$ and 3 sources, respectively. A variability index $\sigma_{C} / \bar{C}=0$ indicates no variability in the source compactness from one session to the next. This relationship between the compactness variability index and the structure index suggests that the sources with the most structure as measured by $S I$ exhibit increased variability in their structure as measured by $\sigma_{C} / \bar{C}$.

\subsection{Structure Index, Compactness and Source Position Uncertainty}

The impact of source structure on the high-frequency CRF can be further studied by comparing structure index with the formal precision of the source positions comprising a potential CRF. The formal position uncertainties were taken from the K-band astrometric solution detailed in Paper I. For this solution, we used the CALC/SOLVE software package maintained by the NASA Goddard Space Flight Center (GSFC) to perform a least-squares astrometric solution for the K-band data. The 10 diurnal K-band experiments encompassed 82,334 measurements of bandwidth synthesis (group) delay and phase delay rate. Geodetic parameters estimated for each session include: station positions, 20-minute piecewise linear continuous troposphere zenith parameters, tropospheric gradients in the east-west and north-south directions, linear in time, estimated once per $24 \mathrm{hr}$ session, quadratic clock polynomials to model the gross clock behavior, and 60-minute piecewise linear continuous clock parameters. Corrections for ionospheric refraction drawn from Global Positioning System (GPS) total electron content (TEC) maps were applied to K-band data as discussed in Paper I. Positions for sources having three or more measurements of group delay were the only global parameters that were estimated.

The K-band catalog derived from the astrometric solution is comprised of positions and associated formal uncertainties for 268 sources. Because there were too few sources at Q-band (131) to separate into the four structure index categories, we chose only to compare the K-band uncertainties with structure index and compactness. An initial comparison of all 268 sources showed the position uncertainties plotted as a function of $S I$ category to be sensitive to a few outliers with relatively few observations. We, therefore, decided to restrict the comparison to sources with 100 or more group delay measurements. This same restriction was used by Fey \& Charlot (2000) in a similar study performed at X band.

Shown in Figure 15 are the distributions of the formal position uncertainties in a) $\alpha \cos \delta$ and b) $\delta$ for 193 sources with 100 or more group delay measurements at $\mathrm{K}$ band. The distributions are separated into the three $S I$ categories 1,2 , and 3 . There were two $S I=4$ sources with greater than 100 delay measurements that are not shown. There is good agreement between the mean and median values suggesting little or no dependence on outliers. The results show no significant difference in the mean and median position uncertainties from one structure index category to the next. In addition, we find the mean and median position uncertainties in $\delta$ are roughly twice the uncertainties in $\alpha \cos \delta$ for all three $S I$ categories.

In Fey \& Charlot (2000), it was shown that the mean and median X-band source position uncertainties in both $\alpha \cos \delta$ and $\delta$ increased regularly as a function of structure index from $S I=1$ to 4 . In addition to the 100 group delay measurement restriction, Fey \& Charlot (2000) used the formal position uncertainties from $\overline{M a}$ et al. (1998), which were adjusted by the standard ICRF inflation factor described therein. In order to accurately compare our K-band values, we applied the same inflation factor (the root sum square (rss) of 1.5 times the formal uncertainty and 0.25 mas) to the K-band formal uncertainties. Table 6 lists the mean and median position uncertainties at both X and $\mathrm{K}$ bands as a function of $S I$ category. Again we see that there is no increase in position uncertainty from structure index 1 to 3 at K-band unlike the earlier findings at X-band. More importantly, the table shows that for a similar number of sources, the inflated mean/median position uncertainties are smaller at $\mathrm{K}$ band than at $\mathrm{X}$ band for all structure index categories. 
It should be noted, that there have been significant improvements in both the number and quality of the VLBI observations since the construction of the ICRF, and the Fey \& Charlot (2000) values reported in Table 6 do not reflect the accuracy of the current X-band CRF.

In addition to the comparison of the $S I$ and the formal uncertainties, we compared the mean source compactness $(\bar{C})$ to the formal uncertainties. Recall that the maximum possible value of the source compactness $C=1.0$ indicates that all of the source flux density is contained within one synthesized beam. The mean compactness is just an average over all of the sessions in which the source was imaged. Figure 16 shows the formal uncertainties in the position versus the source compactness. The plotted formal position uncertainty is the rss of the uncertainties in $\alpha \cos \delta$ and $\delta$. The figure also shows the structure index for each source by point color and type, with green circles, blue triangles, and red squares indicating maximum $S I$ values of 1,2 , and 3 respectively. Figure 16 does not show a clear trend in the formal position uncertainty versus compactness. This is to be expected since it was previously shown that the $\bar{C}$ and $S I$ are correlated, and no significant relationship was found for the position uncertainty as a function of $S I$ (e.g. Figure 15).

\subsection{Structure Index, Compactness, and Source Position Stability}

Another useful comparison can be made between the stability of the source positions over time and the source structure as traced by either the structure index or the source compactness. Time variation of the astrometric coordinates of $\mathrm{CRF}$ sources has previously been attributed to variability in the intrinsic source structure (c.f. Charlot 1994, 2002; Fey et al. 1997; Fey \& Charlot 2000). Thus, it is natural to search for any relationship between such variations and the structure index at higher frequencies. A measure of source position stability is the weighted root-mean-square (wrms) position variation. These variations were obtained from a series of Kband astrometric solutions described in Paper I. In these solutions, a fraction of the sources were treated as local parameters (i.e. an estimate of the position was derived for each session in which the source was observed) with the remaining sources treated as global. The estimation of source position stability was limited to 88 sources that were observed in five or more of the 10 VLBA sessions. Five was considered a sufficient number of sessions (position estimates) per source to derive reliable statistics.

From these solutions, a time series of source positions was generated for each source, and from these time series the wrms position variations were computed. Shown in Figure 17 are the distributions of the wrms position variations in $\alpha \cos \delta$ (a) and $\delta(\mathrm{b})$, respectively. The figure also lists the mean and median position variations for each distribution. In $\alpha \cos \delta$, the mean and median of the distribution are 0.14 and 0.12 mas, respectively. For $\delta$ the mean and median are roughly twice as large at 0.30 and 0.25 mas, respectively. As stated in Paper I, the larger wrms position variations in $\delta$ are likely due to the combination of ionospheric/tropospheric effects and network geometry, specifically the lack of long north-south baselines for the VLBA.

It would have been desirable to separate the wrms position variations into structure index categories, and to plot the distributions as a function of $S I$ as was done in 4.2 Because the structure index is quantized into four categories, there were simply too few sources observed in five or more sessions to determine reliable statistics for the separate $S I$ categories. Instead, in Figure 18, we plot the wrms variation against the mean source compactness previously described in $\$ 3.3$. The wrms variation plotted is the rss of the variations in $\alpha \cos \delta$ and $\delta$. In addition, the structure index for each source is shown by point type with green circles, blue triangles, and red squares indicating maximum $S I$ of 1,2 , and 3 respectively. Figure 18 does not suggest a clear trend in the compactness and wrms position stability. While it does appear that there are a number of sources clustered in a region of high compactness and low wrms variations, there are also sources that are less compact with equally low wrms variations. A more quantitative comparison between the $S I$ and the position stability will have to wait for additional highfrequency observations.

\section{SUMMARY}

We have produced a combined total of $1339 \mathrm{~K}$ - and Q-band images of 274 extragalactic sources from 10 sessions of VLBA data observed as part of a program to select high-frequency sources for use in spacecraft navigation and future CRFs. A detailed analysis of the images has allowed us to determine several quantities that can serve as useful indicators of both detectability and astrometric quality including: source flux density, flux density variability, structure index, source compactness, and compactness variability. The results of our imaging and analysis can be summarized as follows:

1. The sources described herein were selected from previous catalogs at lower (e.g. $8.4 \mathrm{GHz}$ ) frequencies. The distributions of source flux density at X, $\mathrm{K}$, and $\mathrm{Q}$ bands show mean values greater than $1 \mathrm{Jy}$ for each of the three bands. These results suggest a sufficient number of suitable sources for astrometric use at high frequencies.

2. For sources imaged at $24 \mathrm{GHz}$, we find that, on average, $1 \sigma$ variations in the session-to-session flux density were $20 \%-25 \%$ over the five-year period of our observations. Comparatively, variations of the flux density scale from one session to the next were less than $10 \%$. Variations in the source flux density are, thus, relatively small and it should be expected that sources will be observable from one session to the next.

3. There is a clear dependence of source structure index on observational frequency. The percentage of sources with $S I=1,2$ (the most compact) increases from $\sim 70 \%$ at $\mathrm{X}$ band, to $\sim 85 \%$ at $\mathrm{K}$ band, and to $\sim 92 \%$ at $\mathrm{Q}$ band. This suggests that the higher resolution afforded by the higher observing frequencies yields less structure at a fixed angular scale. This result is corroborated by the mean source compactness which shows that $80 \%$ of the total flux density is contained within the core at all three frequencies.

4. On average, the $1 \sigma$ variations in the source compactness at $\mathrm{K}$ and $\mathrm{Q}$-band were no more than about 
$8 \%$ at either frequency over the five-year period of our VLBA observations.

5. For the K-band data, we find a trend between the source compactness variability index (i.e., the variation of the compactness over time) and the source structure index indicating that sources with larger values of $S I$ exhibit increased variation in the structure as measured by $\sigma_{C} / \bar{C}$.

6. No clear trend was found between the formal uncertainties in the astrometric source positions and the source structure index at $\mathrm{K}$ band in contrast to the trend found at $\mathrm{X}$ band by Fey \& Charlot (2000).

The implications of the above results with regards to spacecraft navigational needs are twofold. First, there are numerous sources available at high frequencies, and these sources persist over relatively long (five years) periods of time. Second, the sources are more compact at these higher frequencies and should provide high-quality astrometric reference points for spacecraft navigation. Such sources also provide a foundation upon which to build a future CRF and are of benefit to radio astronomy, in general, as fiducial references. Future imaging observations should continue to improve our understanding of source structure at $\mathrm{K}$ and $\mathrm{Q}$ band and allow us to better characterize the variability of the structure over time and its effect on astrometric positional stability.

This research was partially supported through NASA contracts with the California Institute of Technology and the U.S. Naval Observatory. This research made use of the USNO Radio Reference Frame Image Database (RRFID).

\section{REFERENCES}

Blandford, R. D. \& Königl, A. 1979, ApJ, 232, 34

Charlot, P. 1990, AJ, 99, 1309

Charlot, P. 1994, in Proc. of Int. Symp.: VLBI Technology -

Progress and Future Observational Possibilities, Kyoto, Japan, 1993 September, ed. T. Sasao, S. Manabe, O. Kameya \& M. Inoue (Tokyo: Terra Scientific Publishing Company), 287

Charlot, P. 2002, in International VLBI Service for Geodesy and Astrometry: General Meeting Proc., 2002 May, ed. N. R. Vandenberg \& K. D. Baver, NASA/CP-2002-210002, 233

Charlot, P., Fey, A. L., Jacobs, C. S., Ma, C., Sovers, O. J., \& Baudry, A. 2005, Journées 2004-systèmes de référence spatio-temporels, 21

Clark, B. G. 1980, A\&A, 89, 37

Fey, A. L., \& Charlot, P. 1997, ApJS, 111, 95

Fey, A. L., \& Charlot, P. 2000, ApJS, 128, 17

Fey, A. L., Clegg, A. W., \& Fomalont, E. B. 1996, ApJS, 105, 299

Fey, A. L., Eubanks, T. M., \& Kingham, K. A. 1997, AJ, 114, 2284

Fey, A. L., et al. 2004, AJ, 127, 3587

Hovatta, T., Lehto, H. J., \& Tornikoski, M. 2008, A\&A, 488, 897
Hovatta, T., Tornikoski, M., Lainela, M., Lehto, H. J., Valtaoja, E., Torniainen, I., Aller, M. F., \& Aller, H. D. 2007, A\&A, 469, 899

Kovalev, Y. Y., Lobanov, A. P., Pushkarev, A. B., \& Zensus, J. A. 2008, A\&A, 483, 759

Lanyi, G. E., et al. 2009, AJ, submitted. (Paper I)

Lobanov, A. P. 1998, A\&A, 330, 79

Ma, C., et al. 1998, AJ, 116, 516

Napier, P. J., Bagri, D. S., Clark, B. G., Rogers, A. E. E., Romney, J. D., Thompson, A. R., \& Walker, R. C. 1994, Proceedings of the IEEE, 82, 658

Pearson, T. J., \& Readhead, A. C. S. 1984, ARA\&A, 22, 97

Pearson, T. J., Shepherd, M. C., Taylor, G. B., \& Myers, S. T. 1994, BAAS, 26, 1318

Rogers, A. E. E. 1970, Radio Science, 5, 1239

Sovers, O. J., Charlot, P., Fey, A. L., \& Gordon, D. 2002, in International VLBI Service for Geodesy and Astrometry: General Meeting Proc., 2002 May, ed. N. R. Vandenberg \& K. D. Baver, NASA/CP-2002-210002, 243

Teräsranta, H., Wiren, S., Koivisto, P., Saarinen, V., \& Hovatta, T. 2005, A\&A, 440, 409 


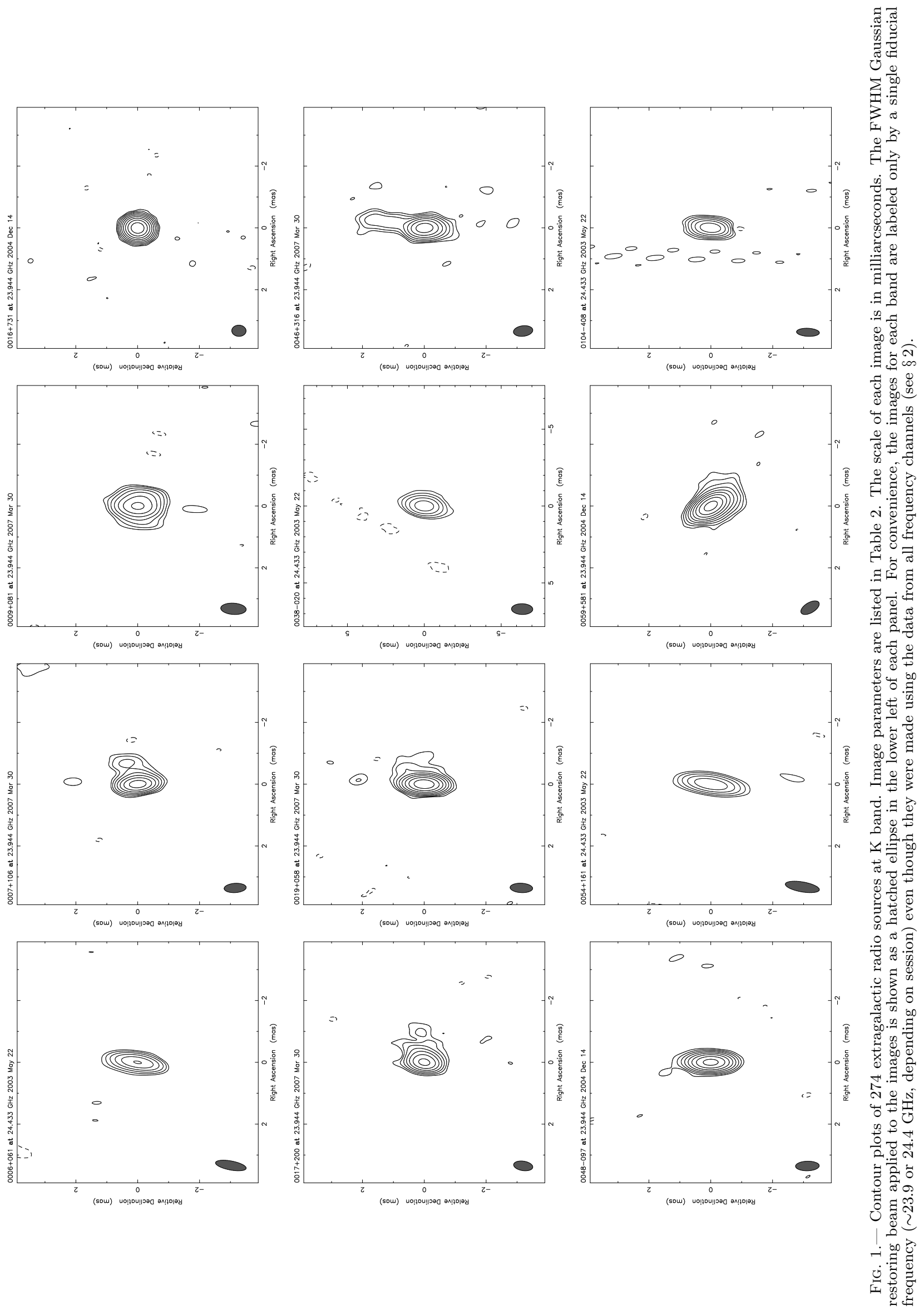



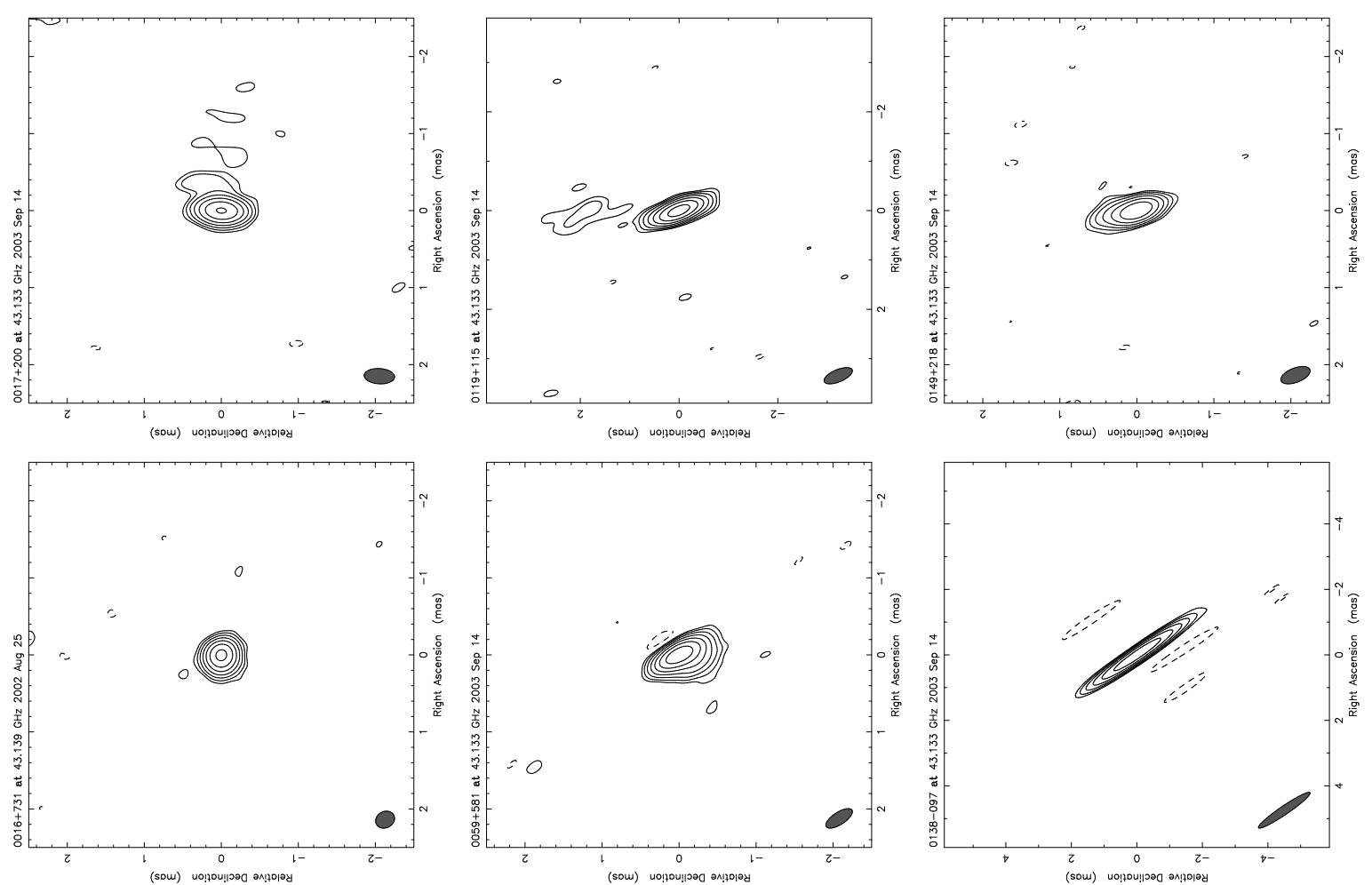

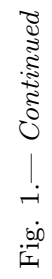
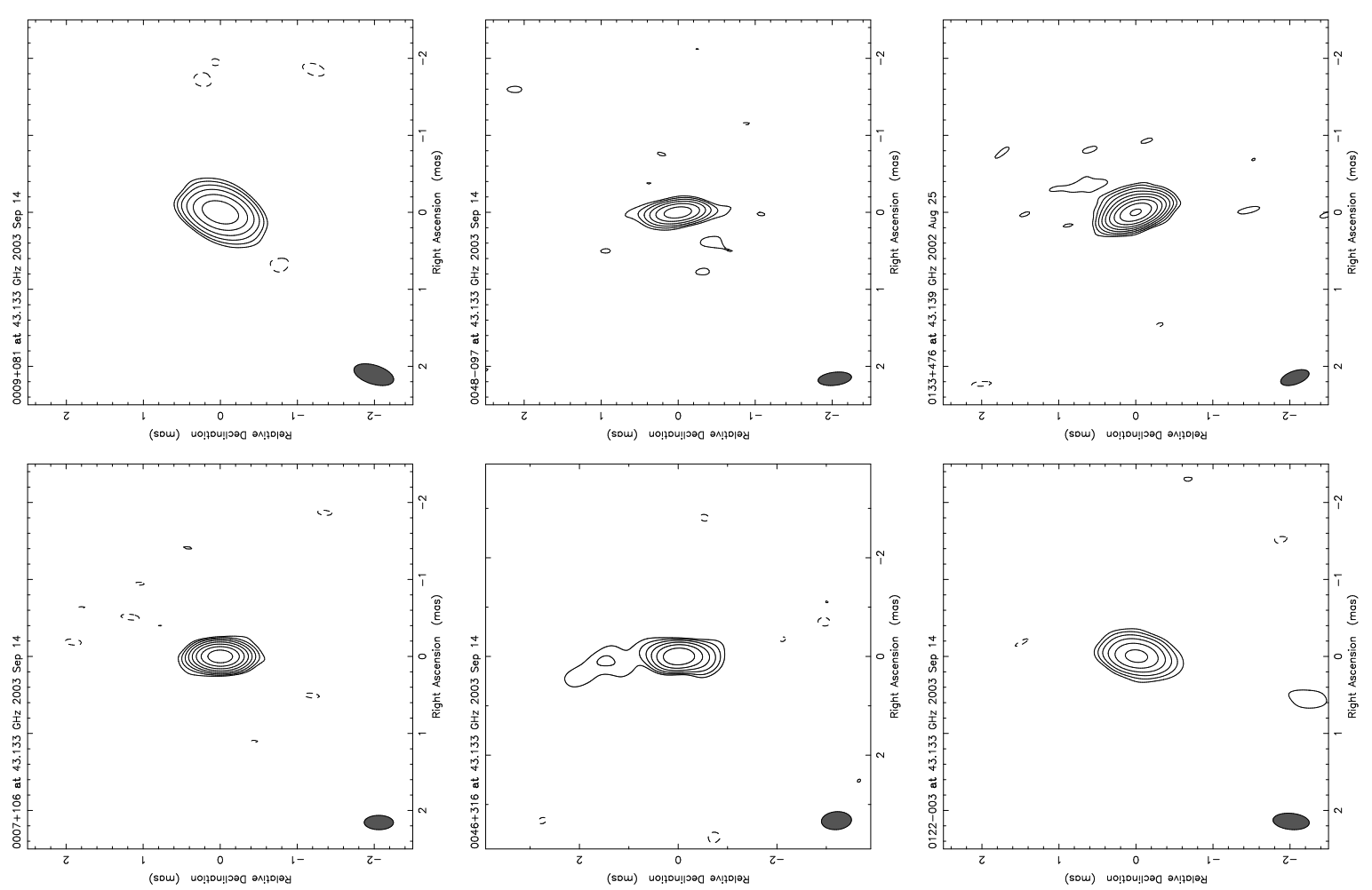

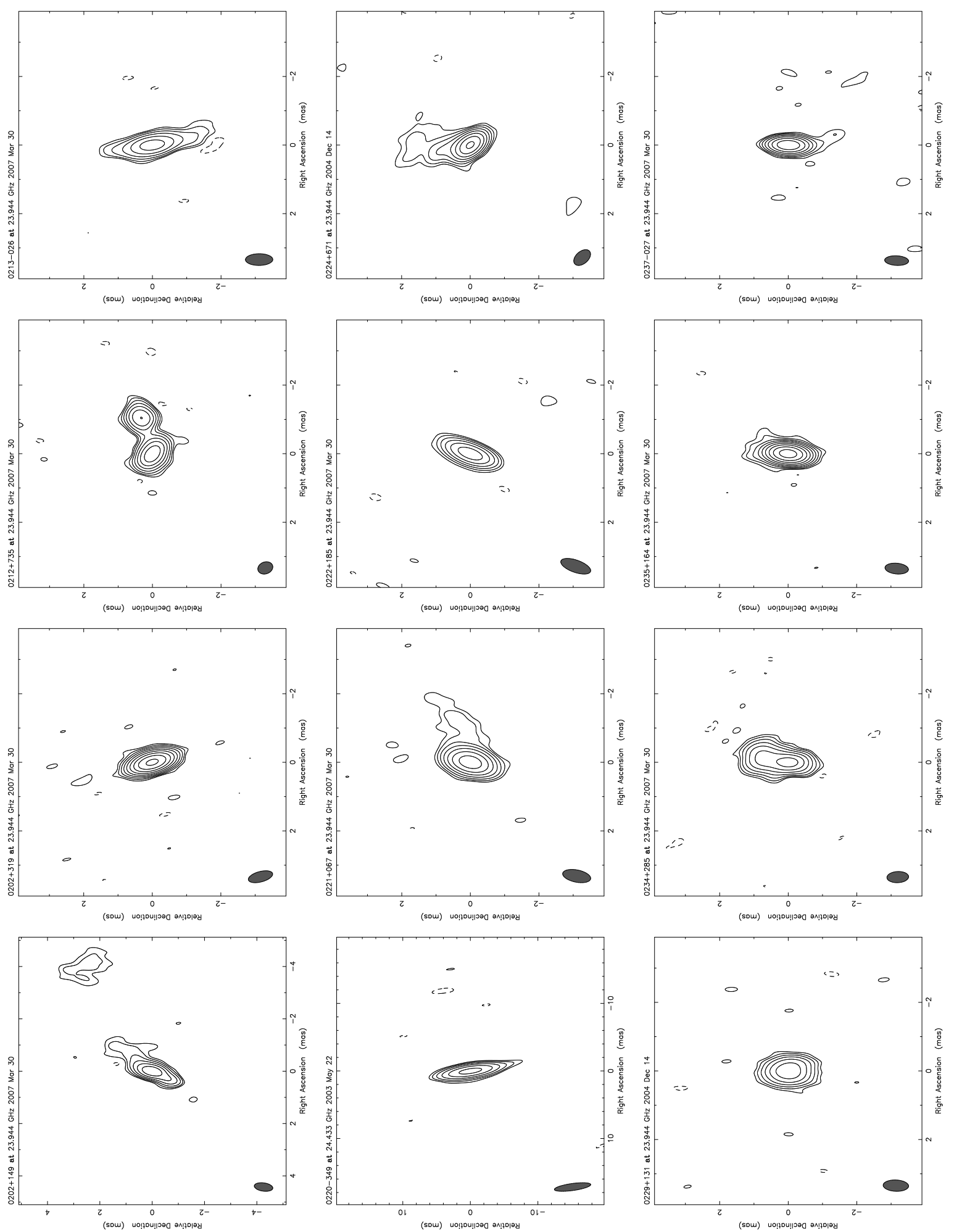

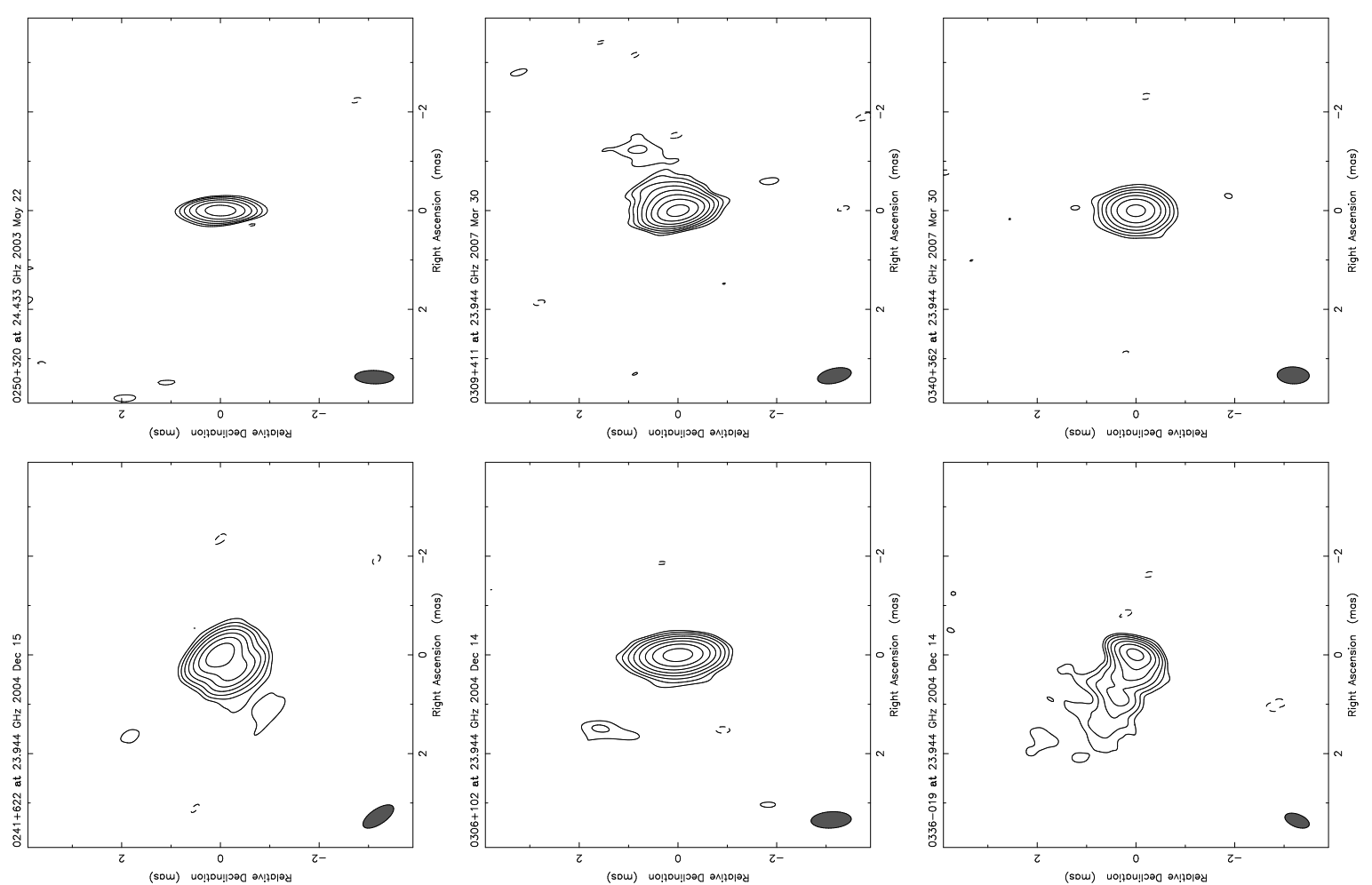

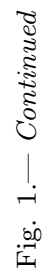
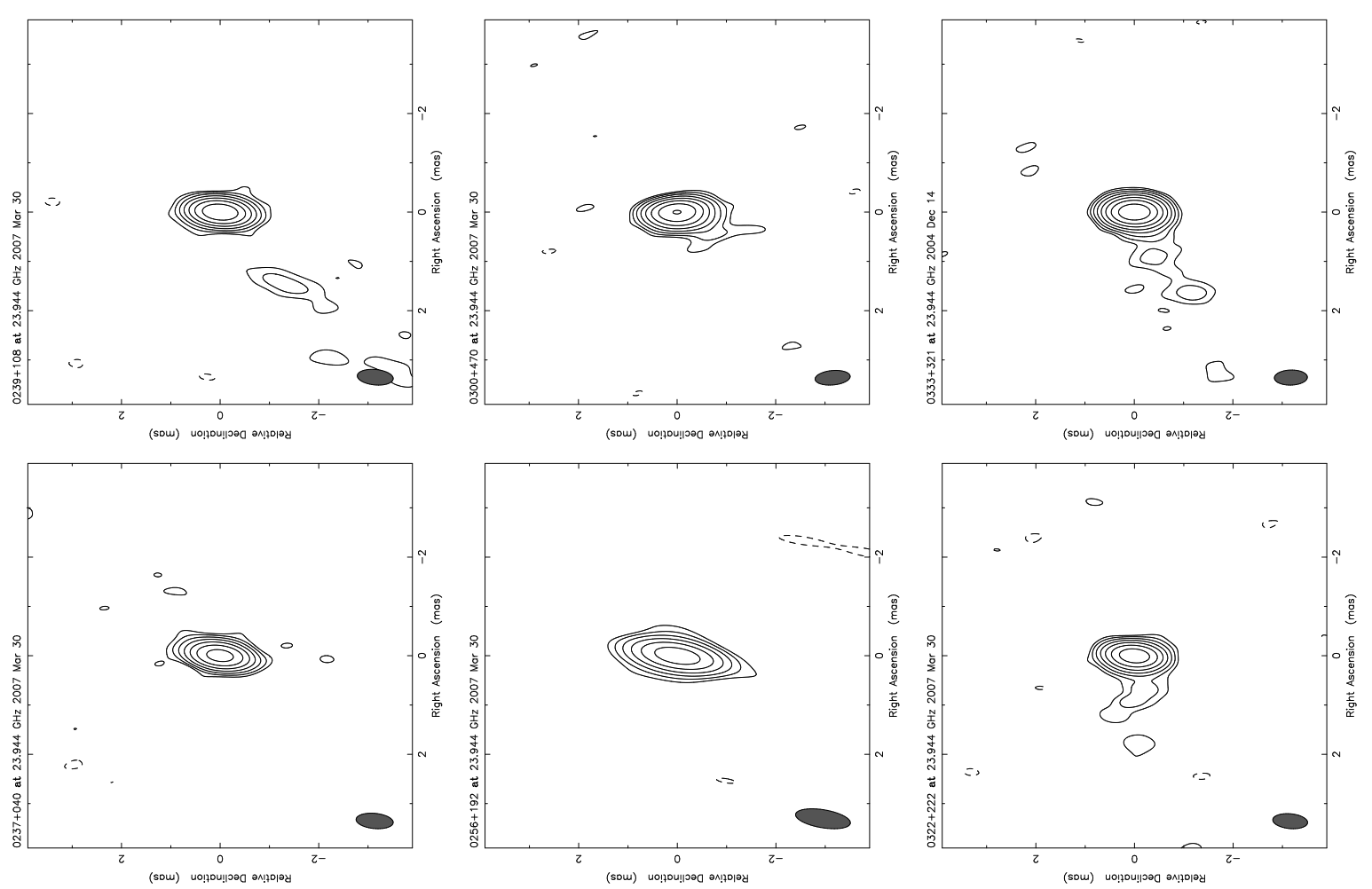

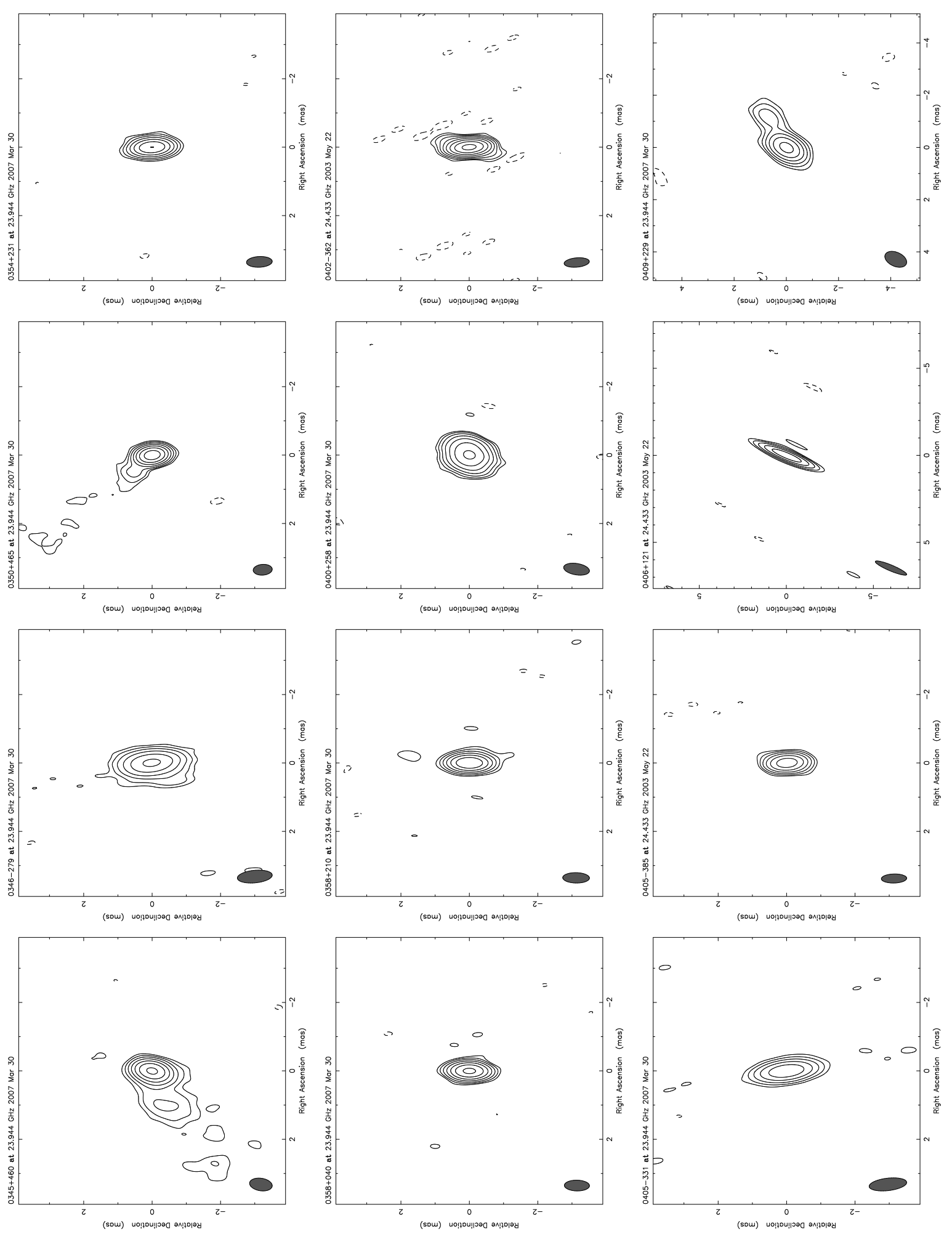

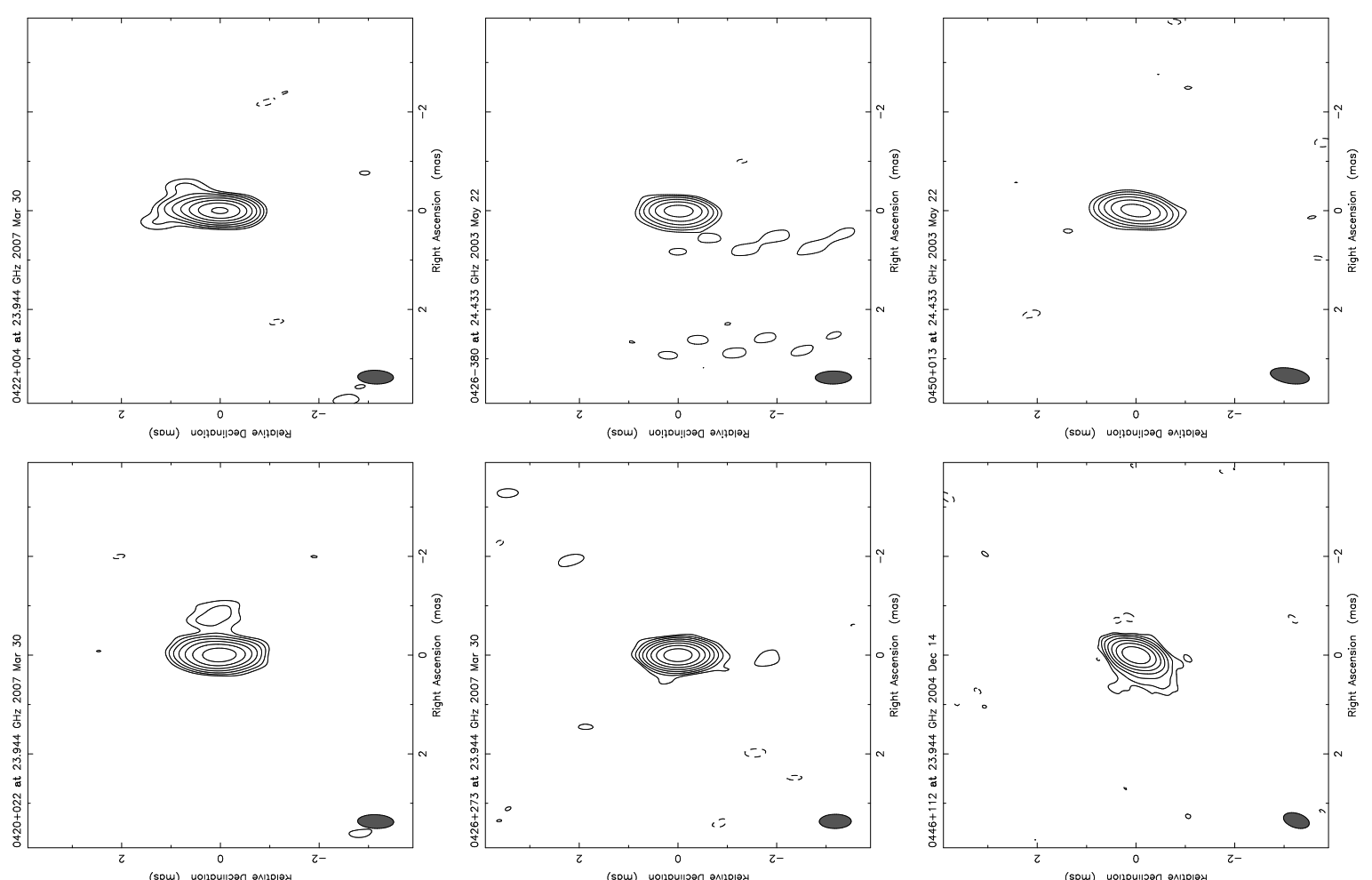

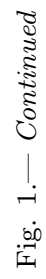
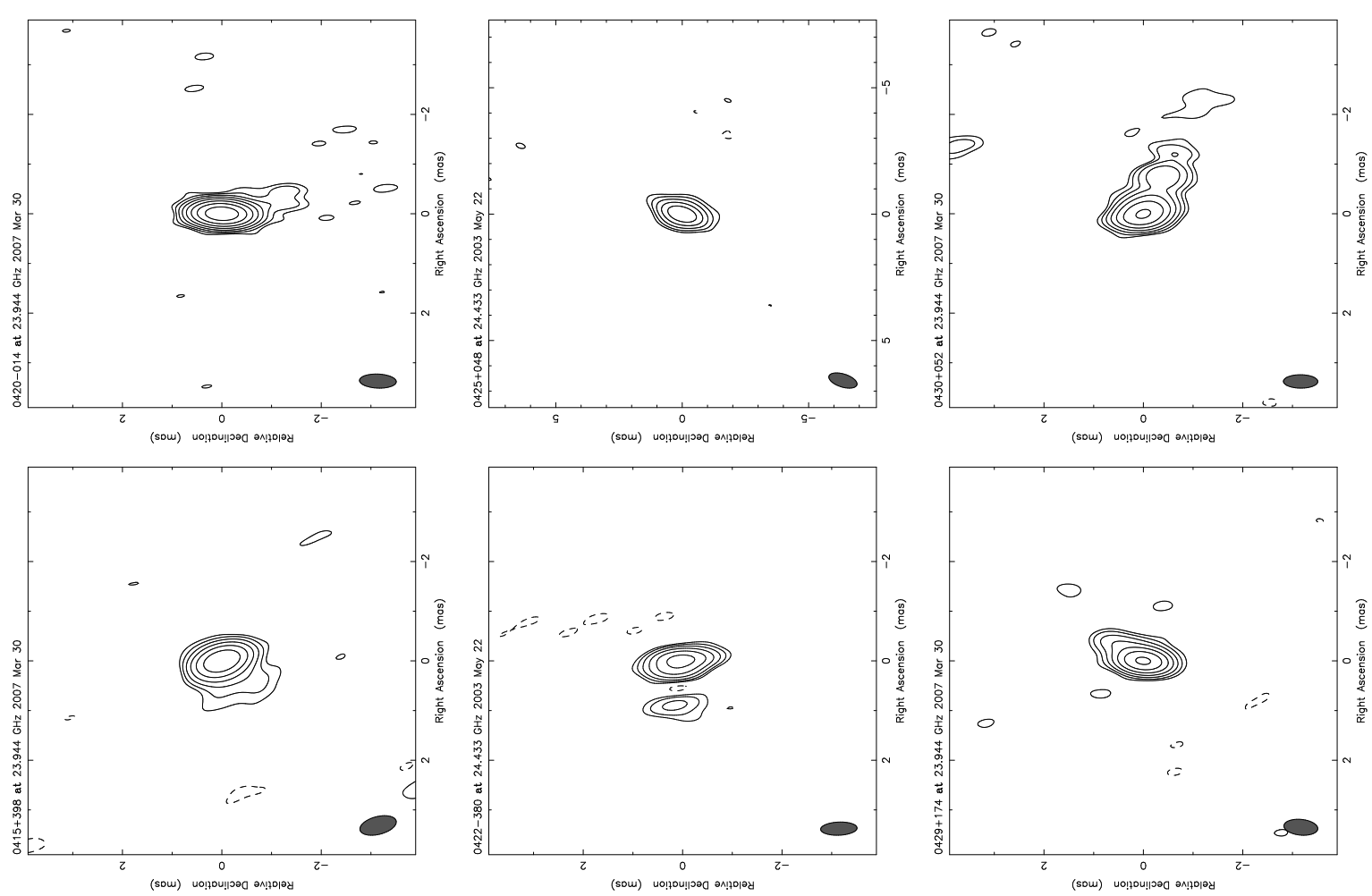

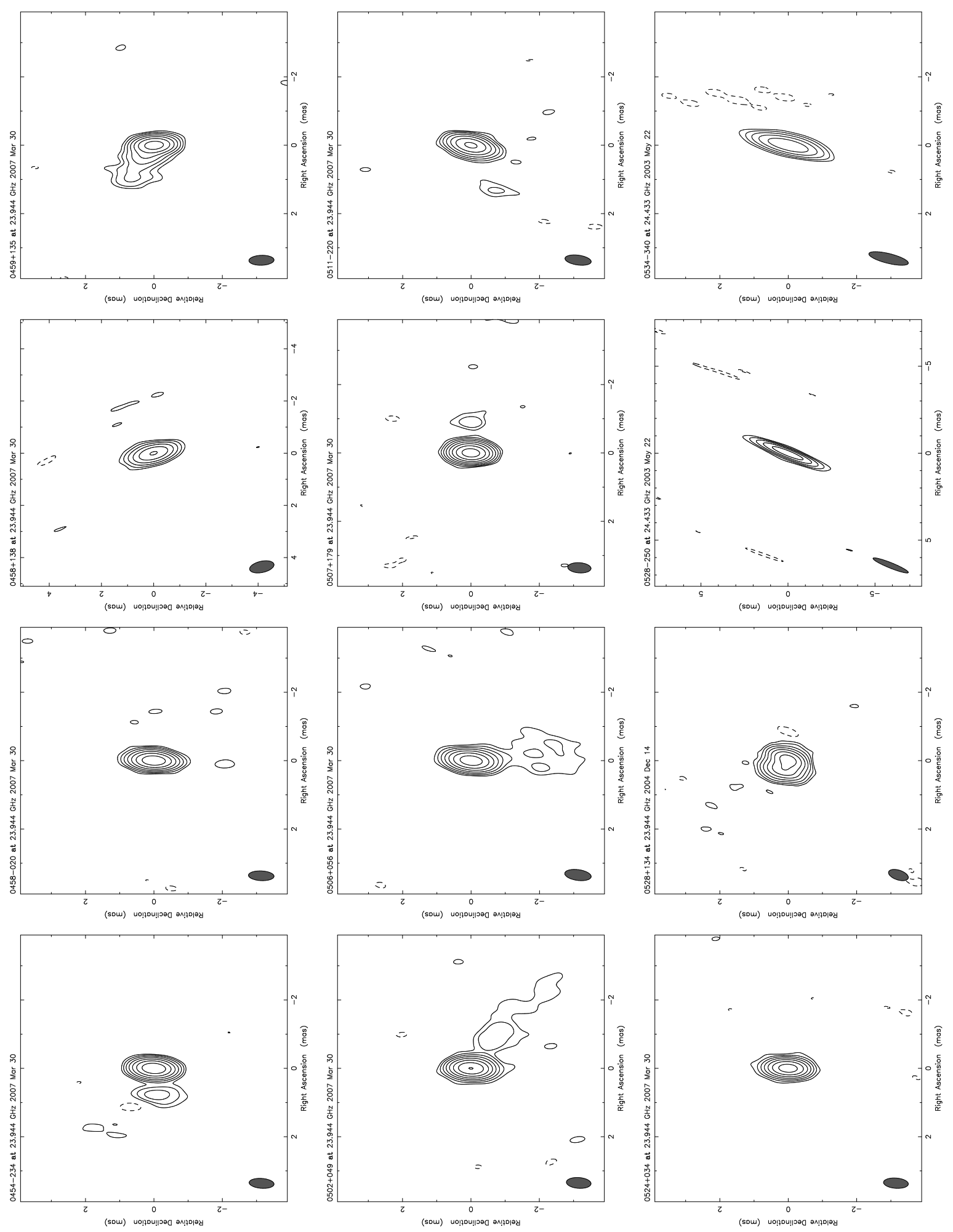

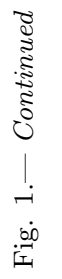



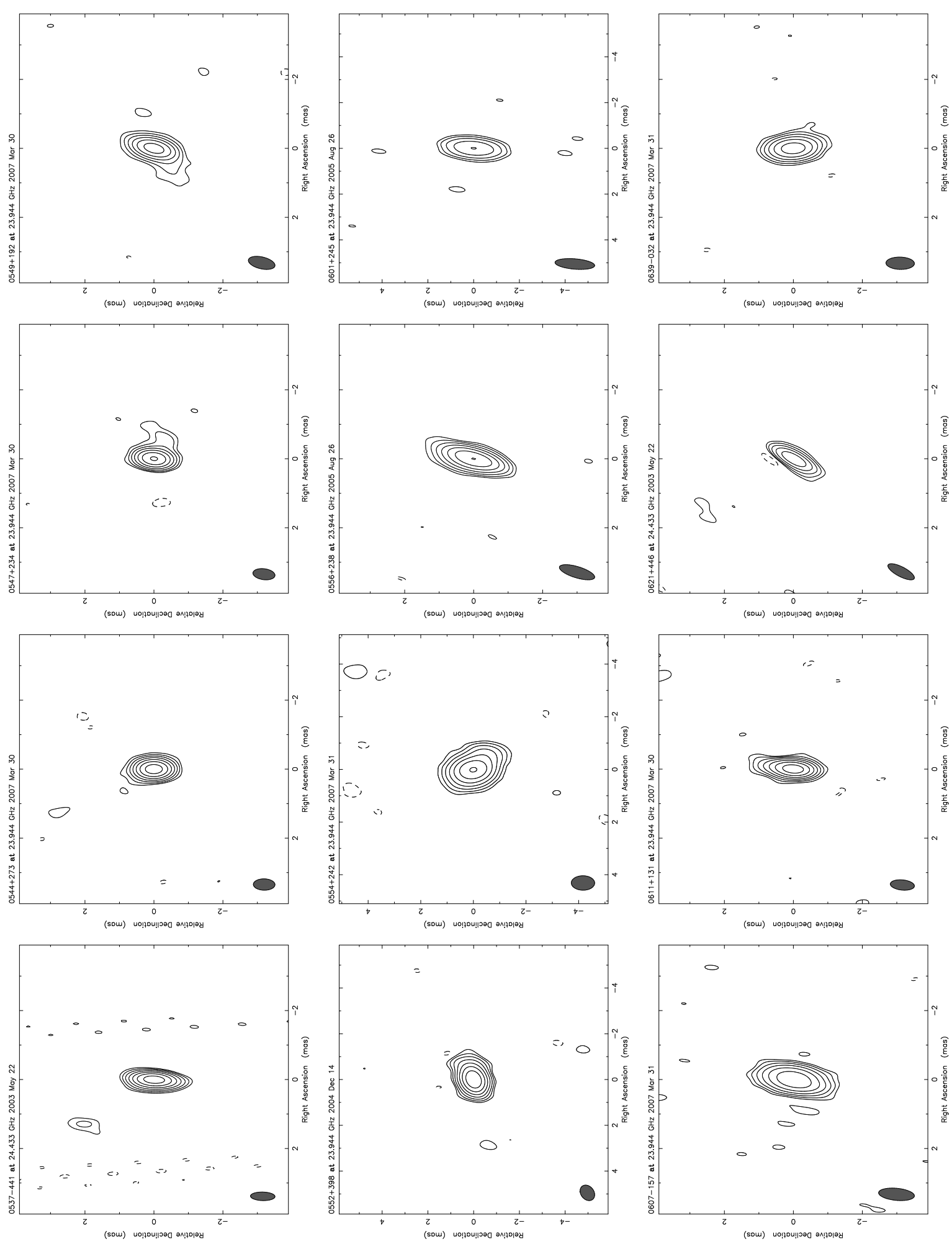

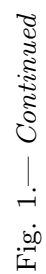



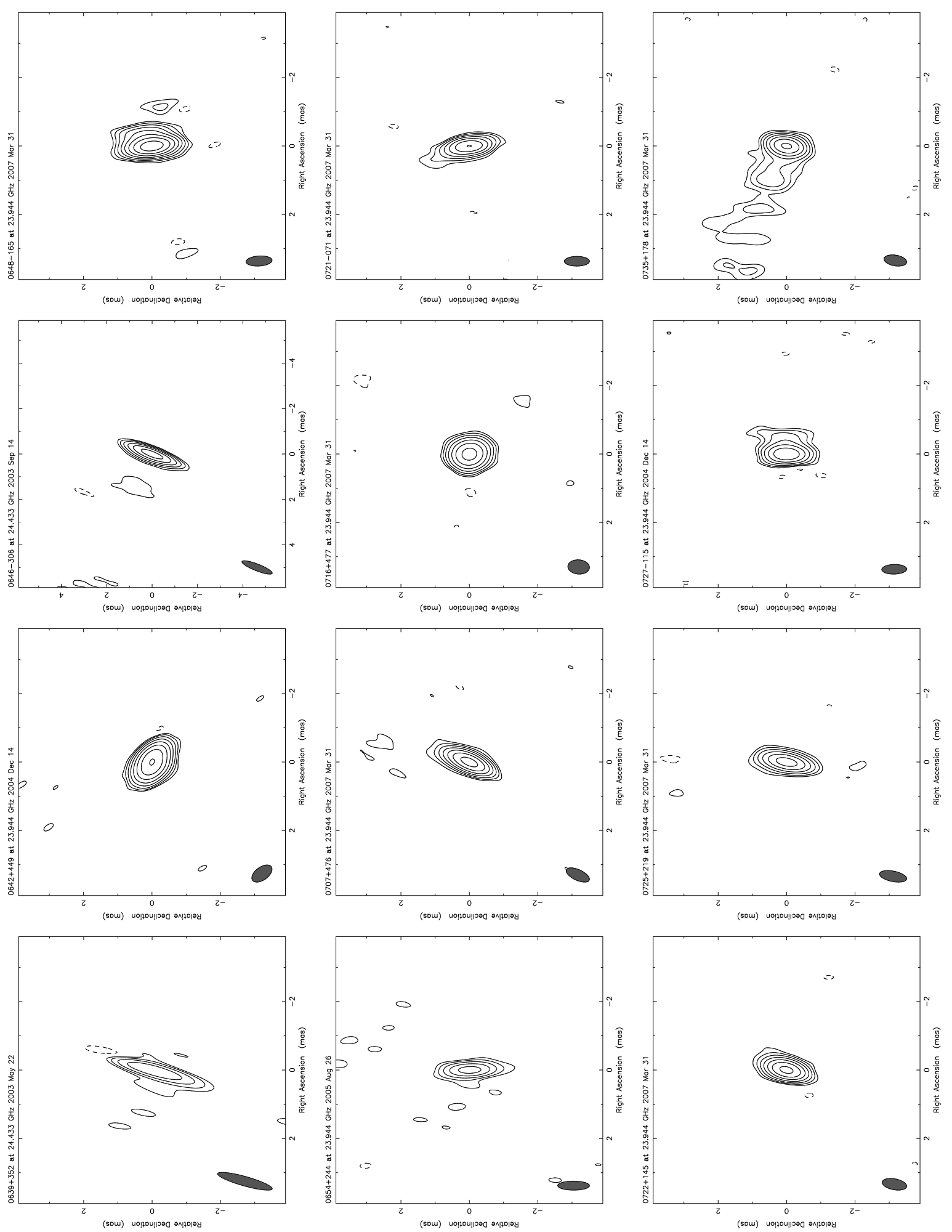

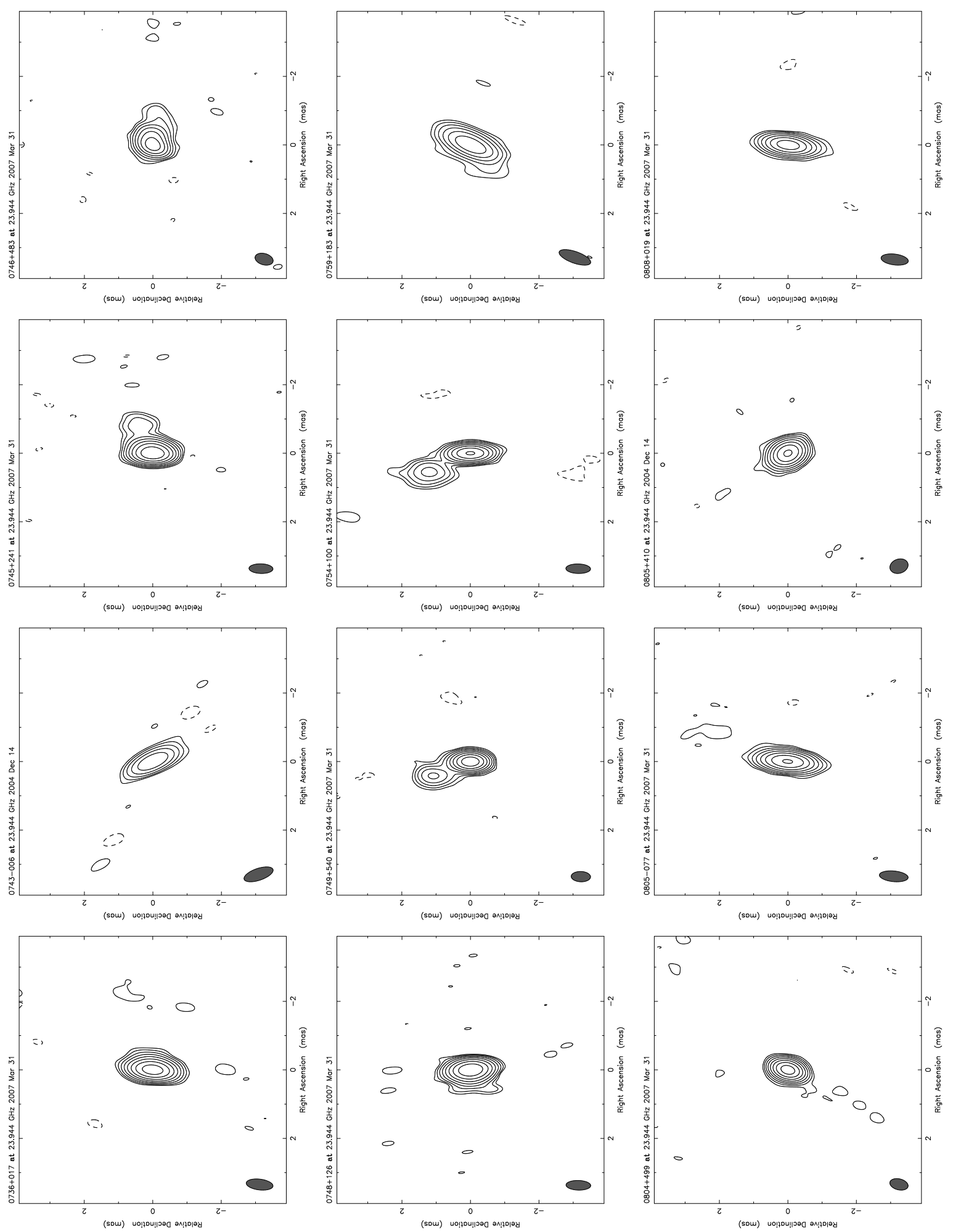

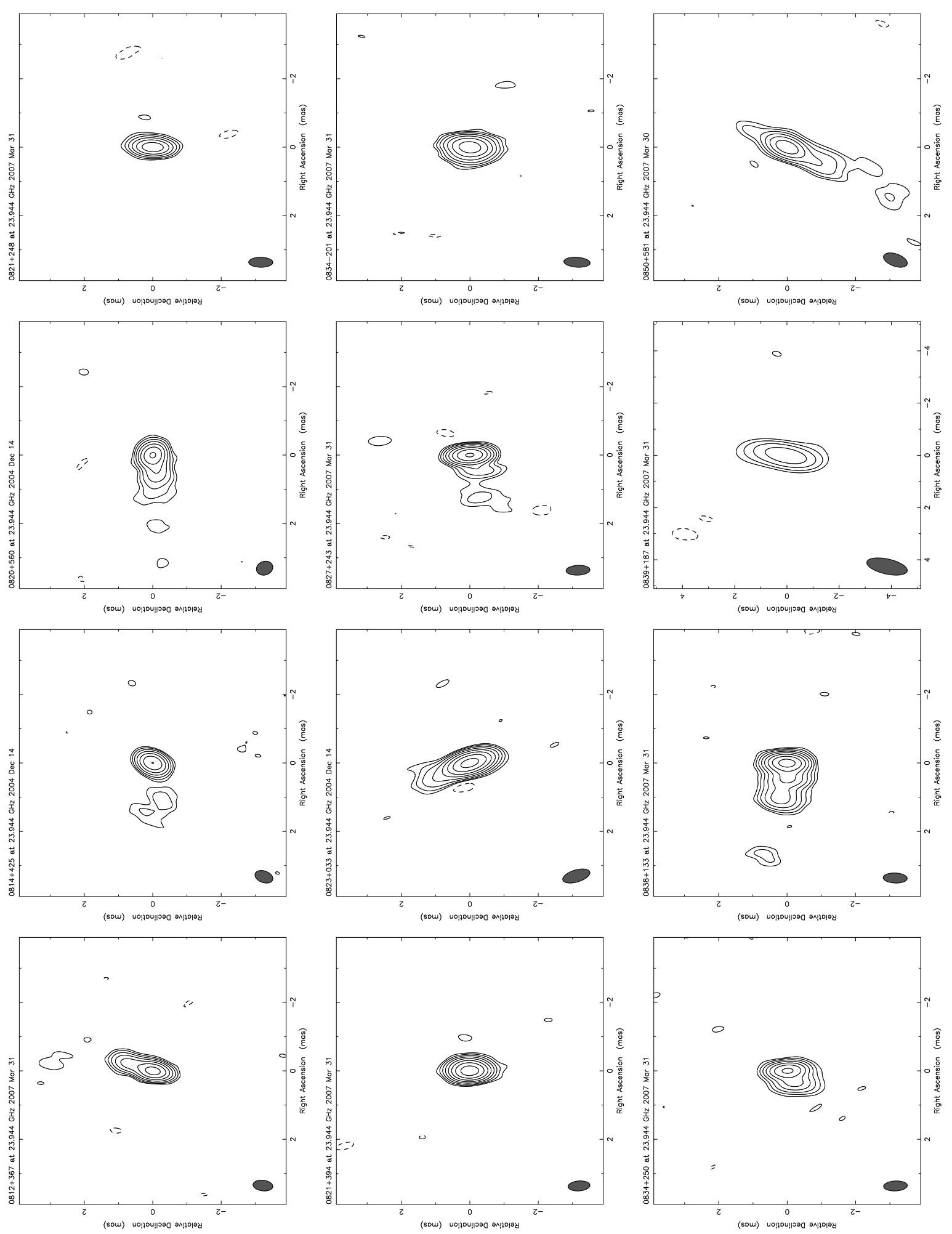
20

Charlot et al.
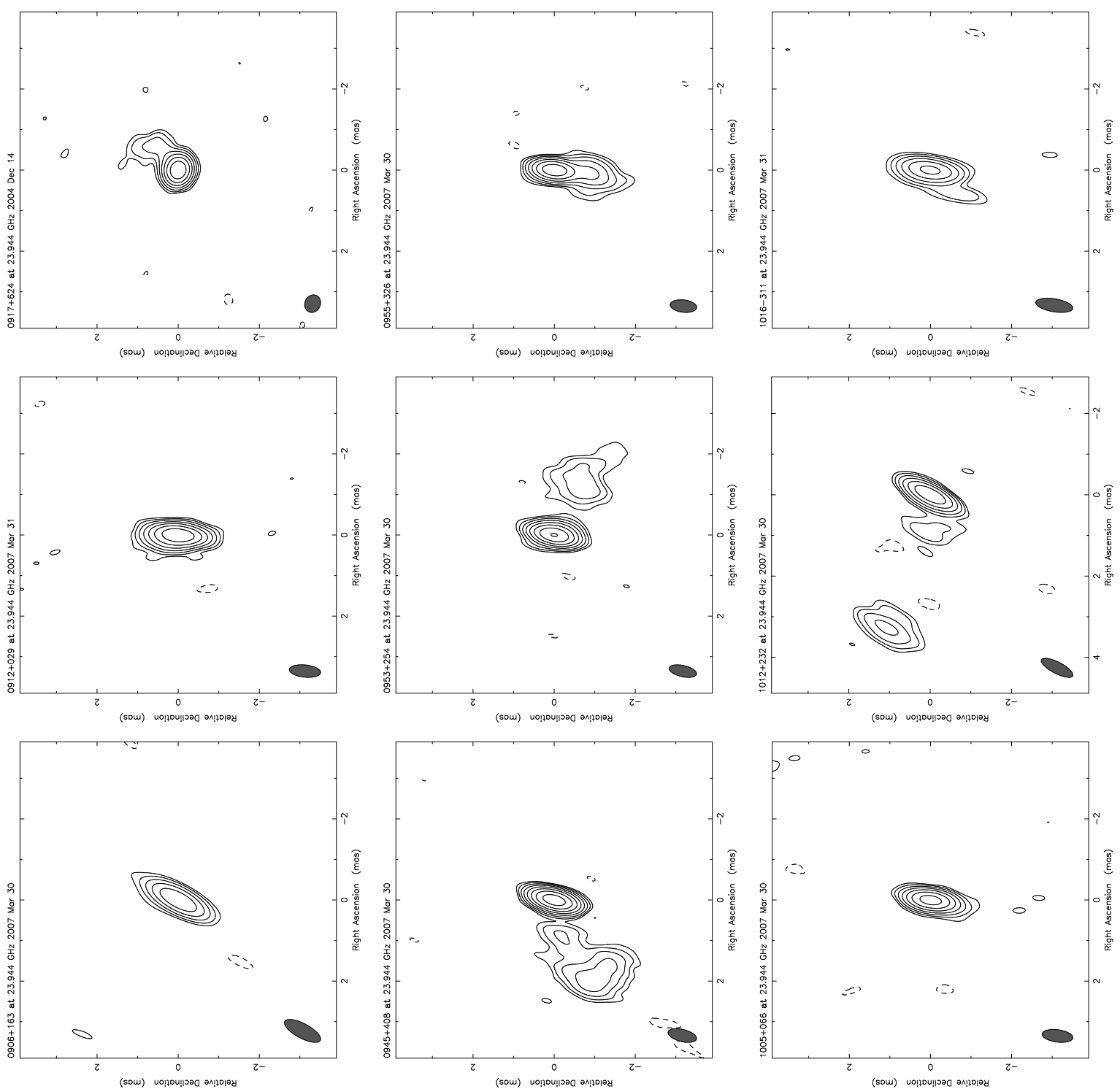

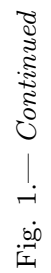
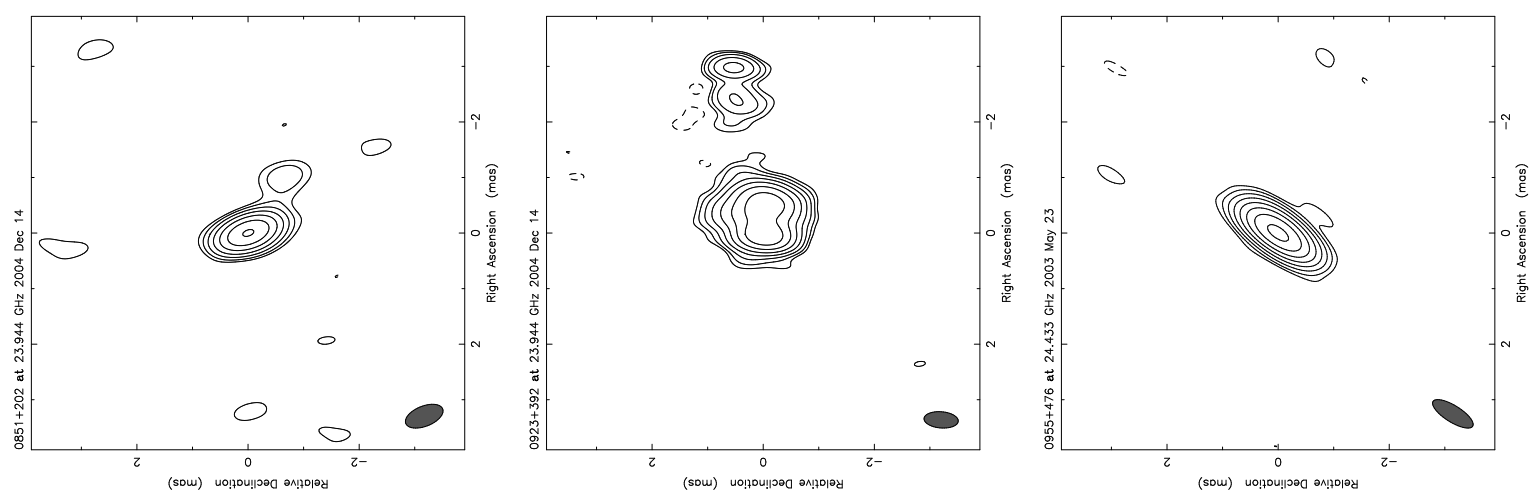

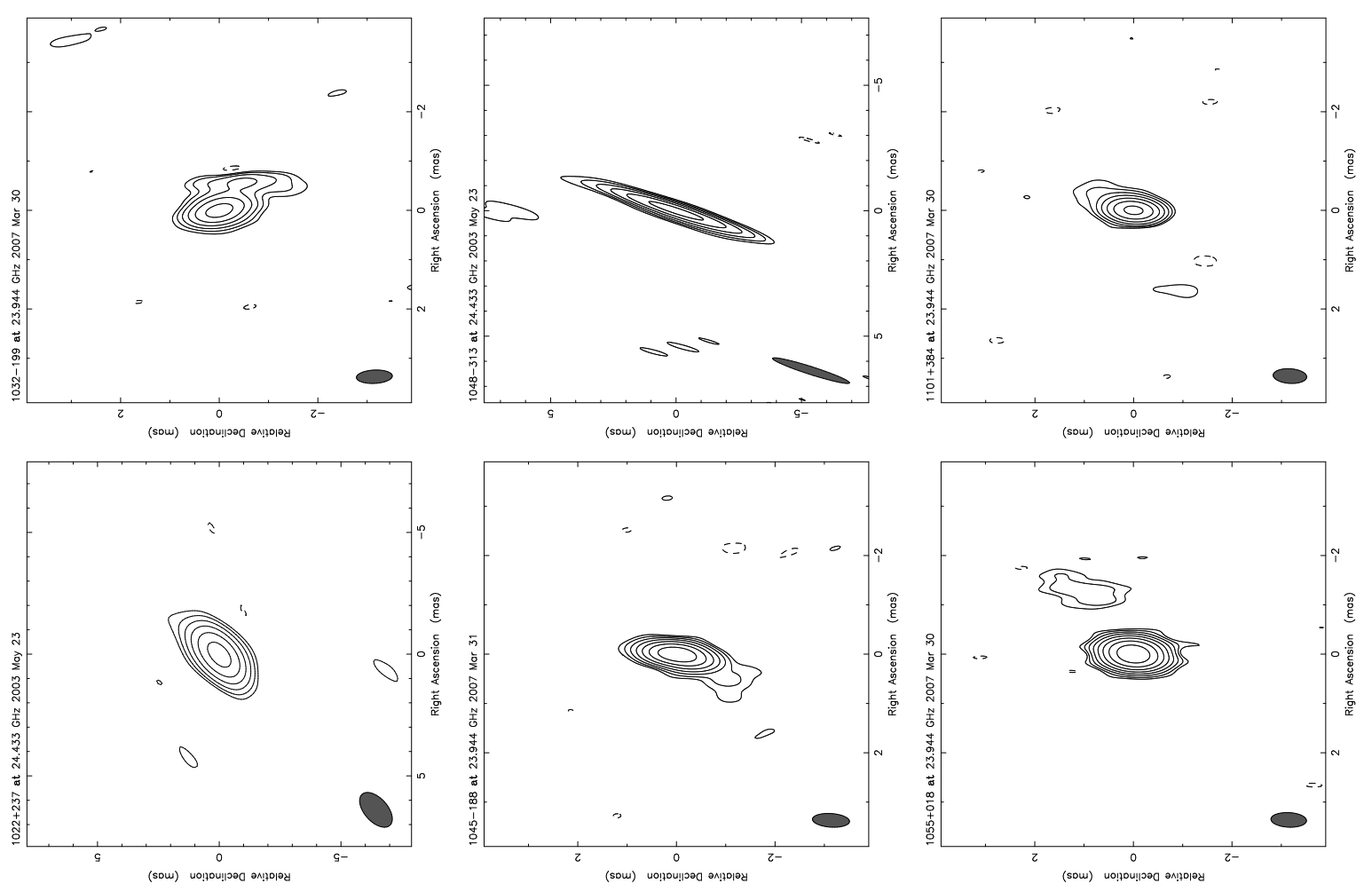

نे
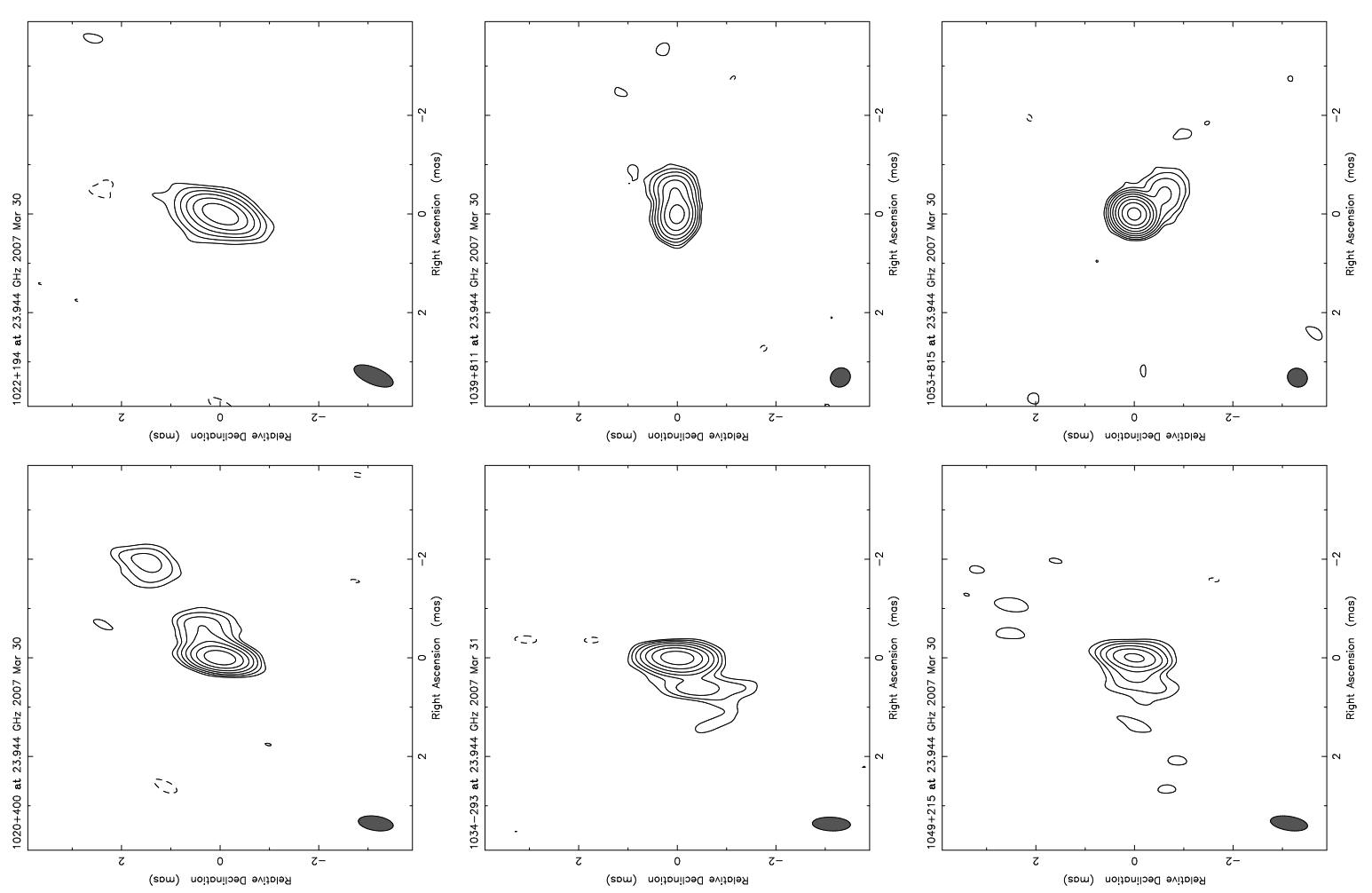

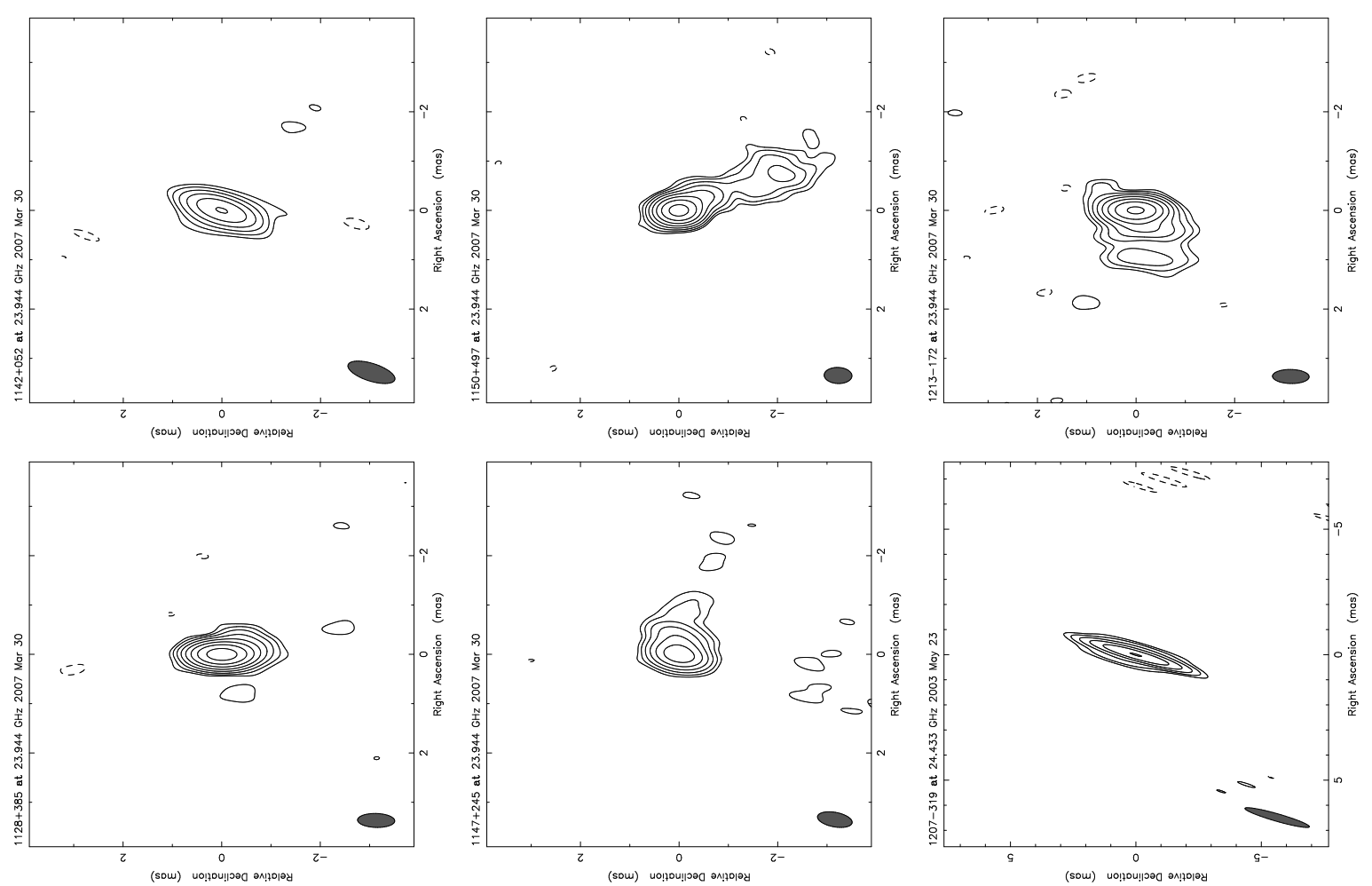

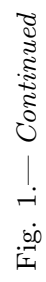
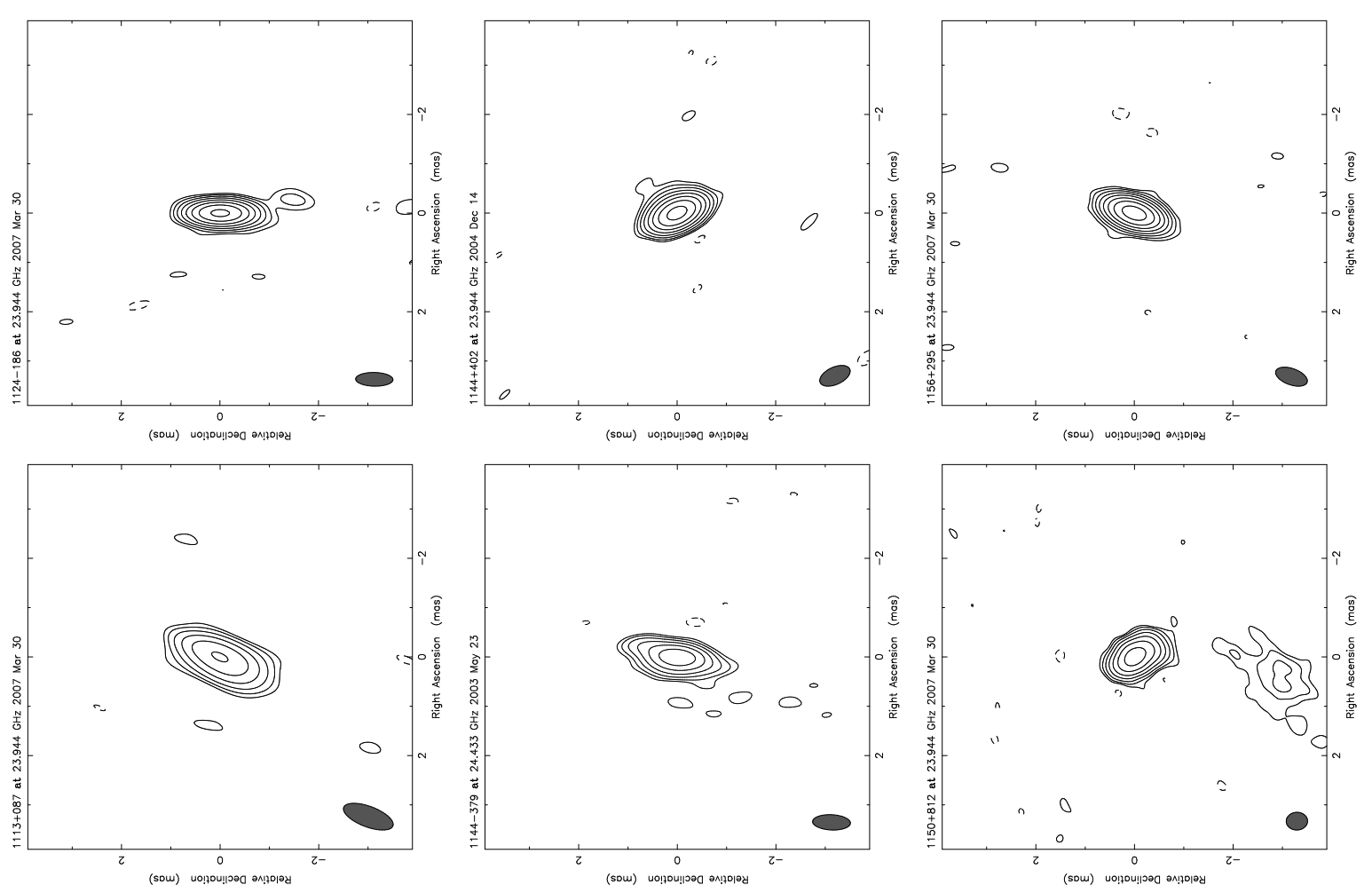

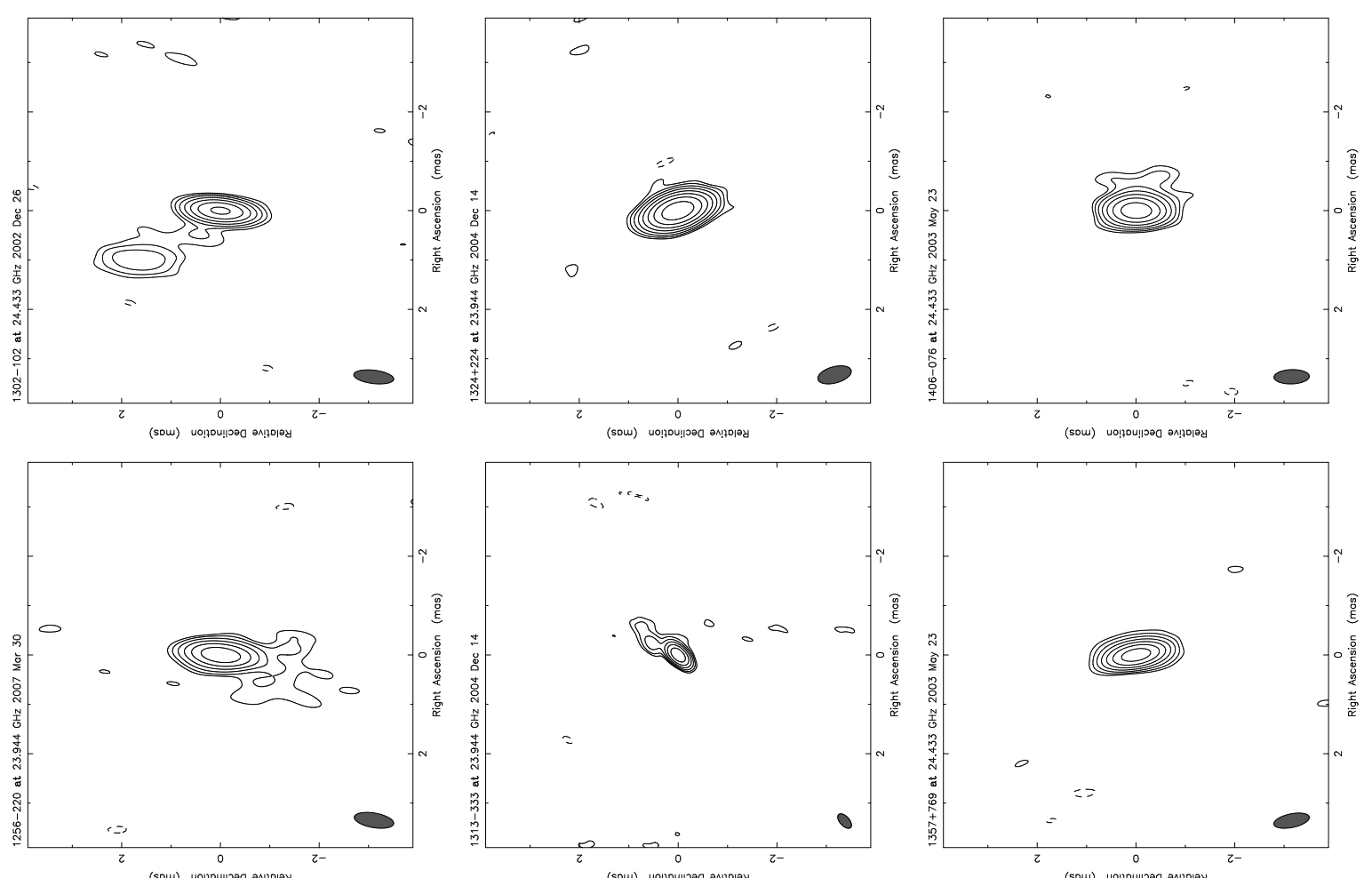

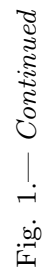
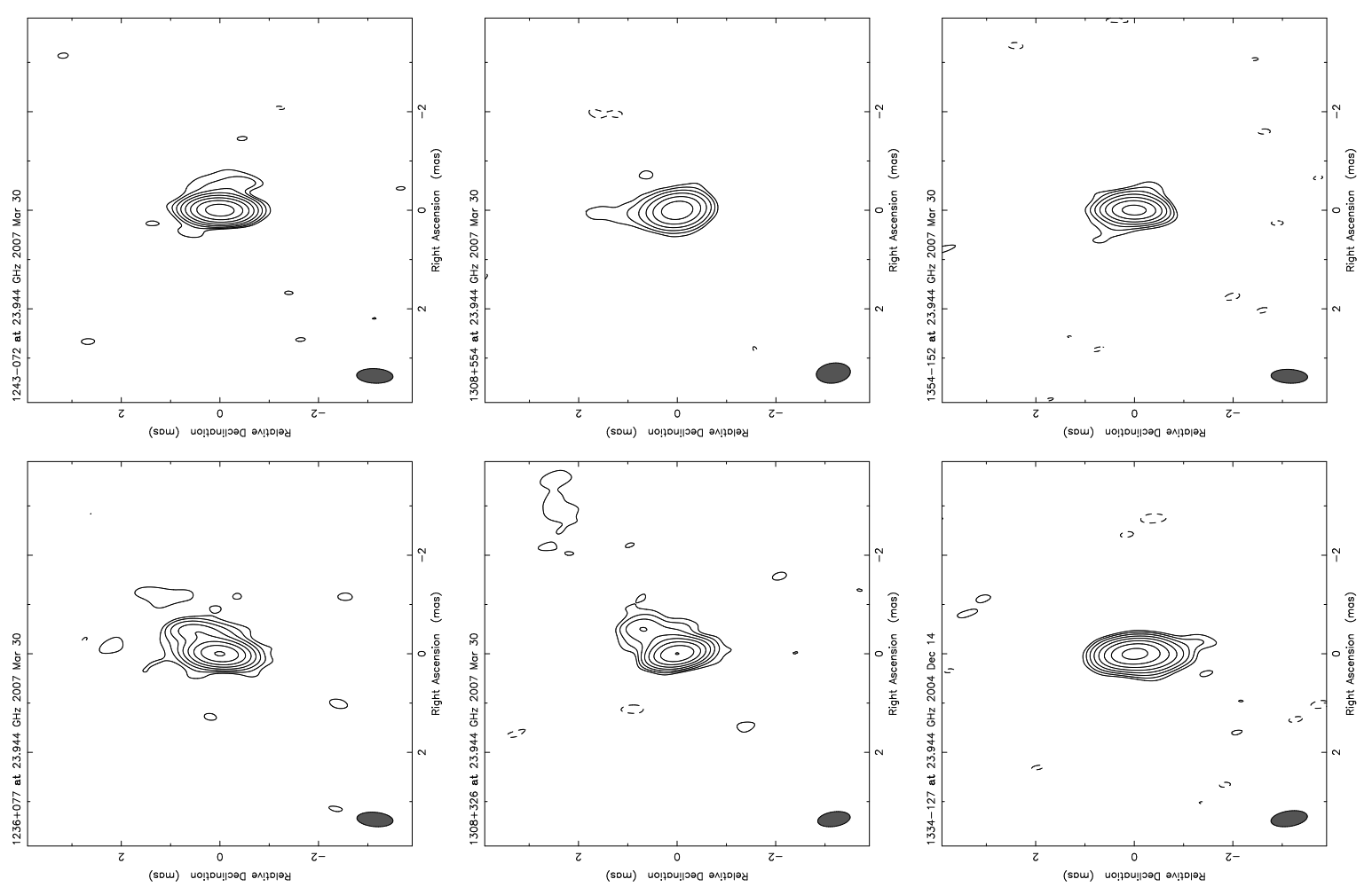
24

Charlot et al.
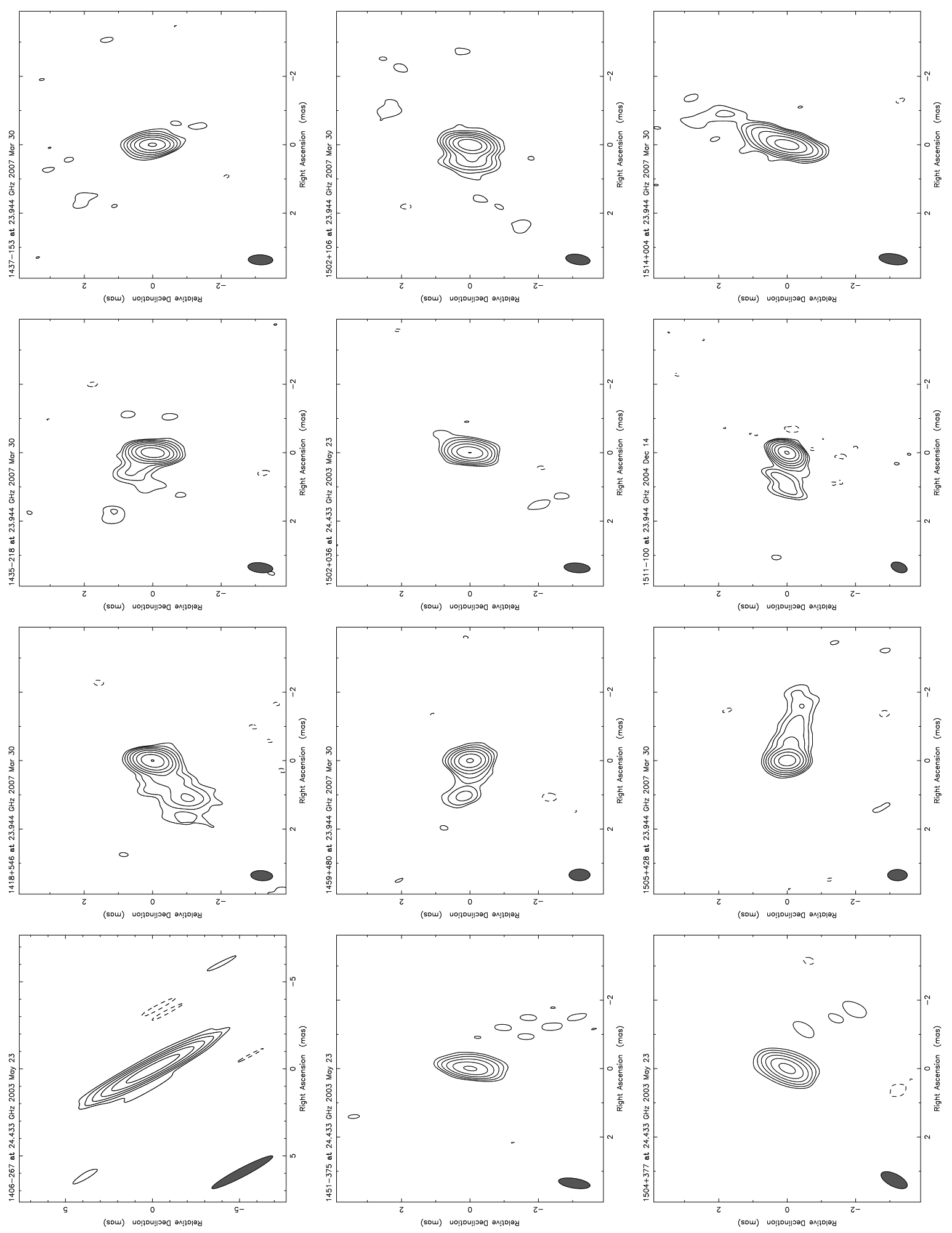

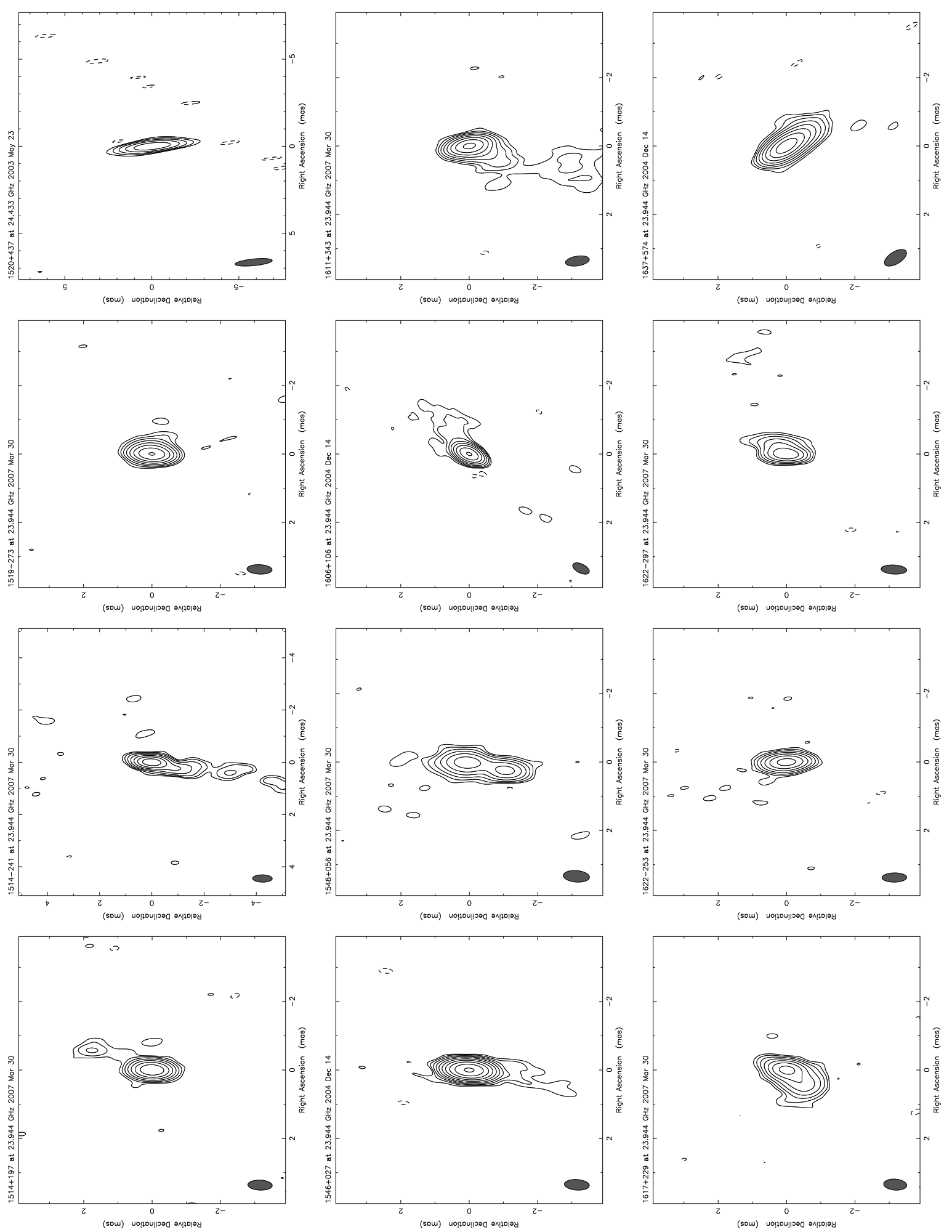

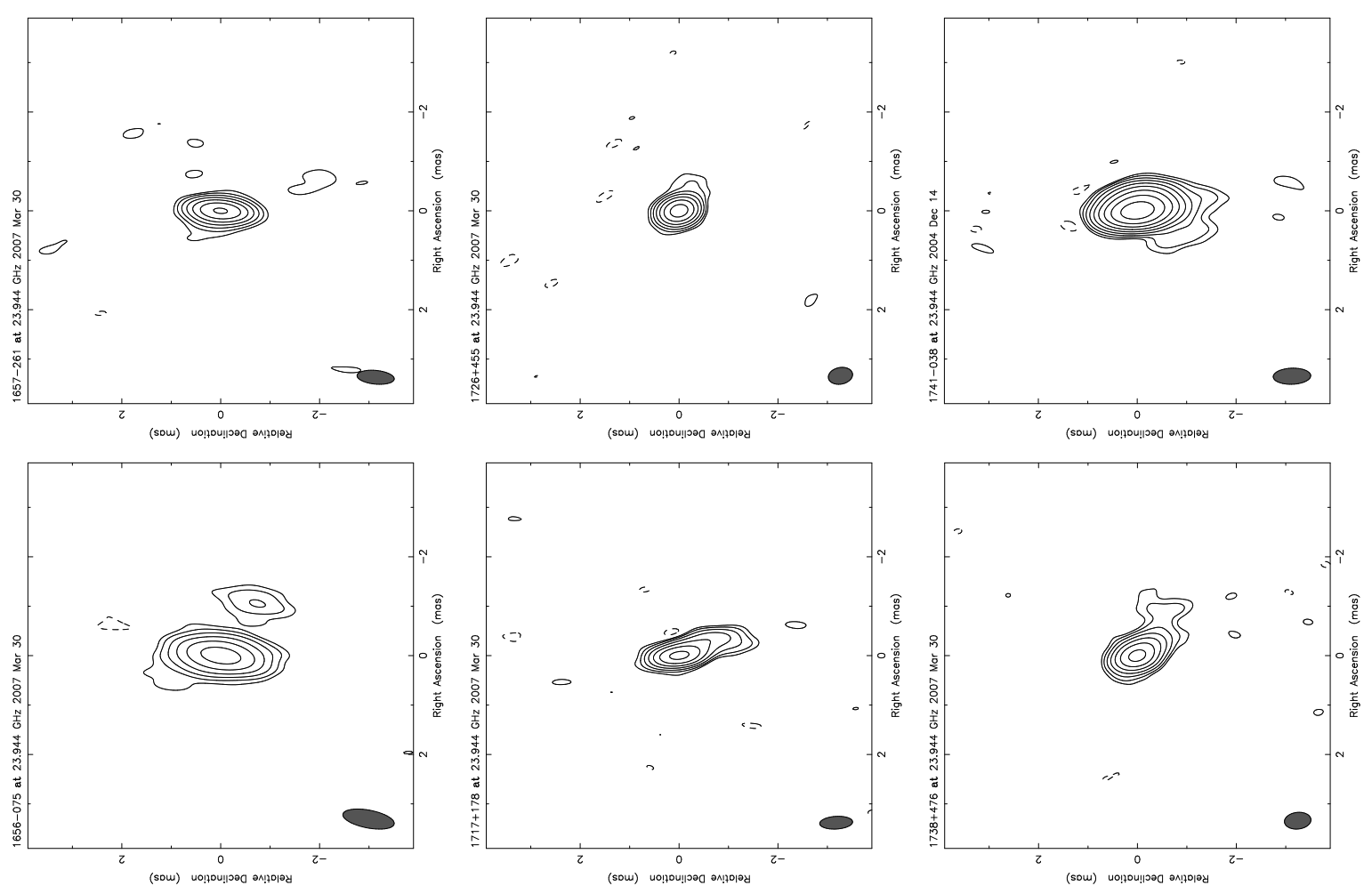

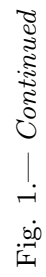
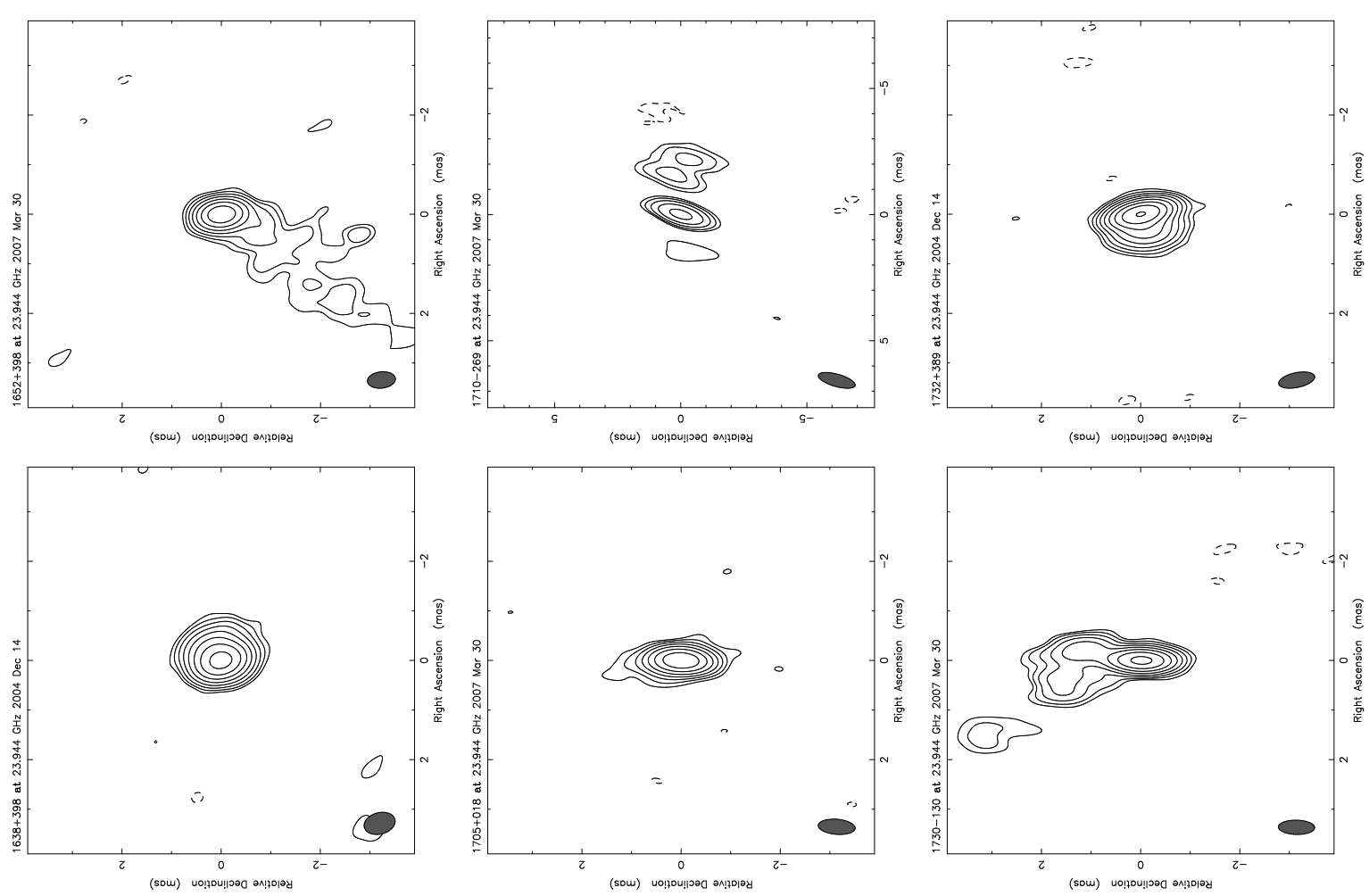

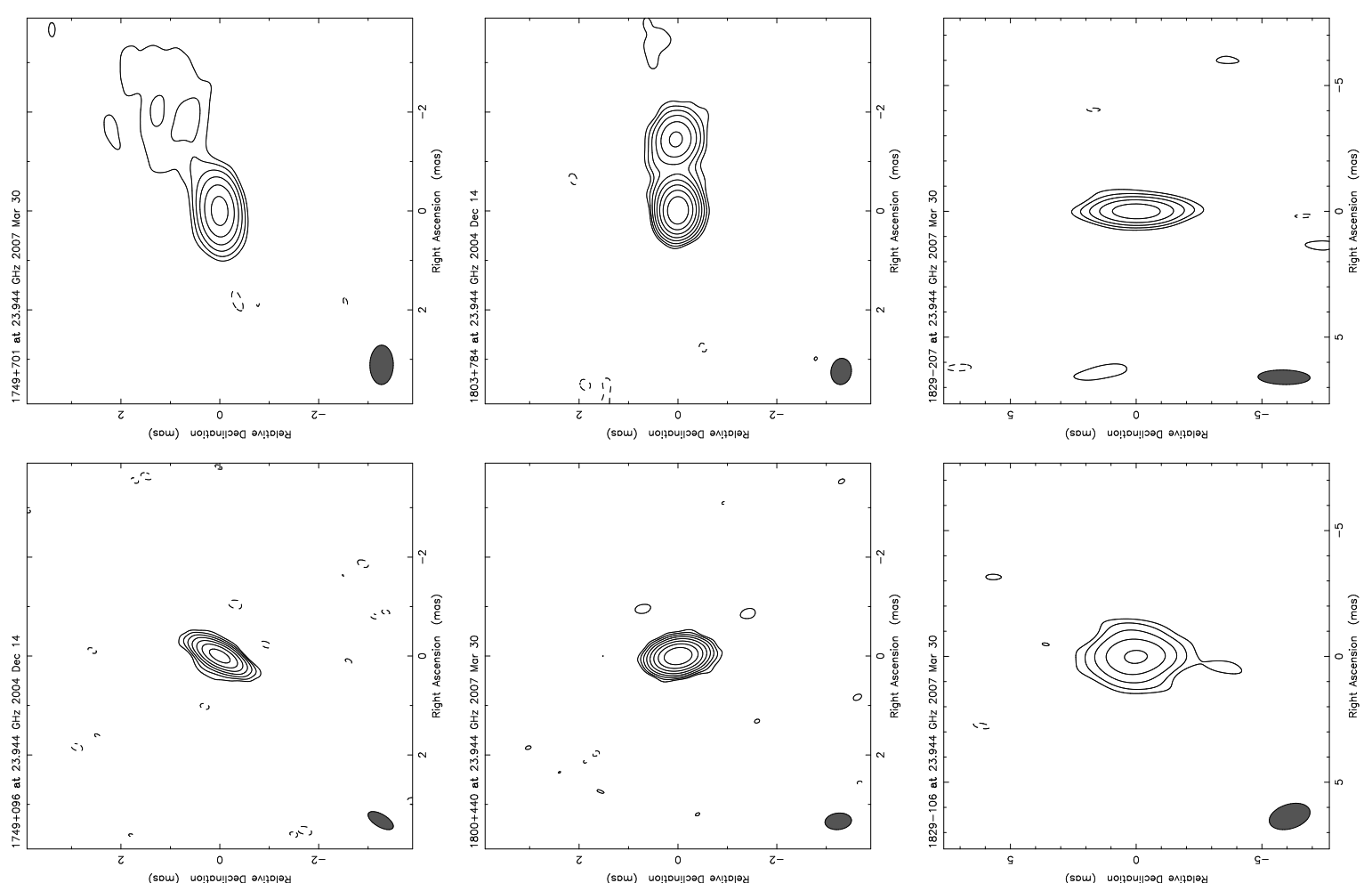

|.
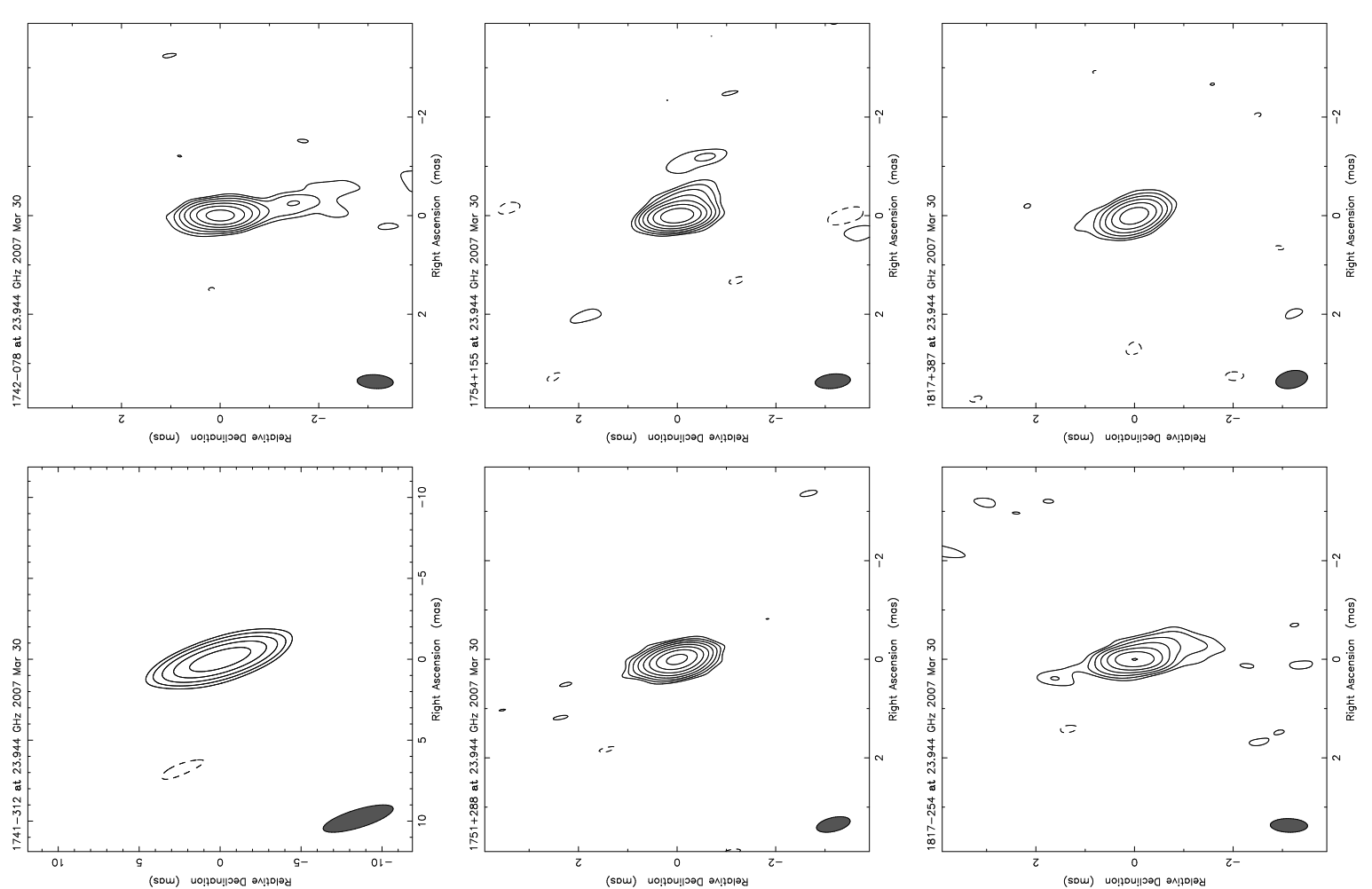
28

Charlot et al.
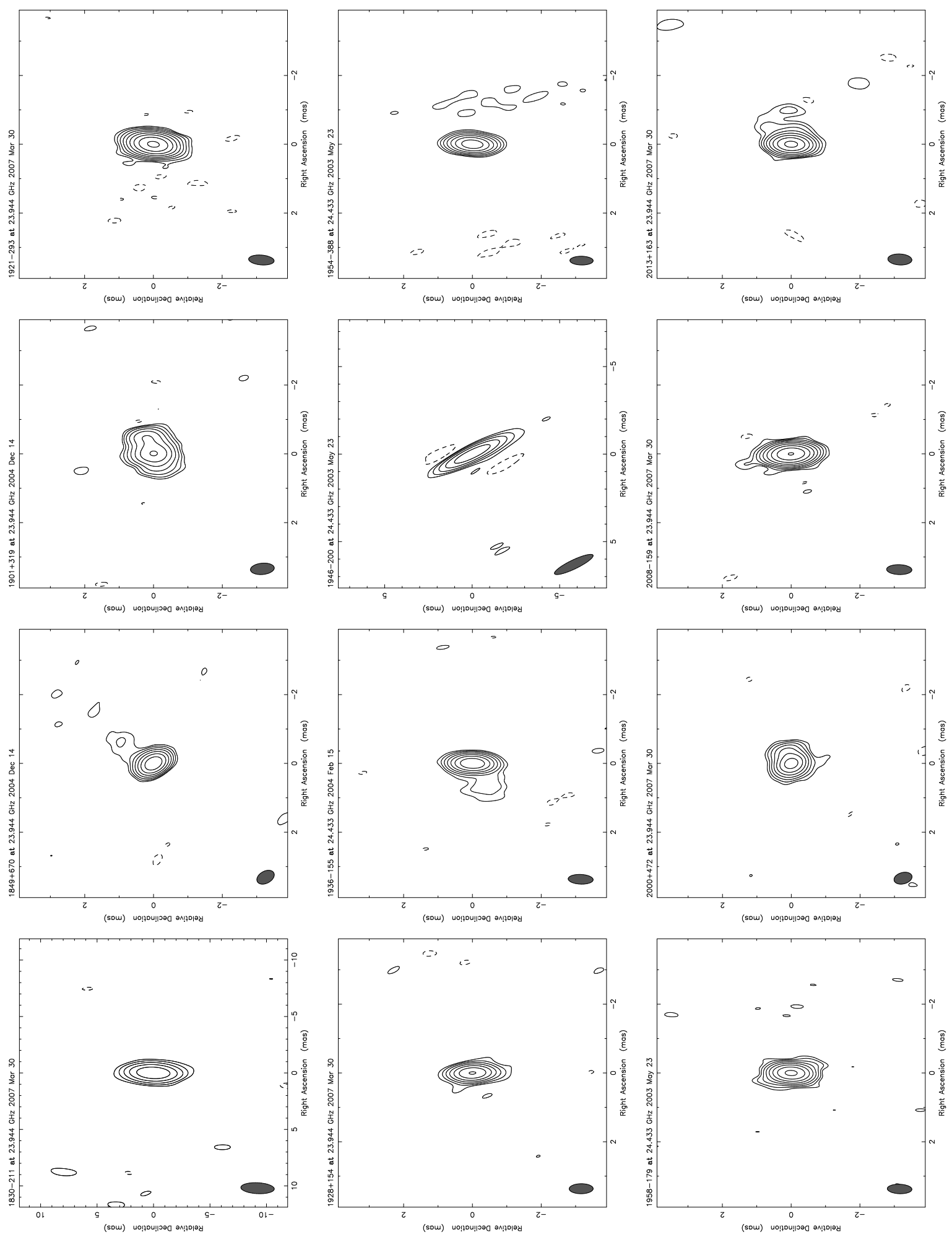

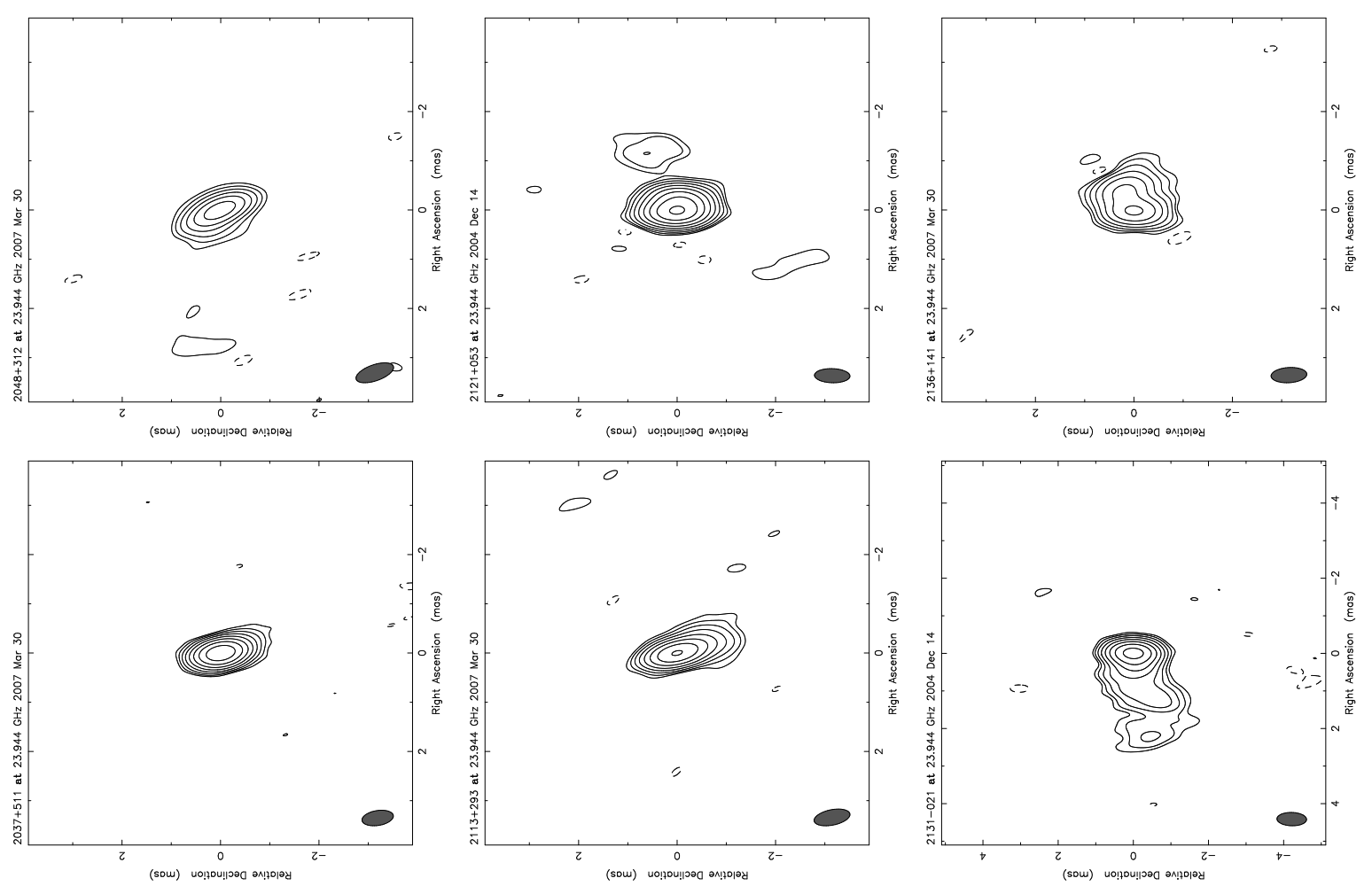

ن.
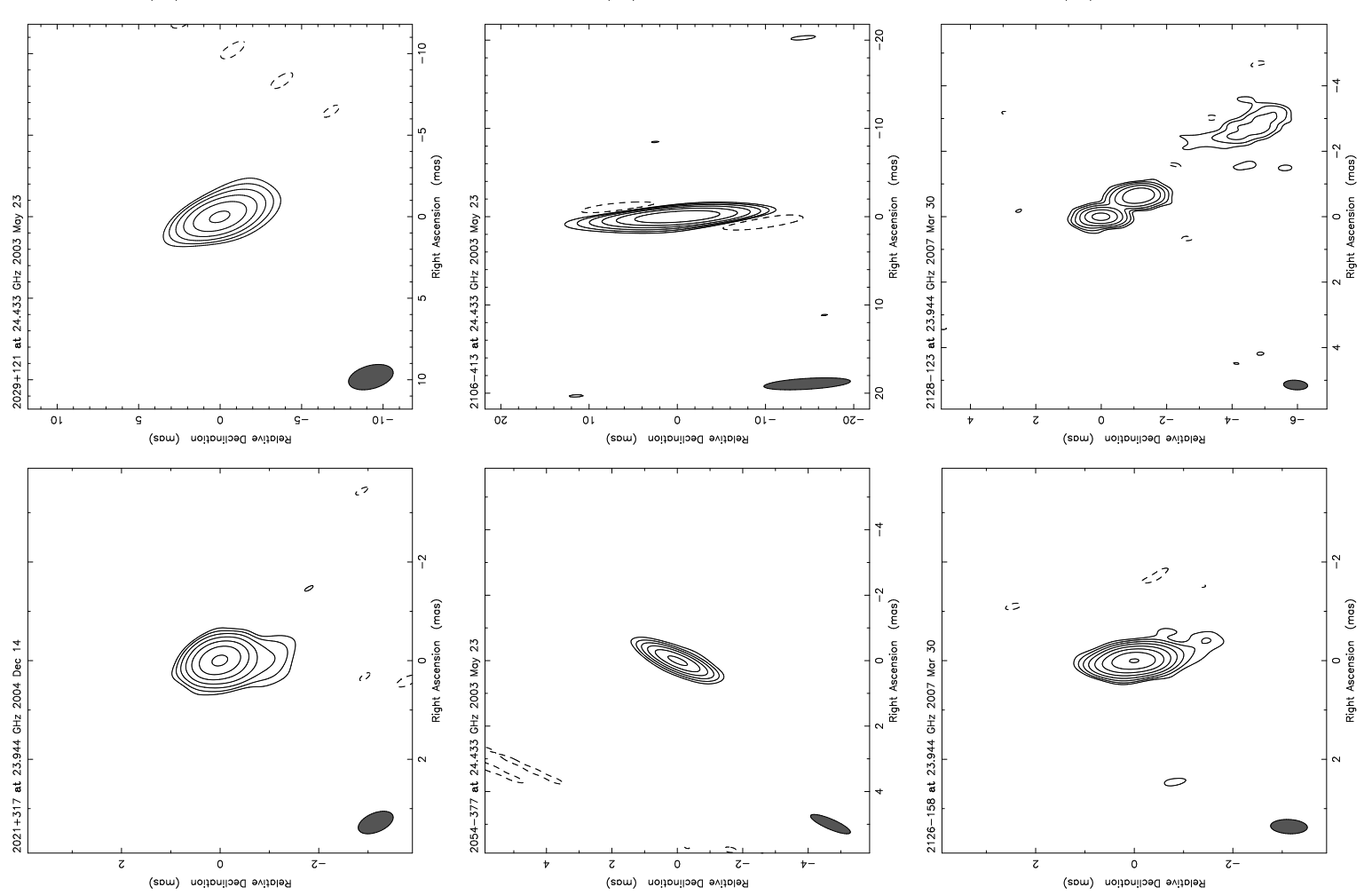
30

Charlot et al.
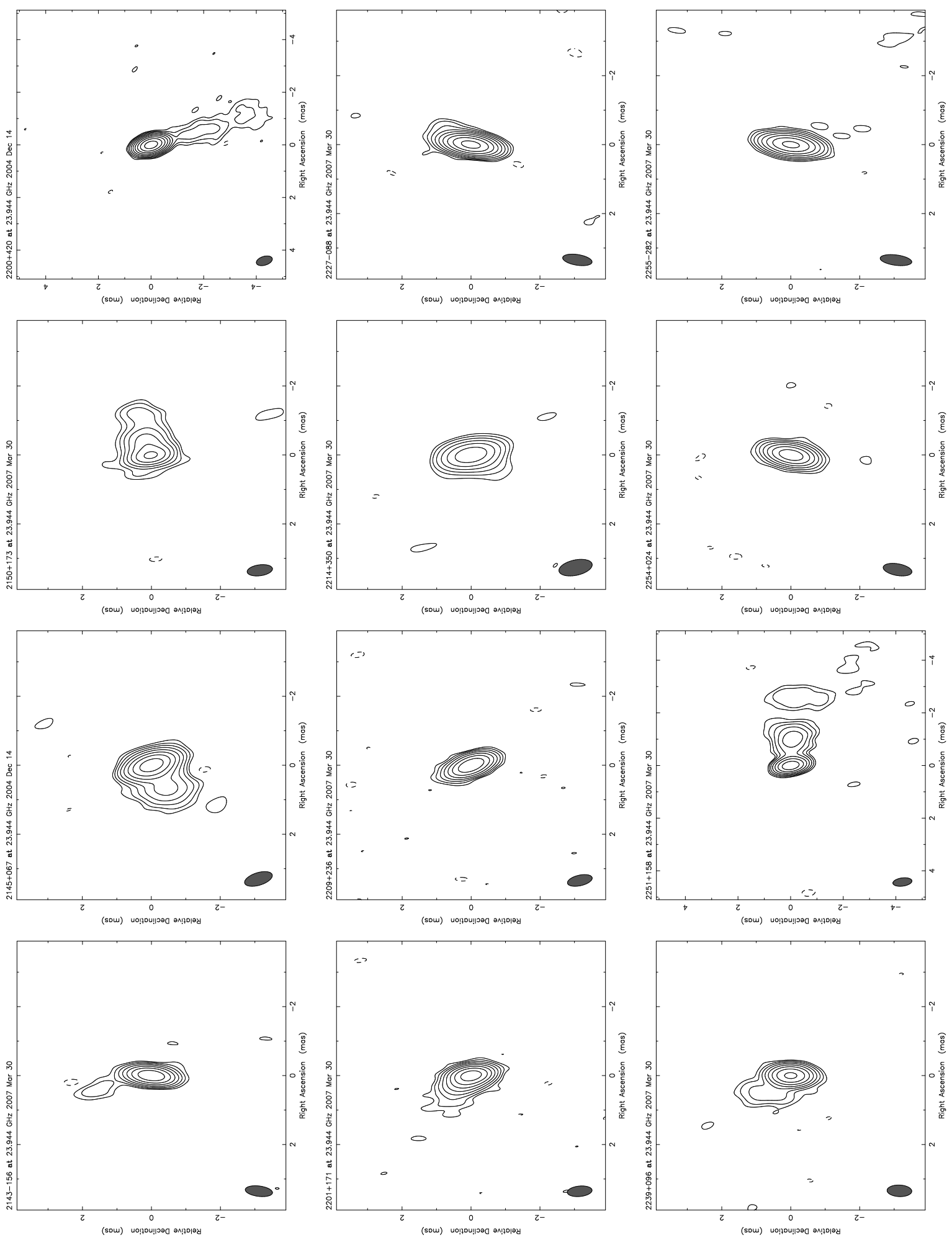

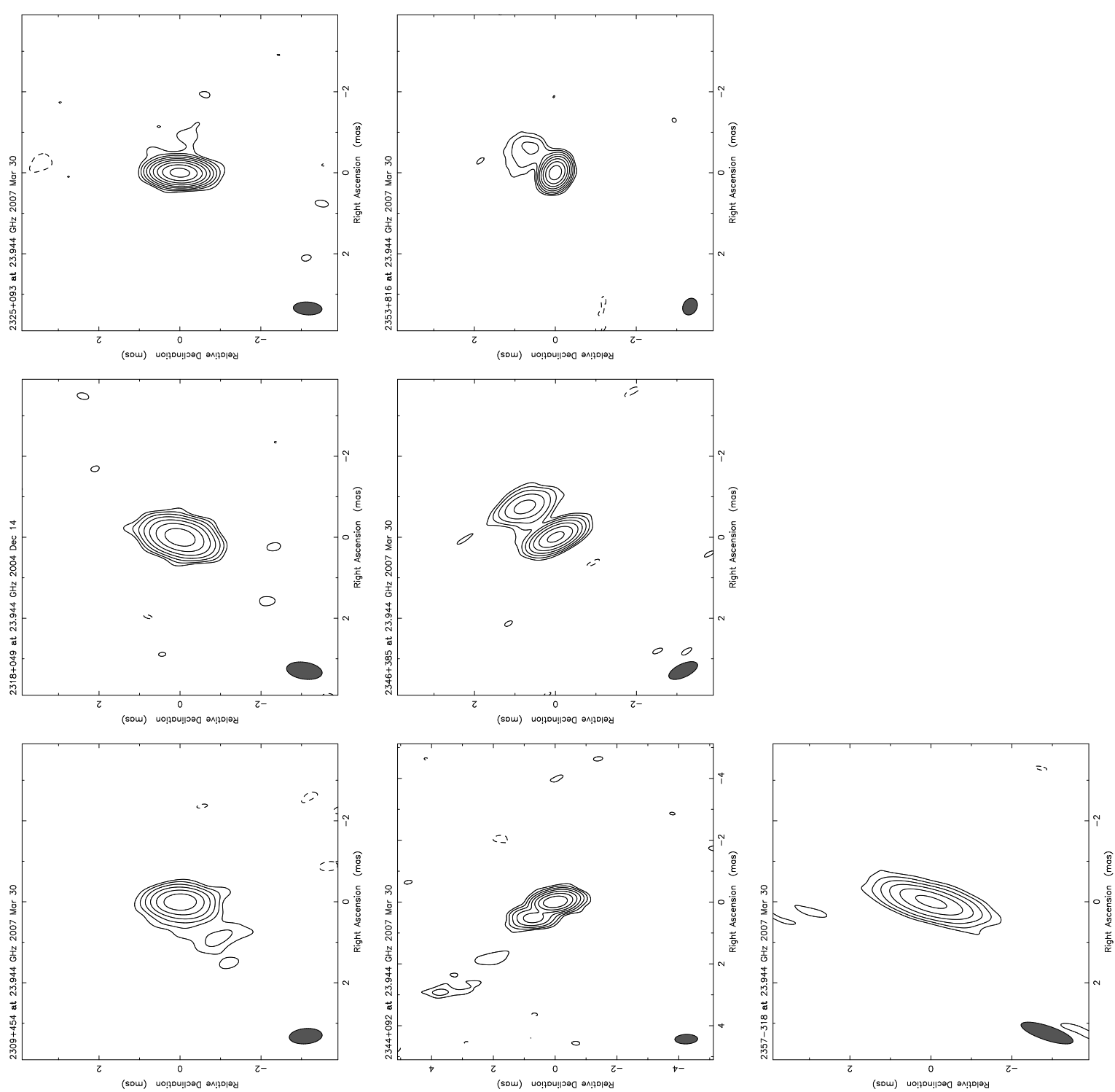

ن்
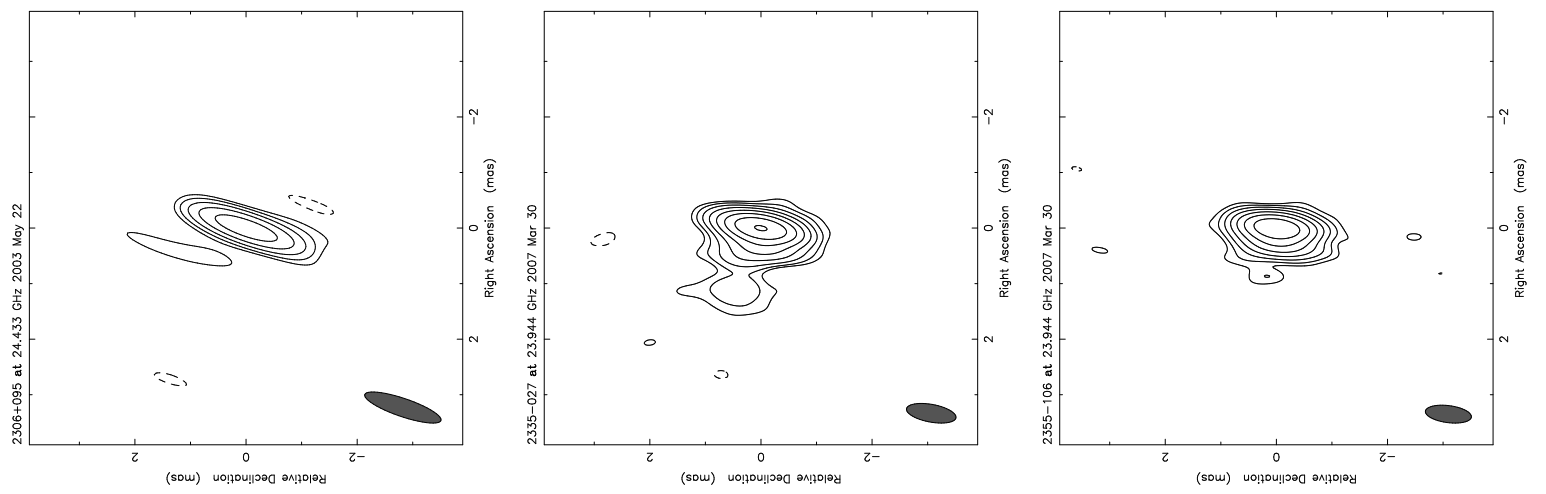
32

Charlot et al.
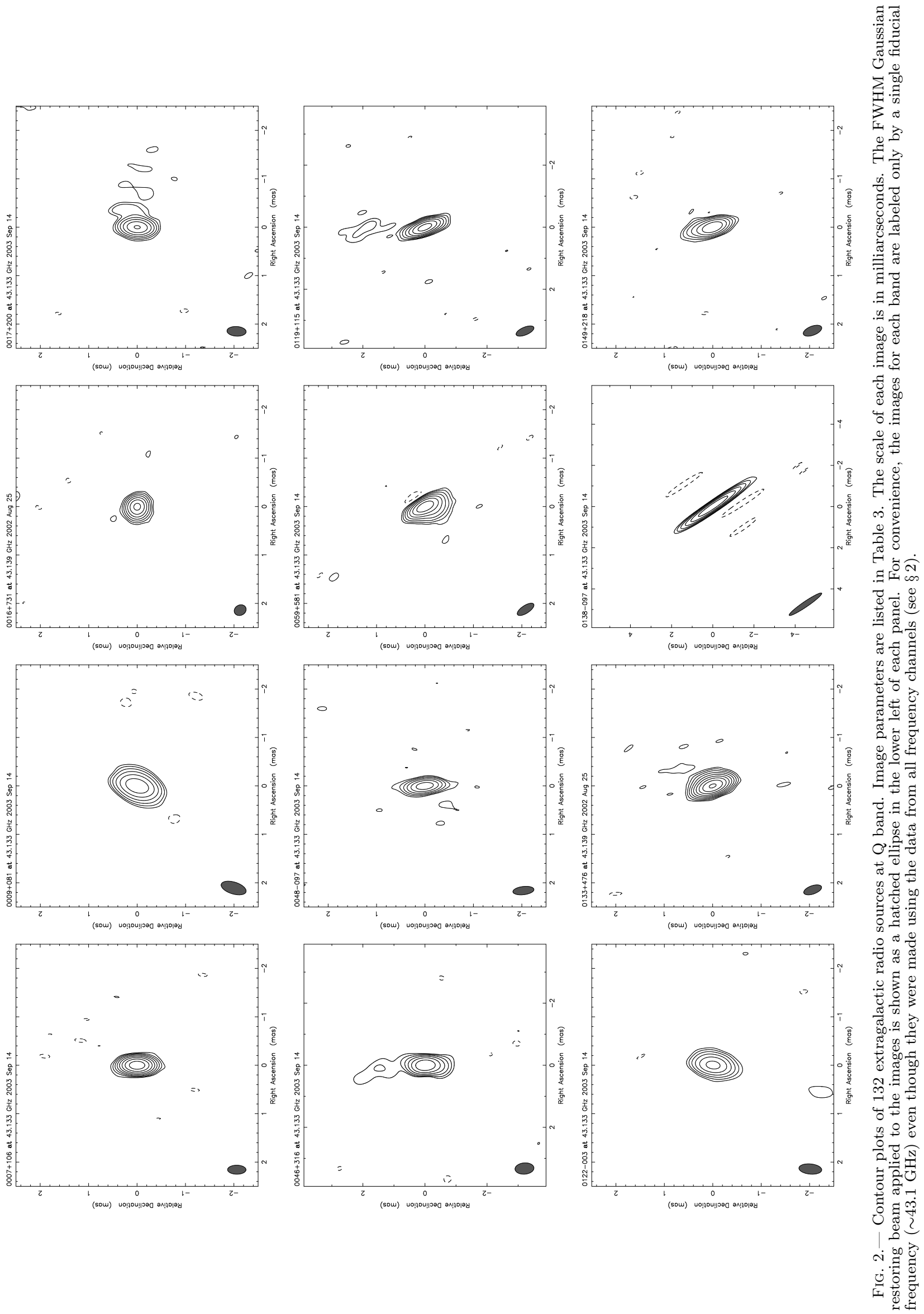

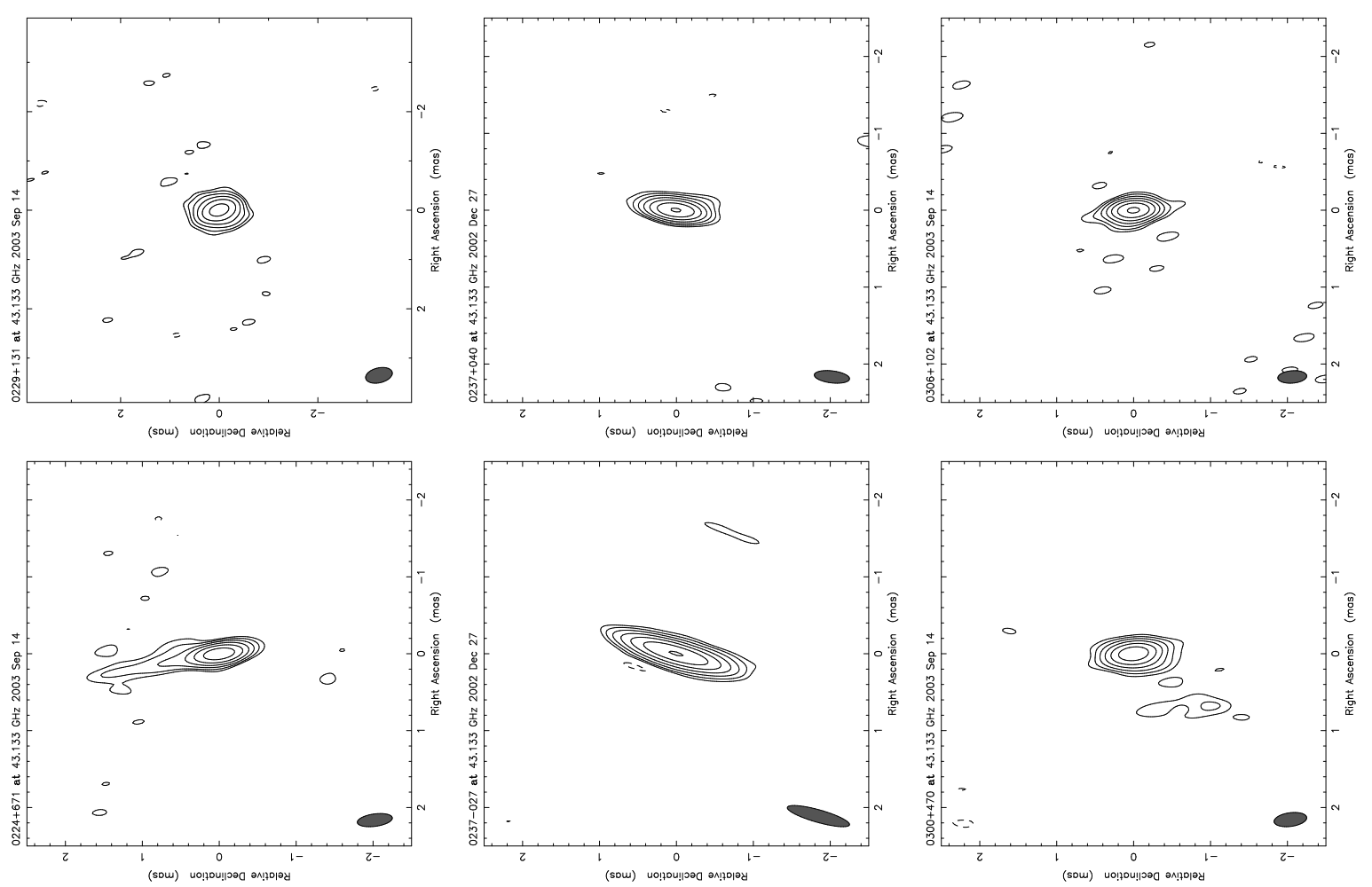

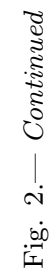
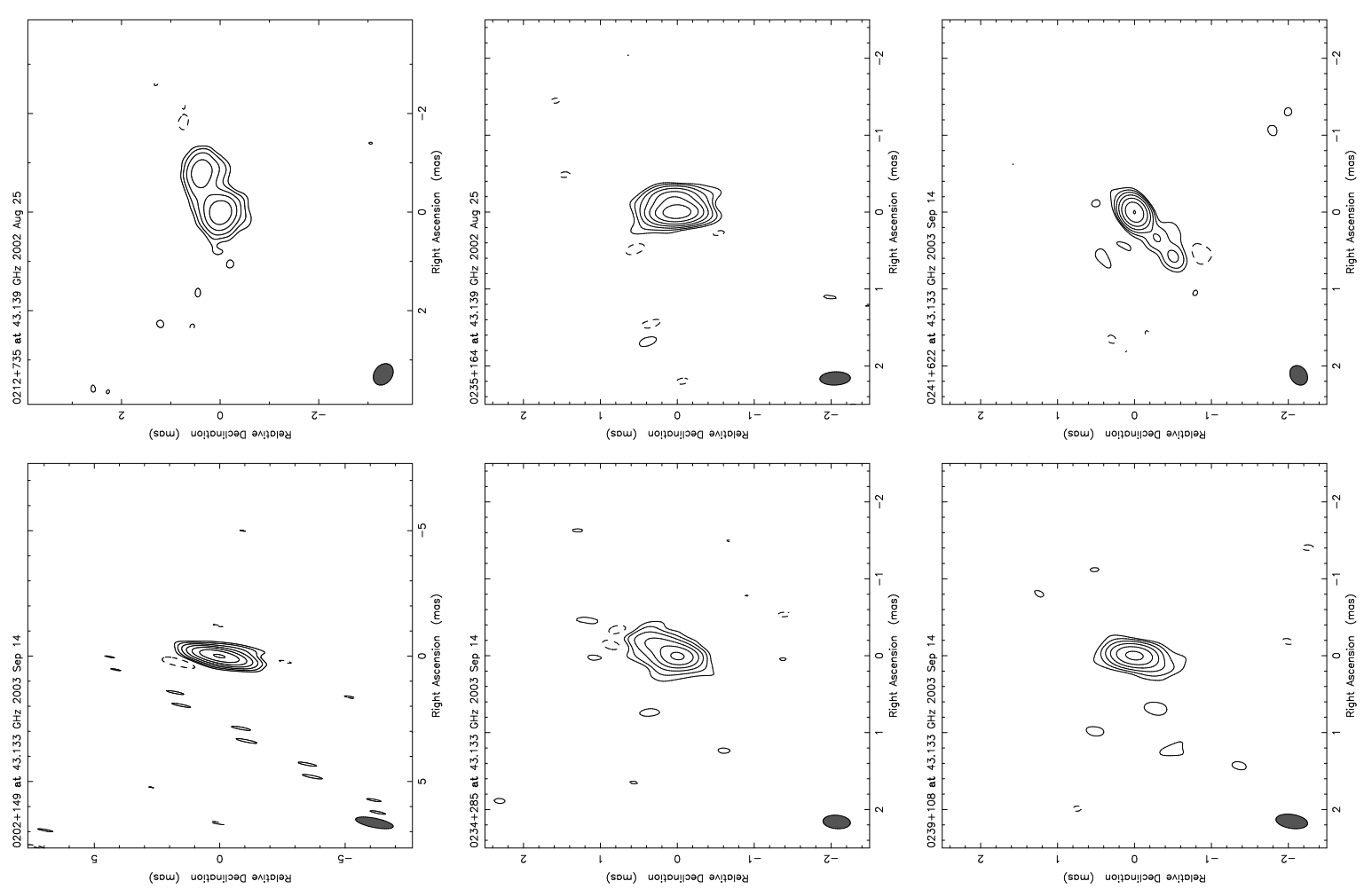

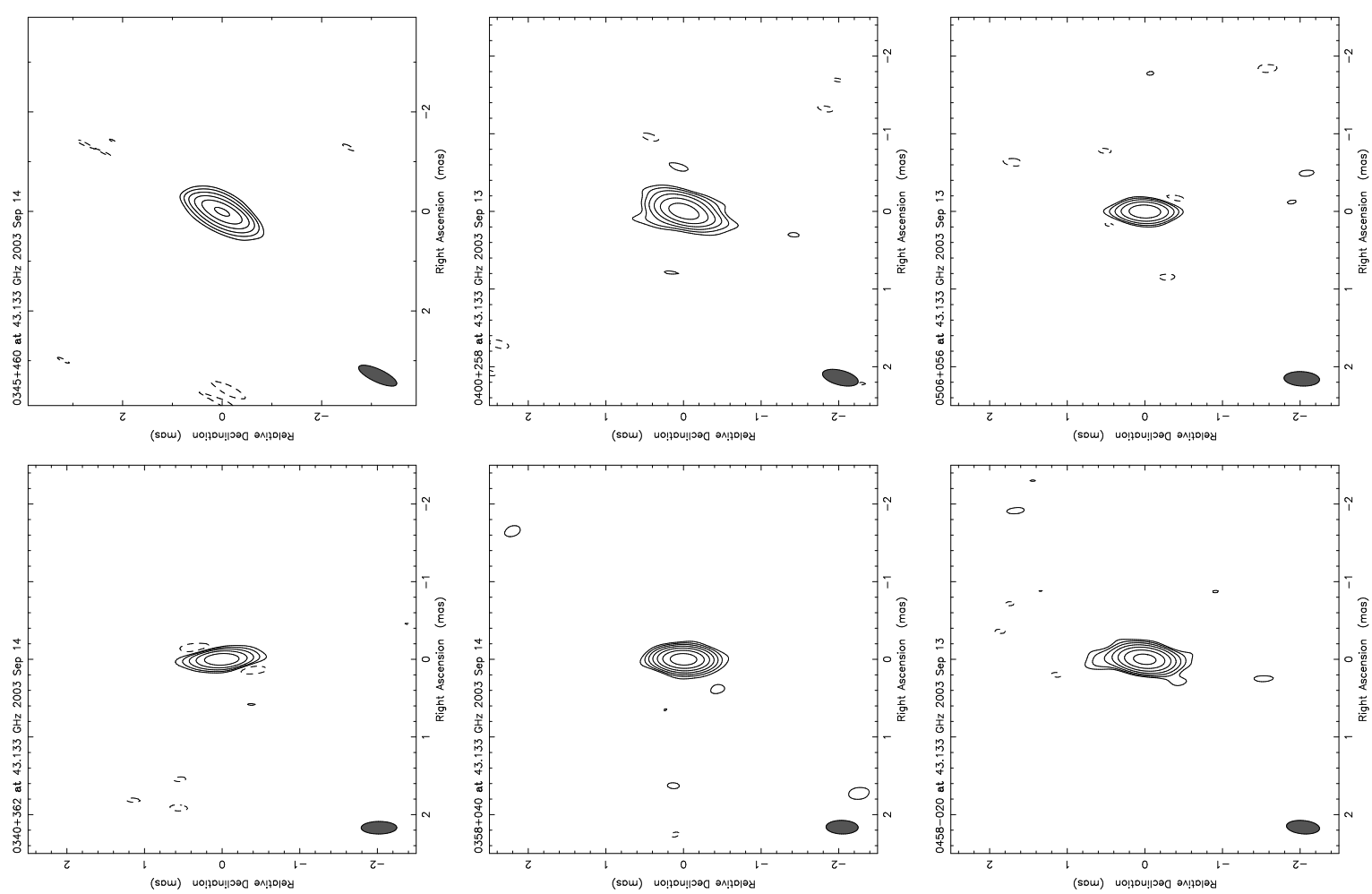

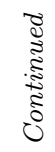
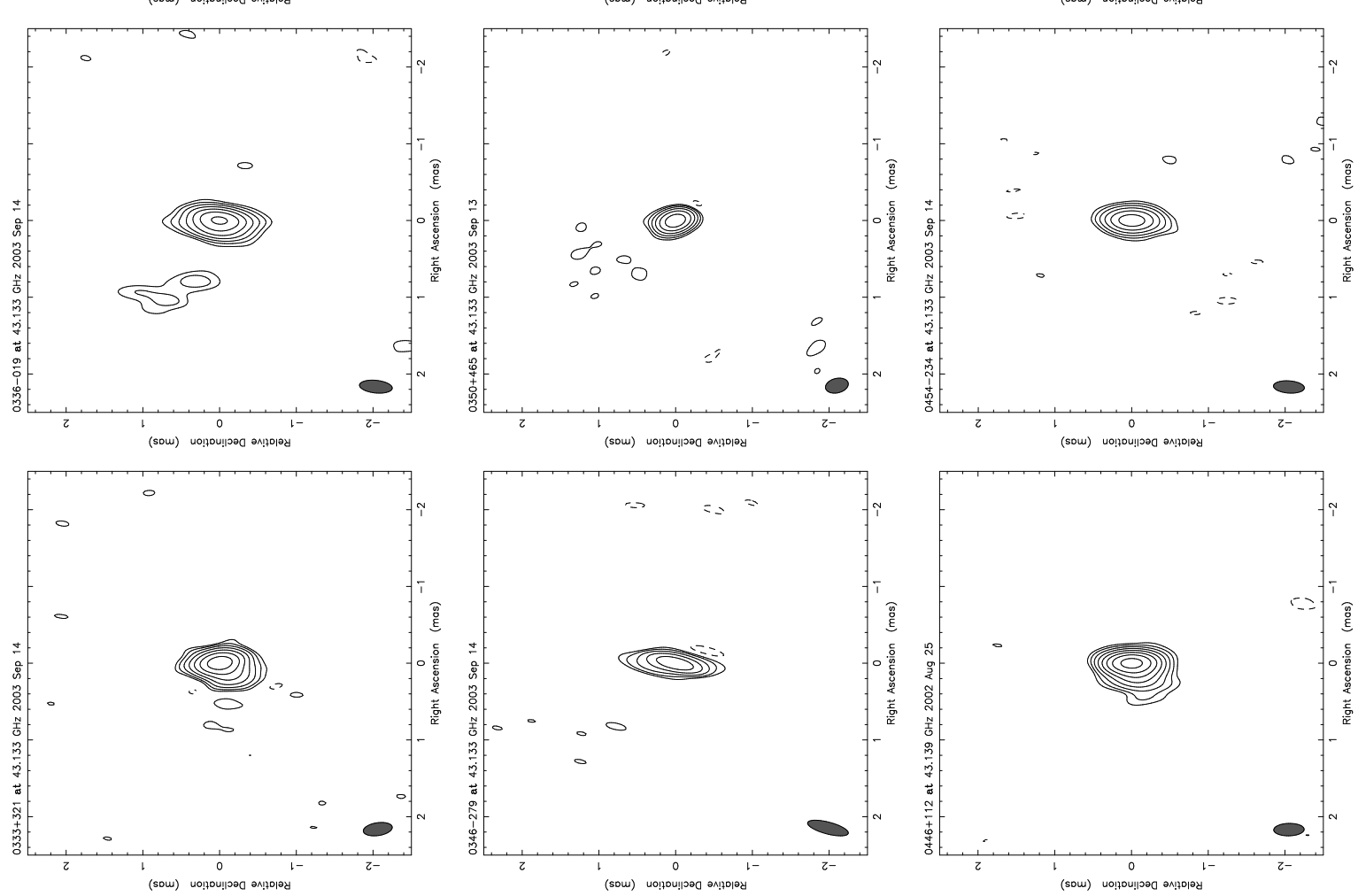

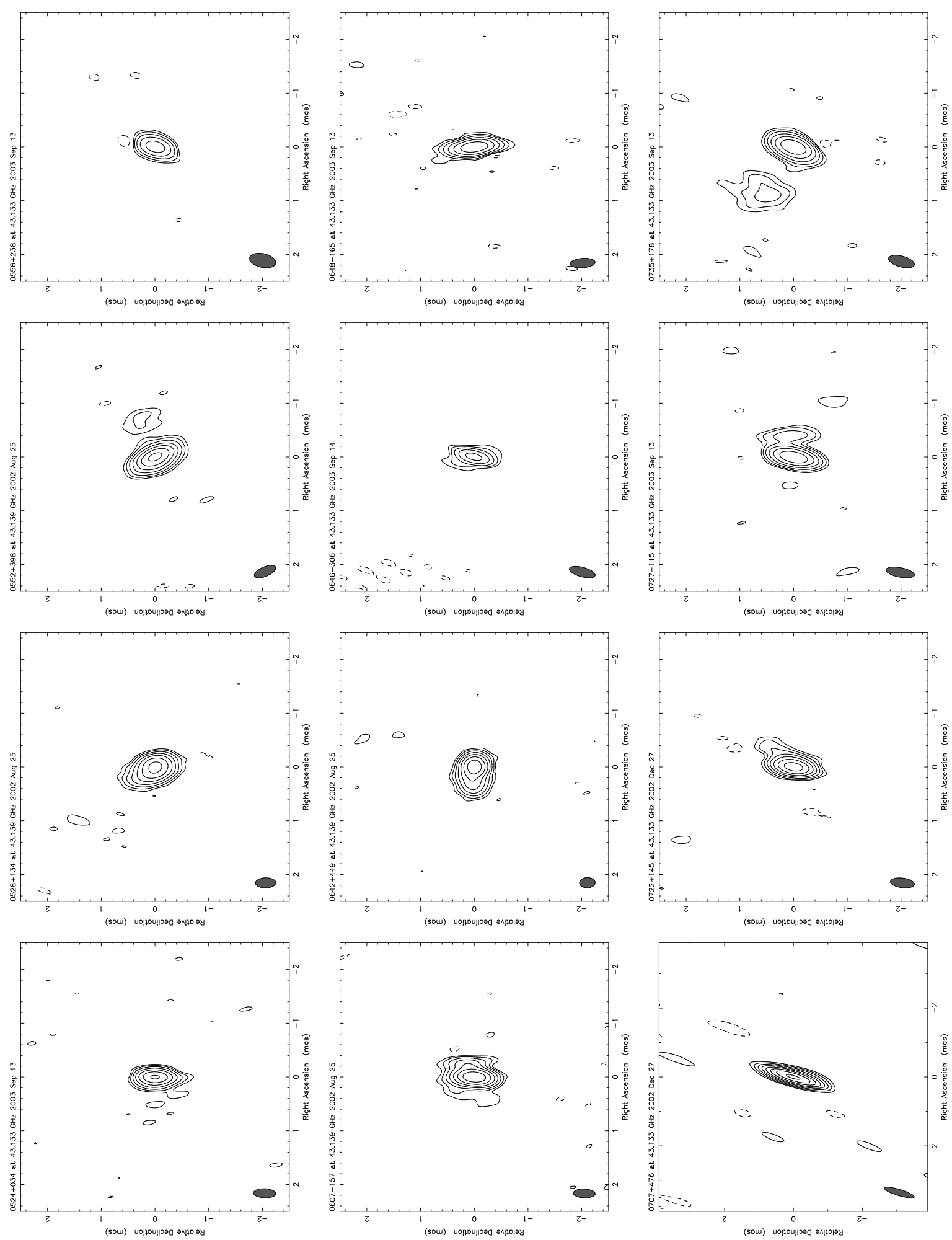

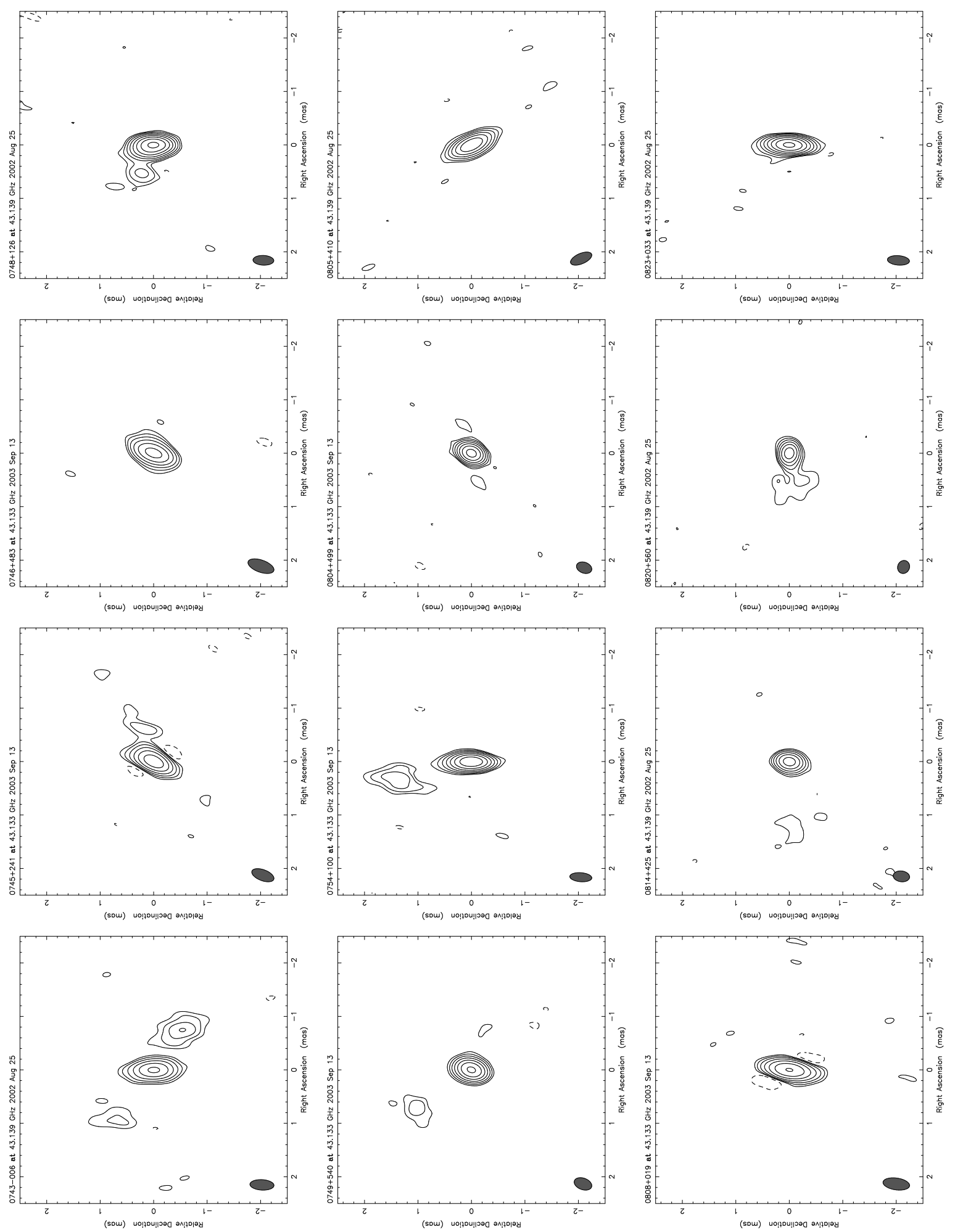

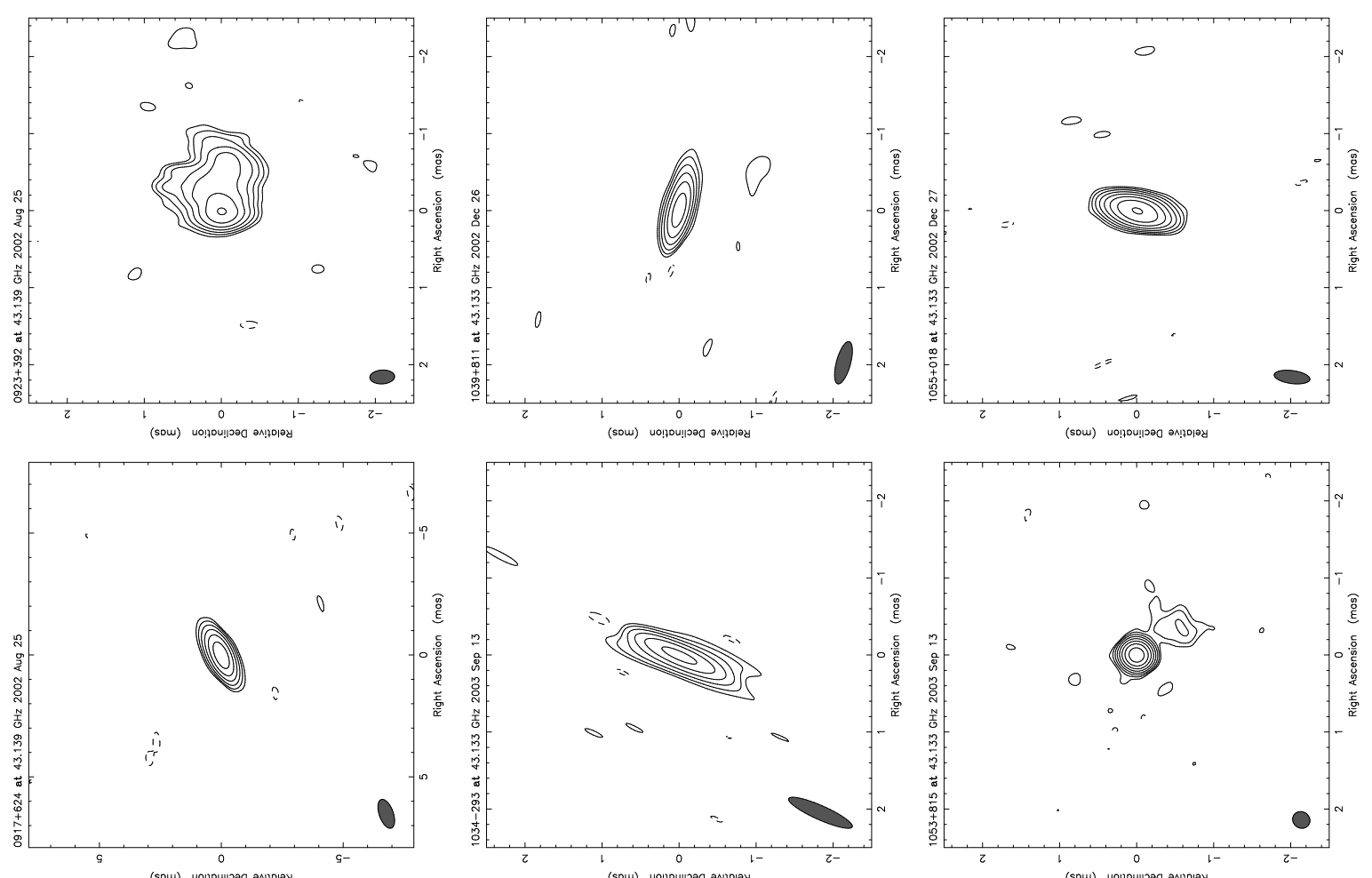

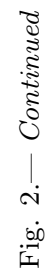
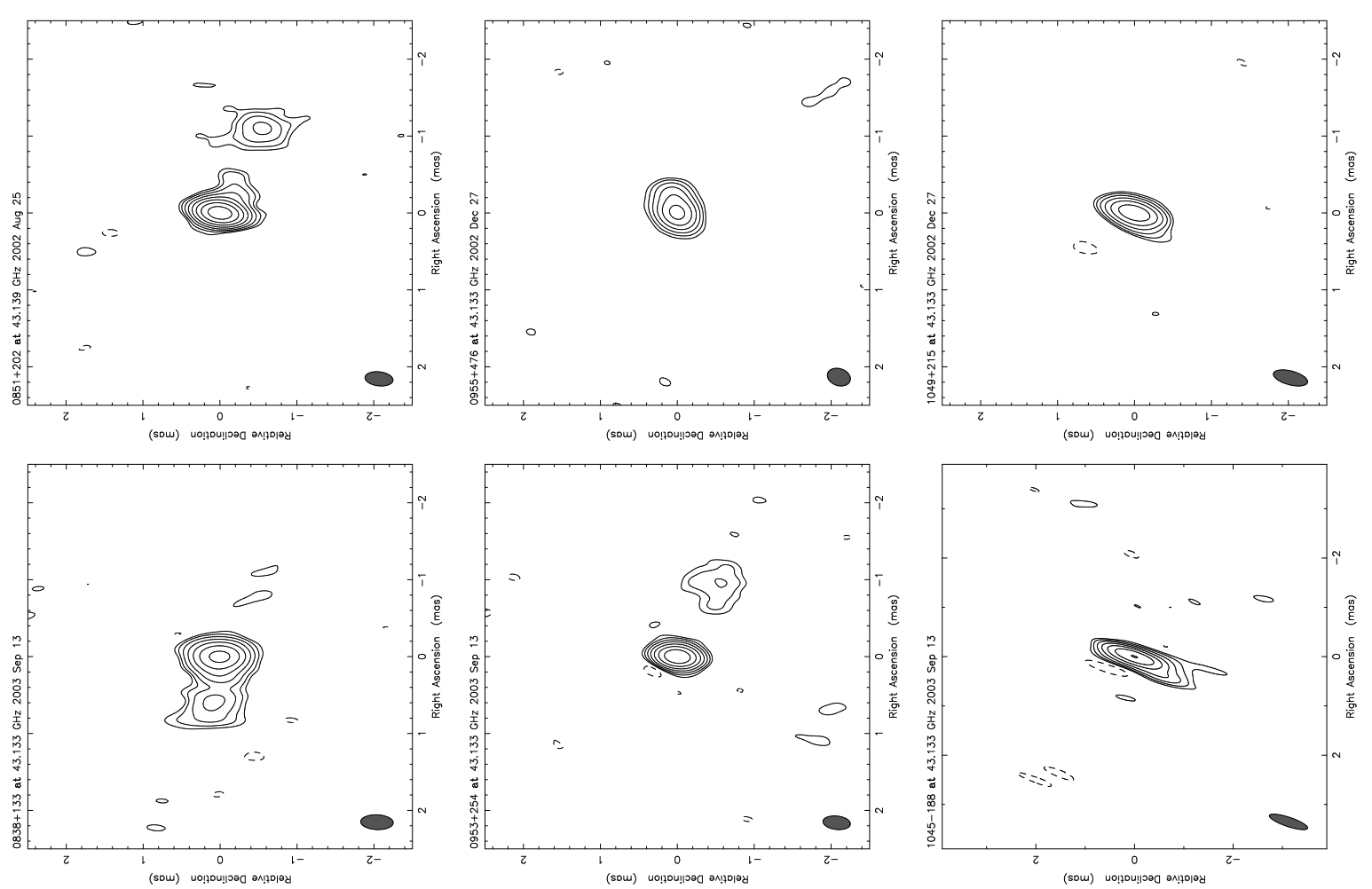

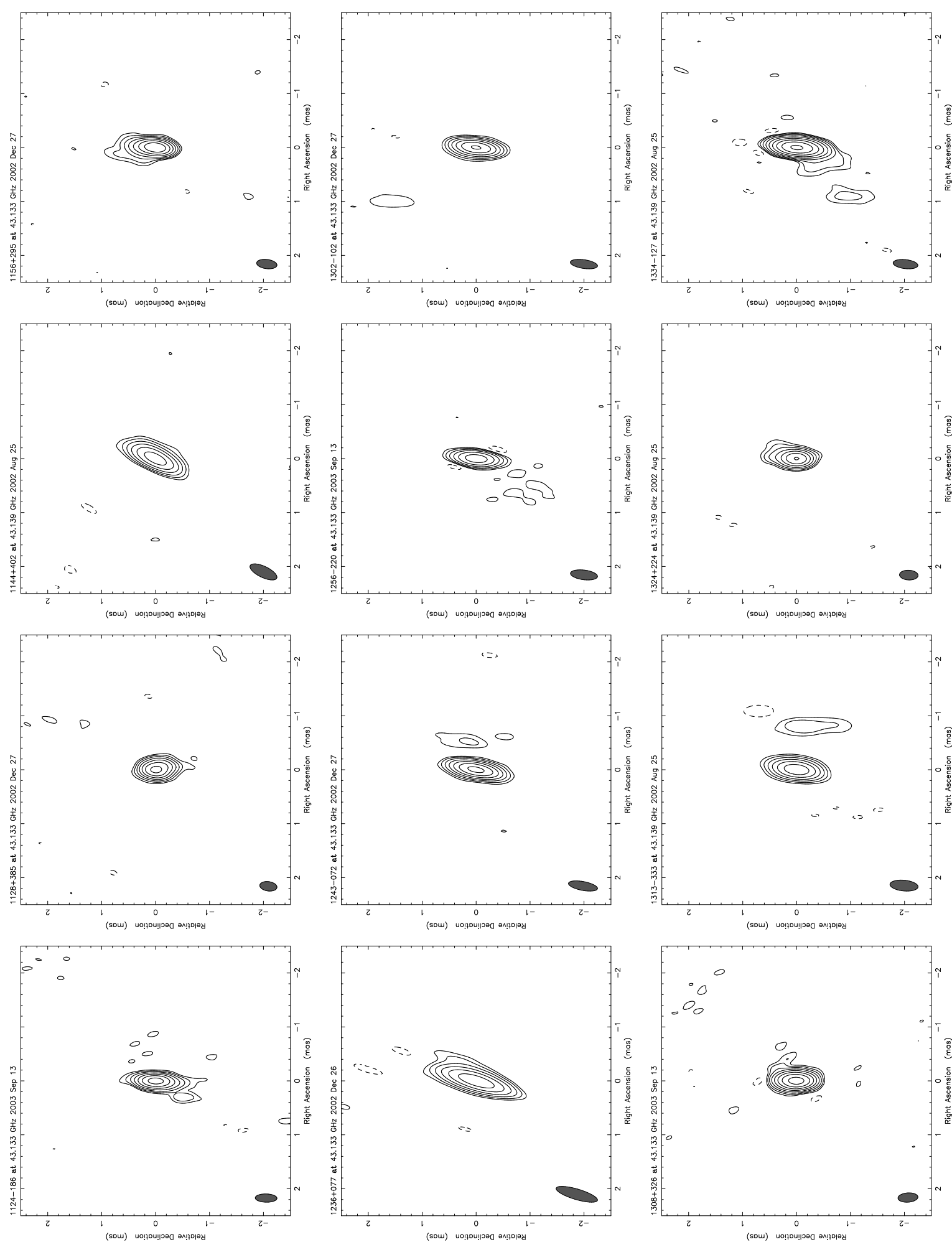

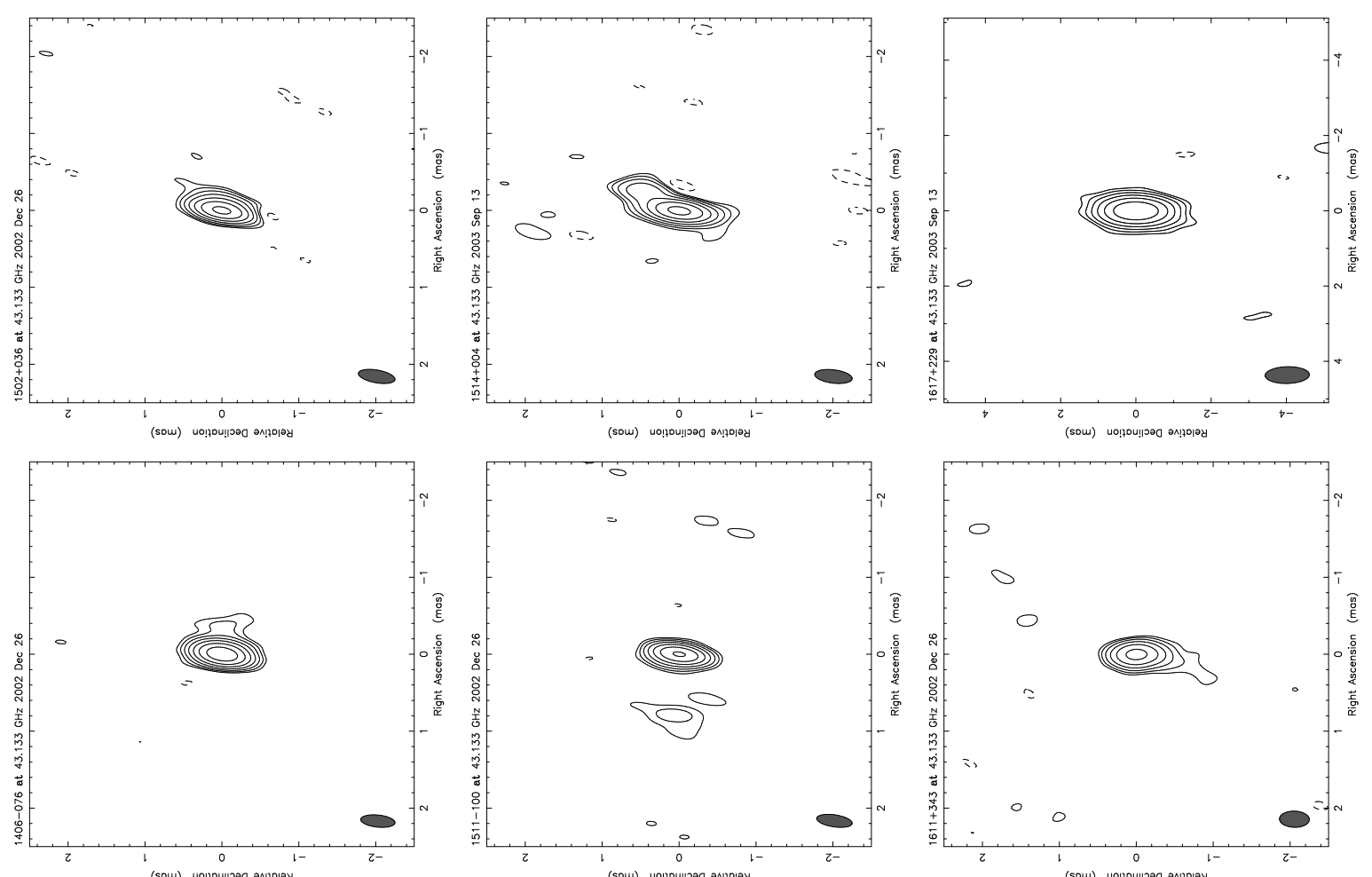

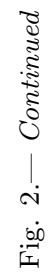
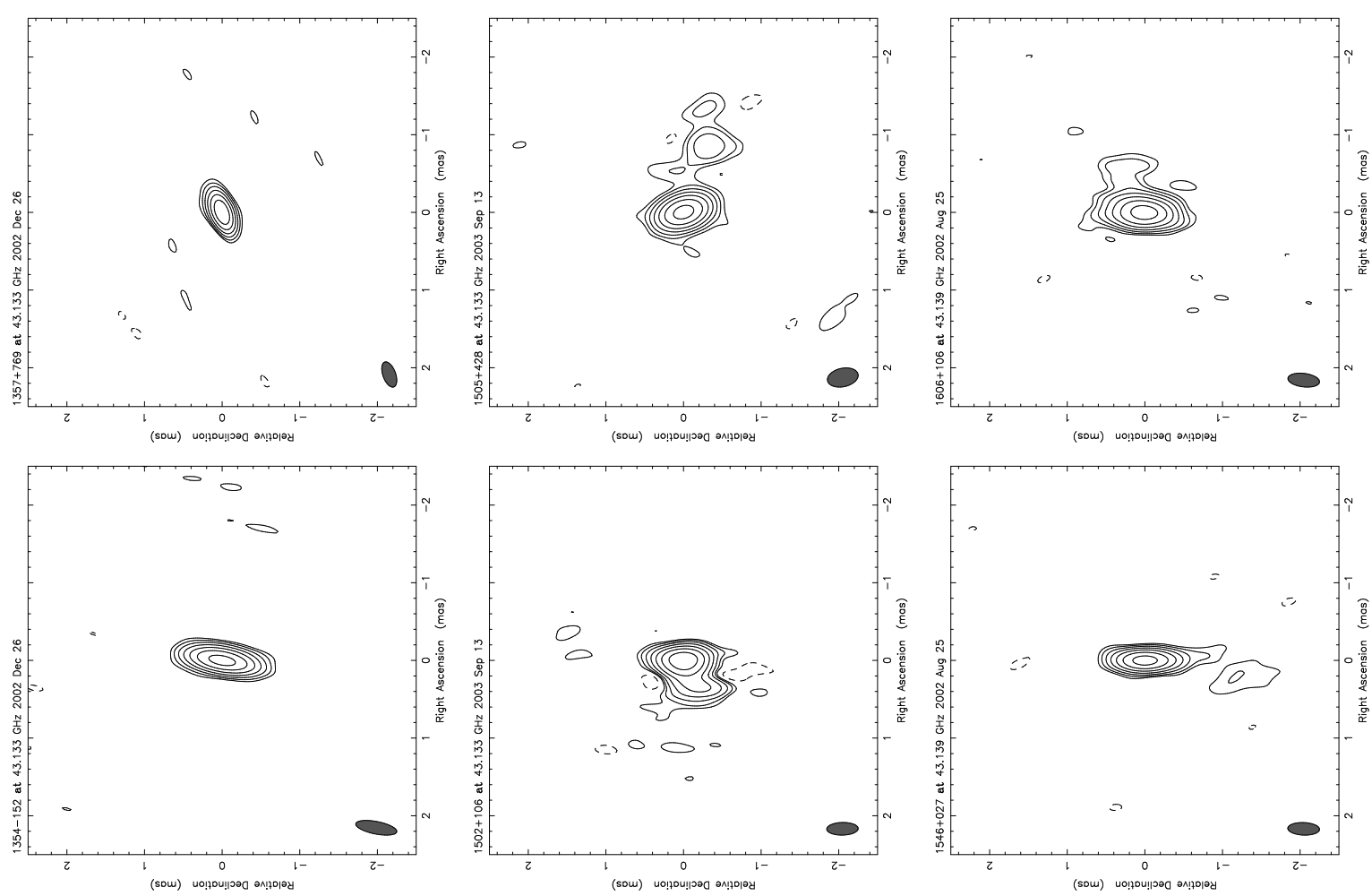

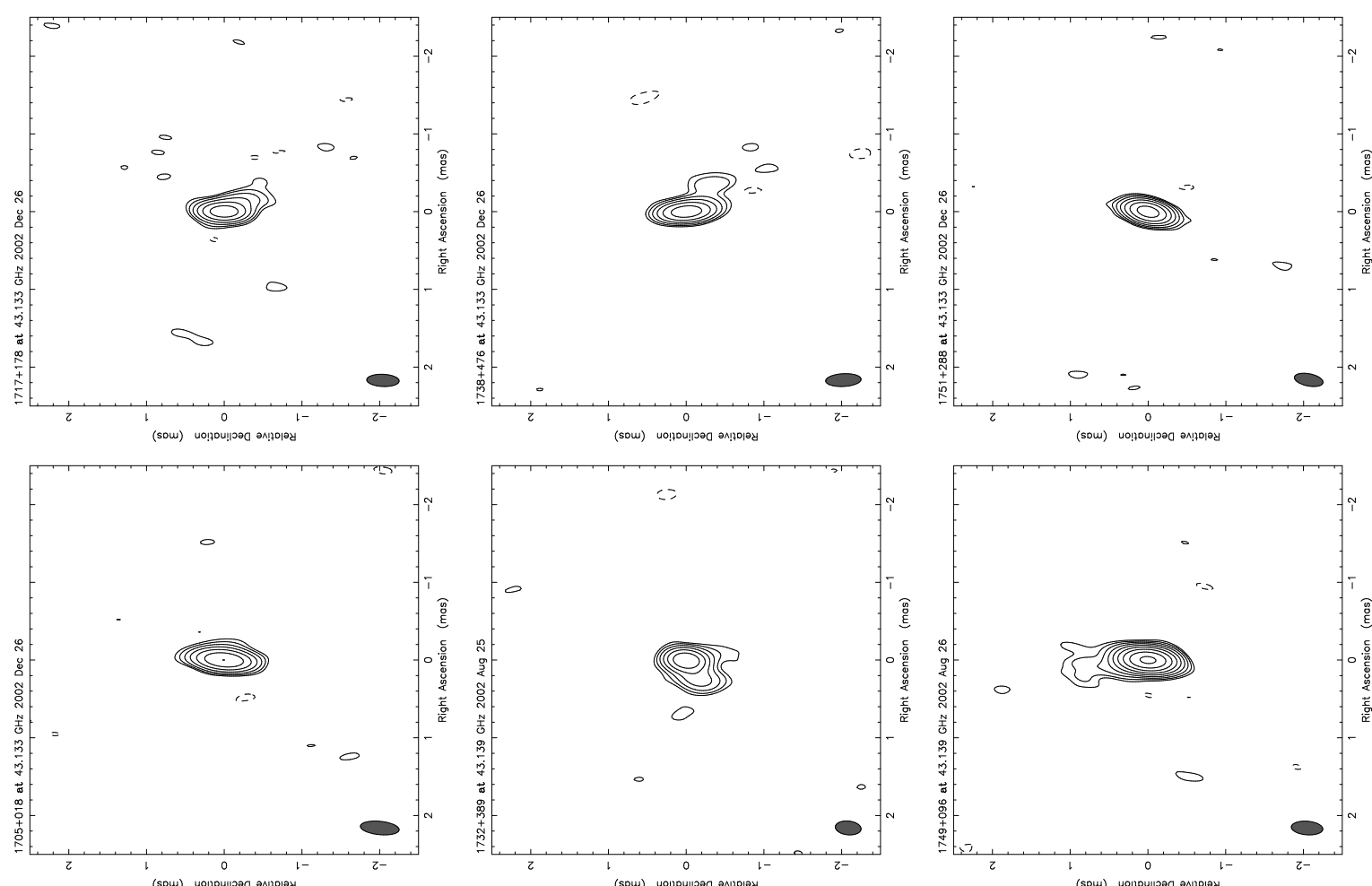

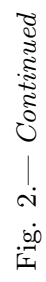
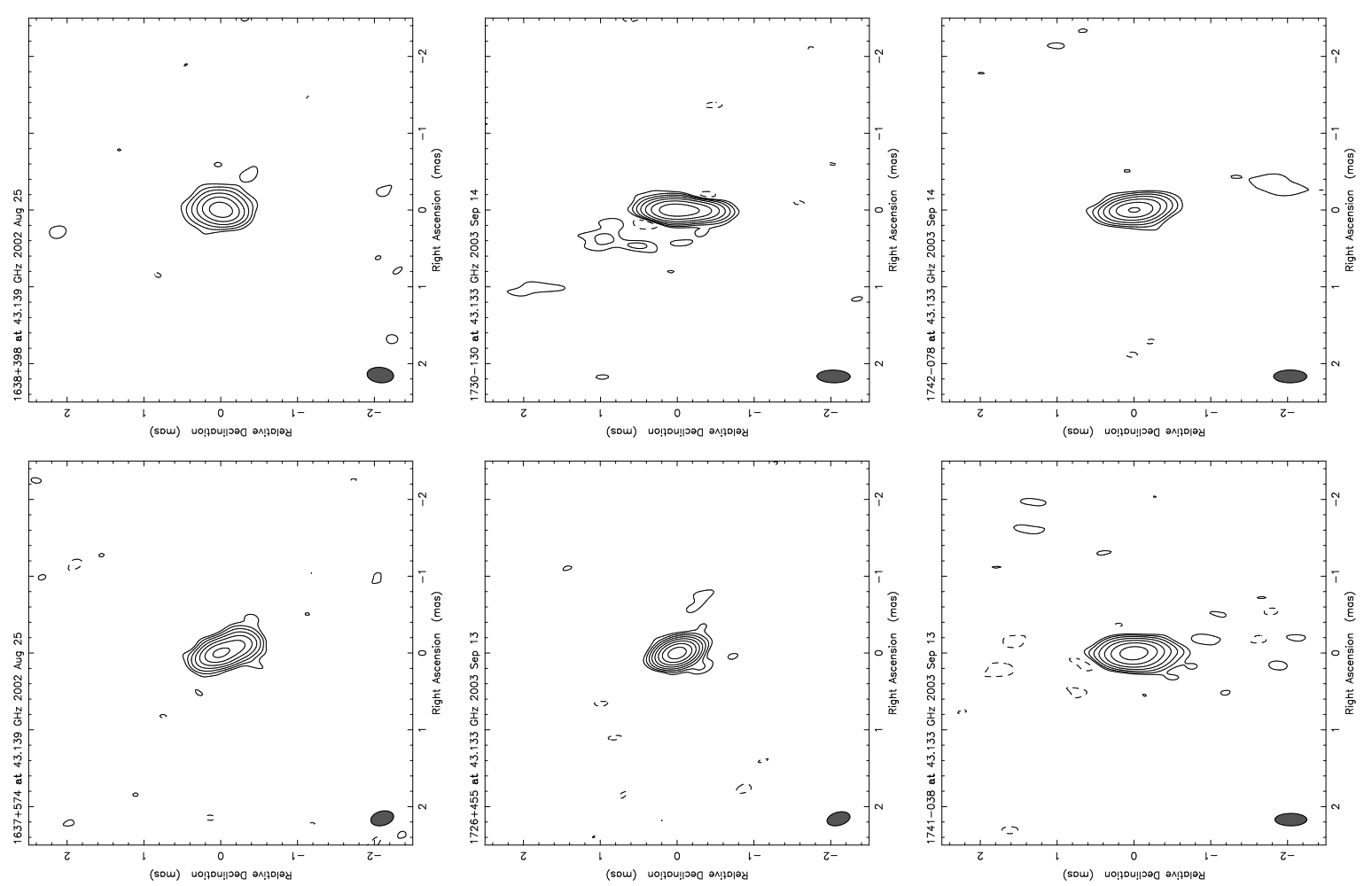

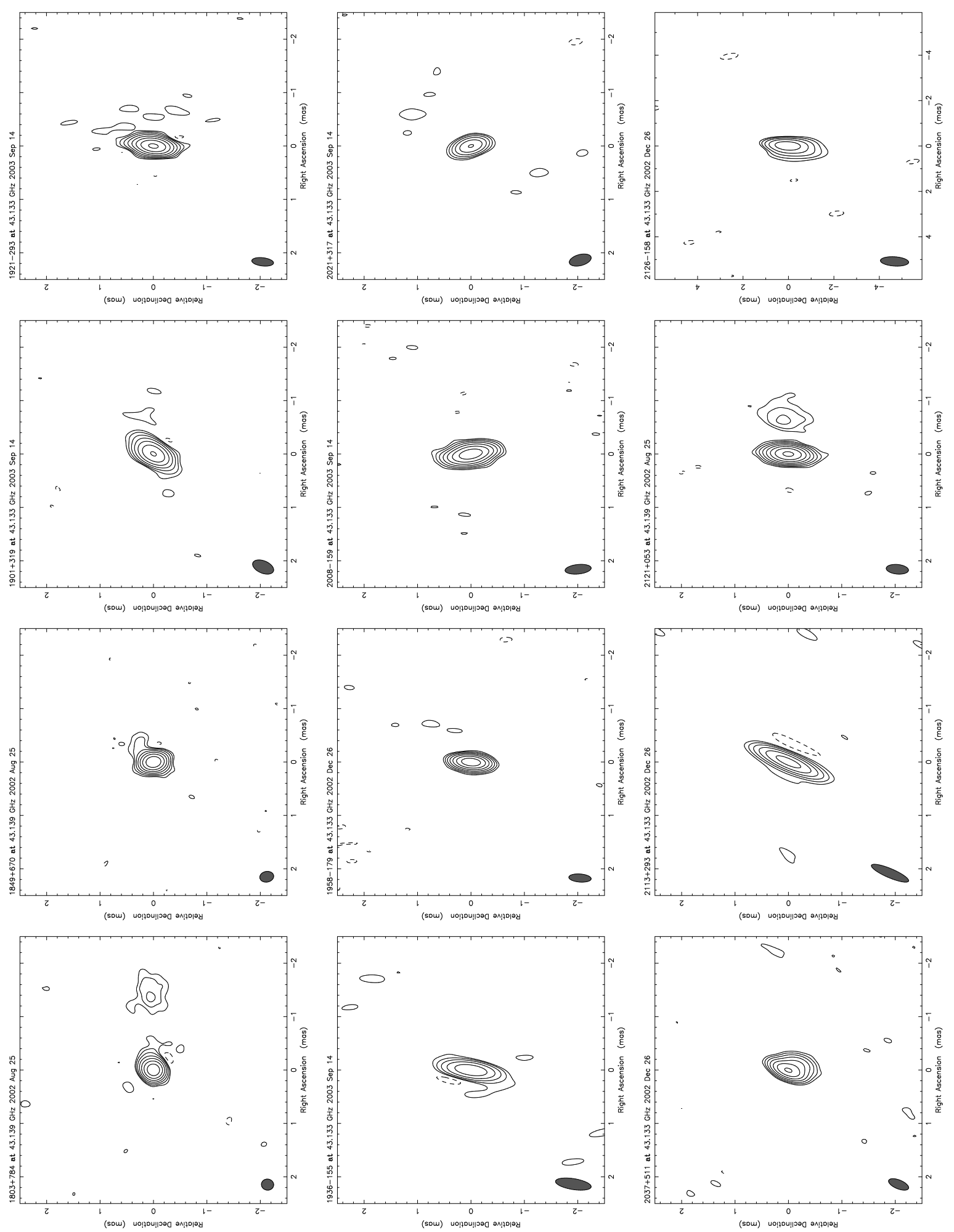

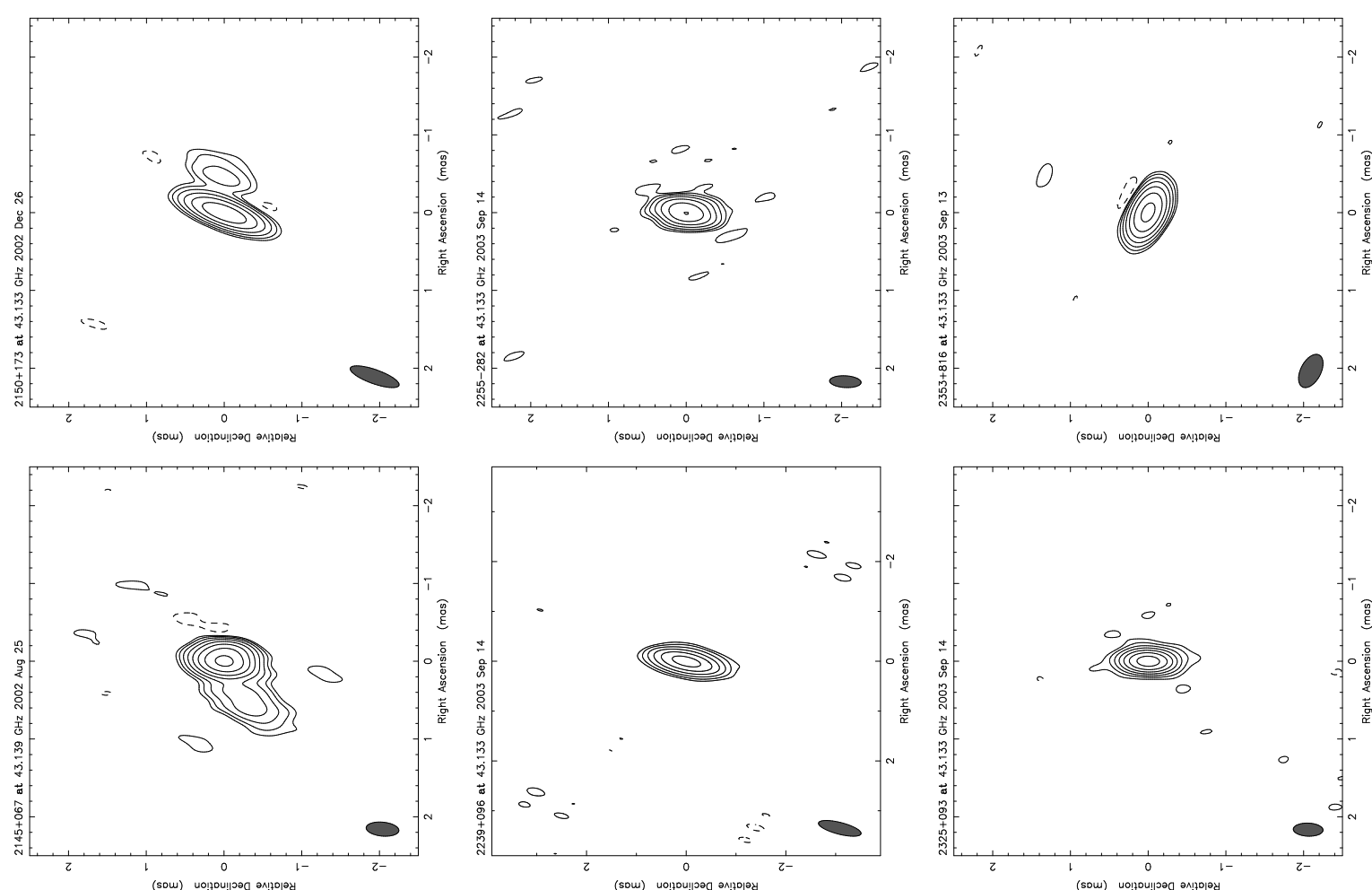

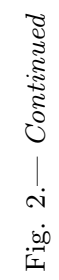
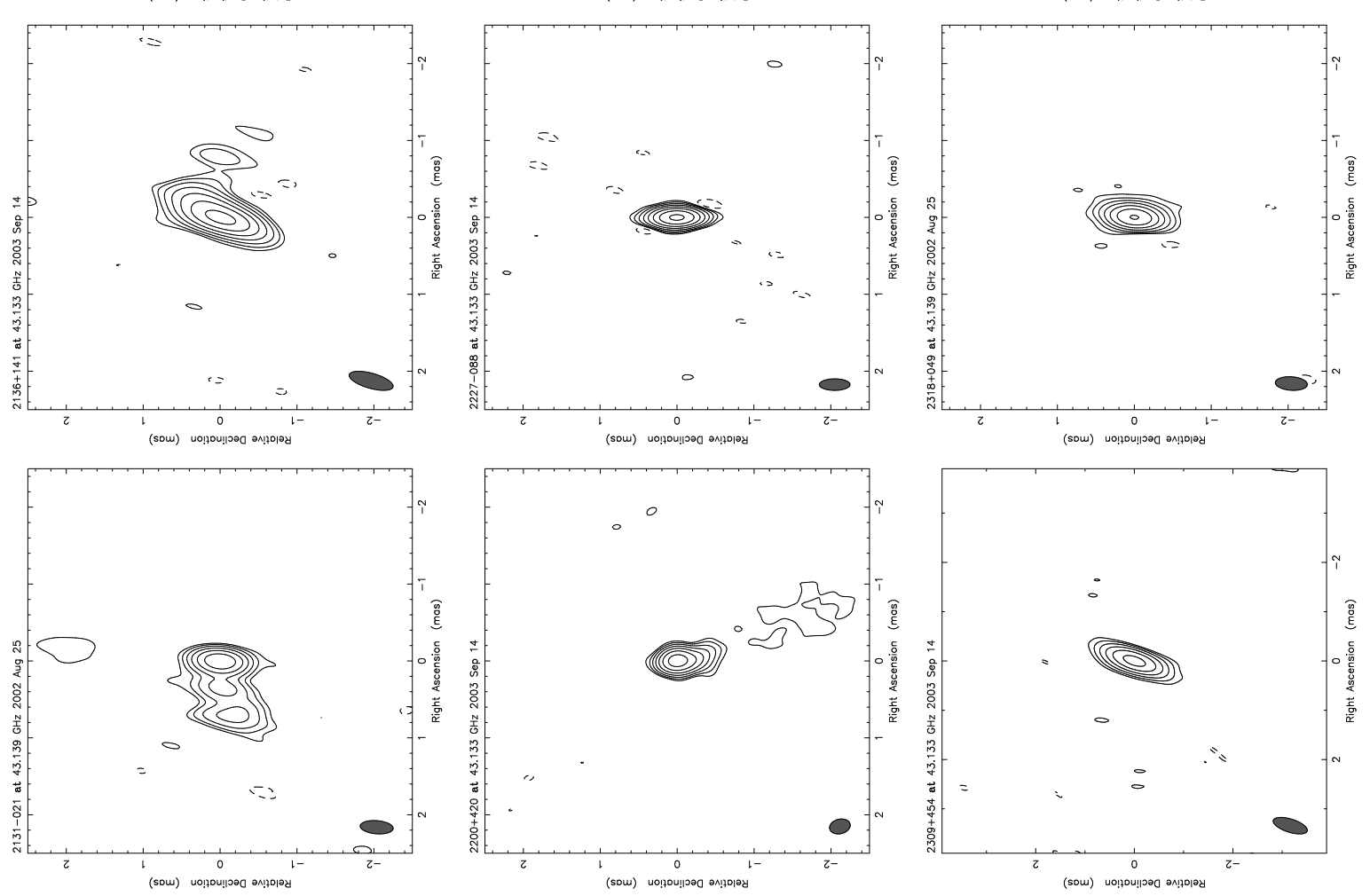

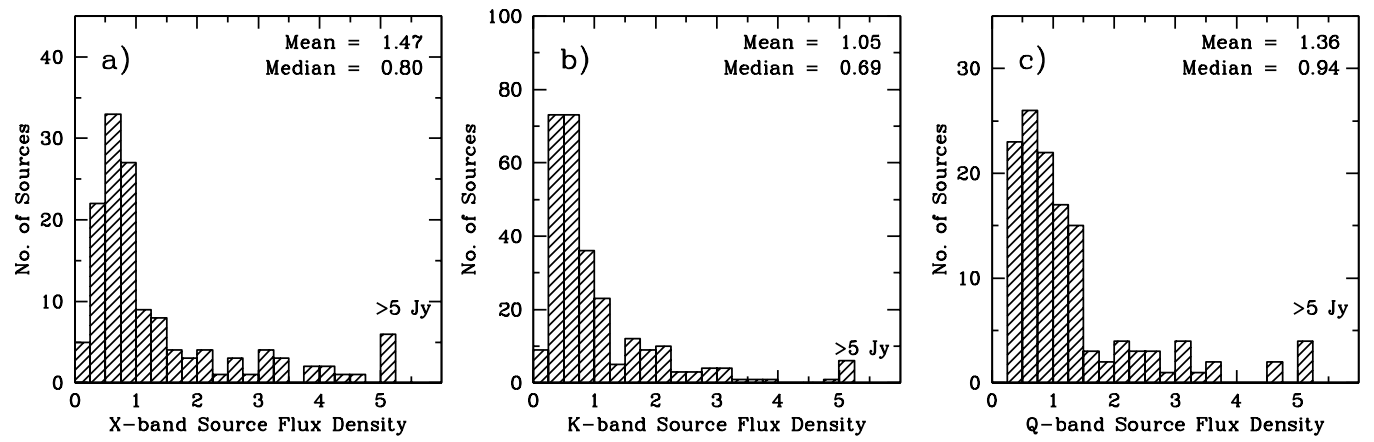

FIG. 3.- Distributions of the mean (averaged over all sessions) source flux density $(\bar{S})$ at a) X band, b) K band, and b) Q band. There were a total of 138,274 , and 132 sources, respectively, for which the flux density was measured at each of the three bands in our VLBA data set.
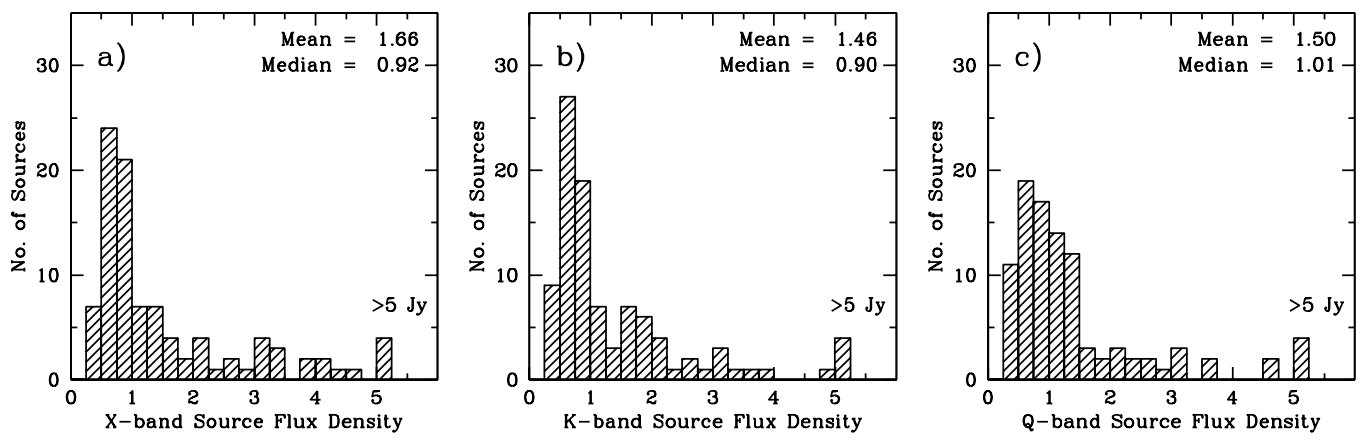

FIG. 4.- Distributions of the mean (averaged over all sessions) source flux density $(\bar{S})$ at a) X band, b) K band, and b) Q band for the 97 sources common to all three frequencies within our VLBA data set.
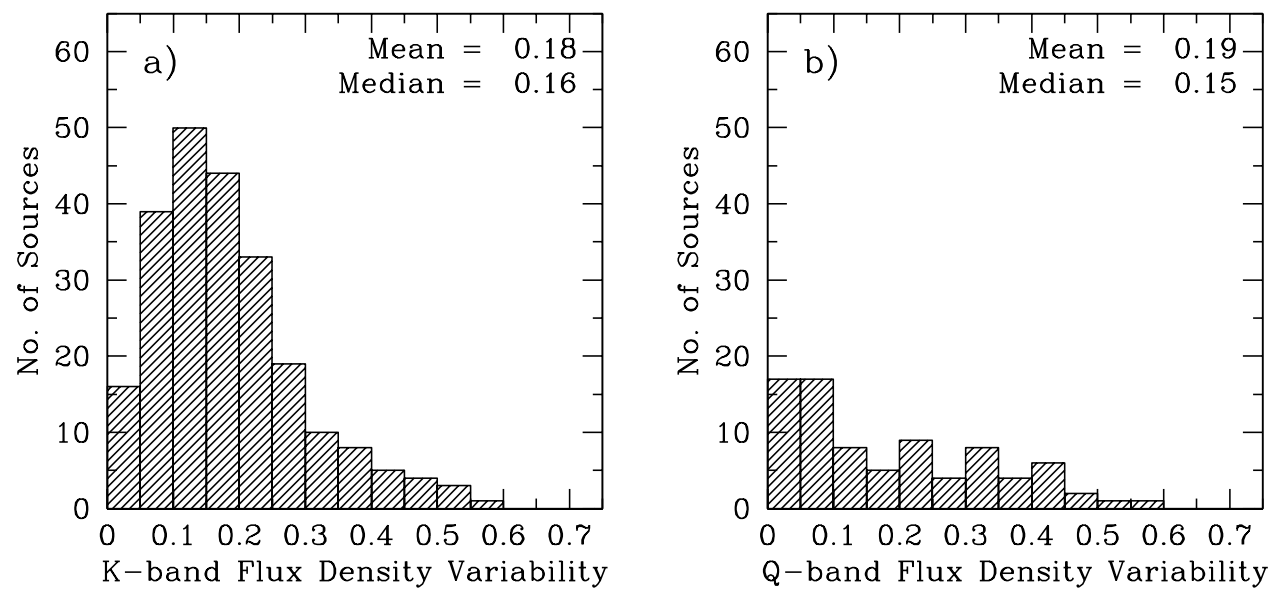

FIG. 5.- Distributions of the source flux density variability index $\left(\sigma_{S} / \bar{S}\right)$ at a) K band and b) Q band. There are a total of 235 sources observed at $\mathrm{K}$ band in more than one session and a total of 82 sources observed at $\mathrm{Q}$ band in more than one session. 

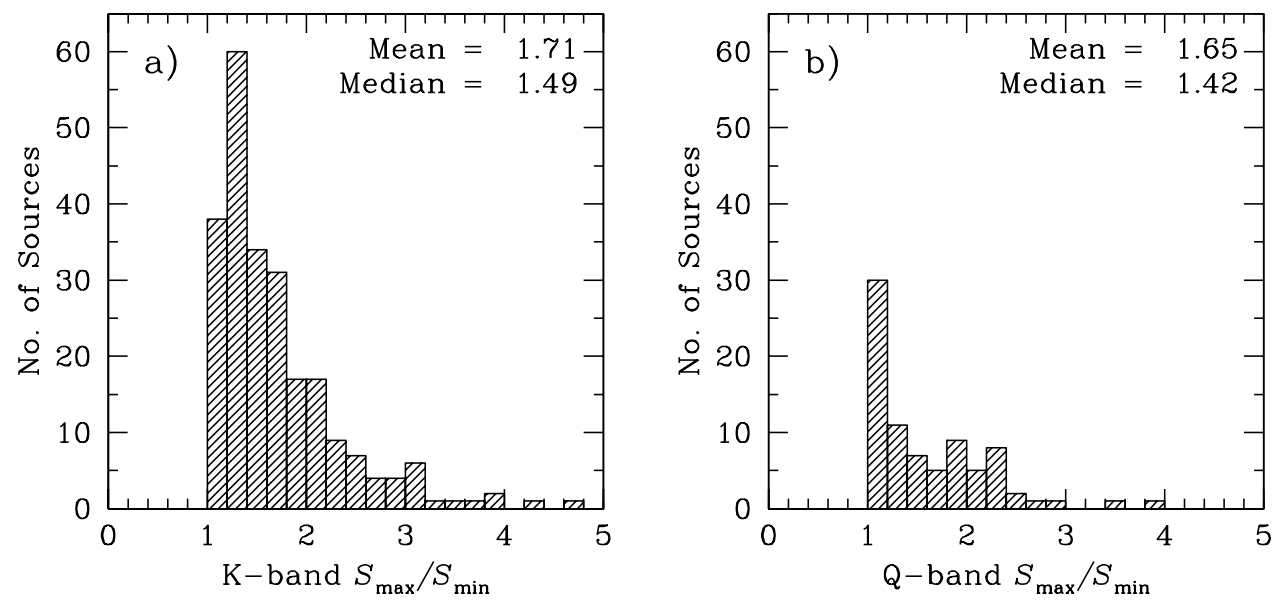

FIG. 6. - Distributions of the ratio of the maximum to minimum source flux density $\left(S_{\max } / S_{\min }\right)$ at a) K band and b) Q band. There are a total of 235 sources observed at $\mathrm{K}$ band in more than one session and a total of 82 sources observed at $\mathrm{Q}$ band in more than one session.
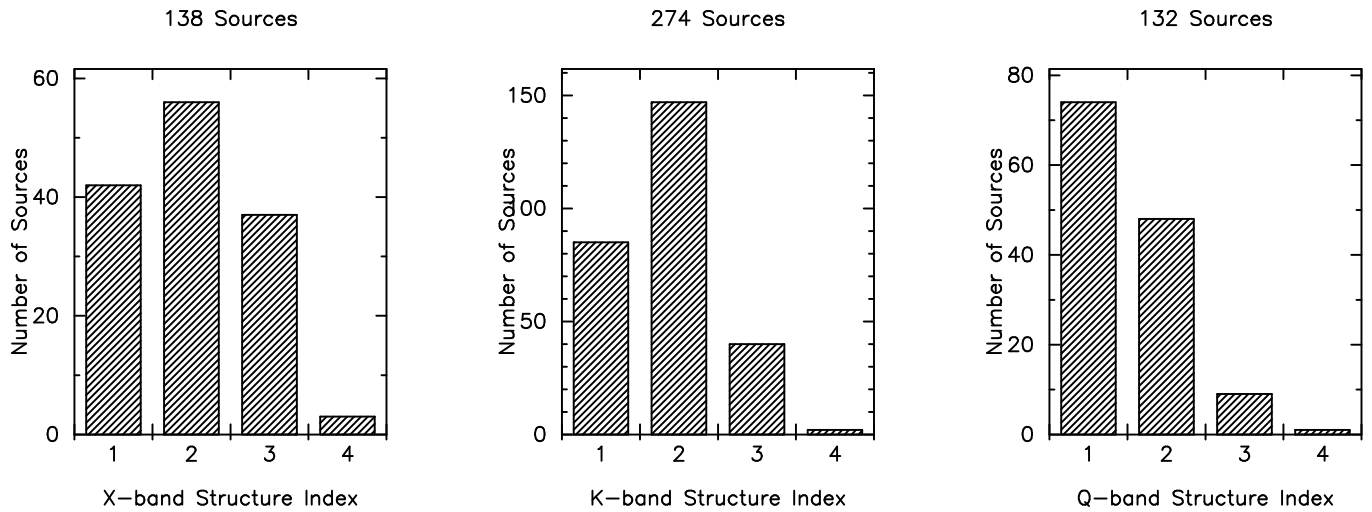

FIG. 7. - Distributions of values for the maximum source structure index $(S I)$ at (a) X band, (b) K band and (c) Q band. There were a total of 138, 274, and 132 sources, respectively, for which the flux density was measured at each of the three bands in our VLBA data set.
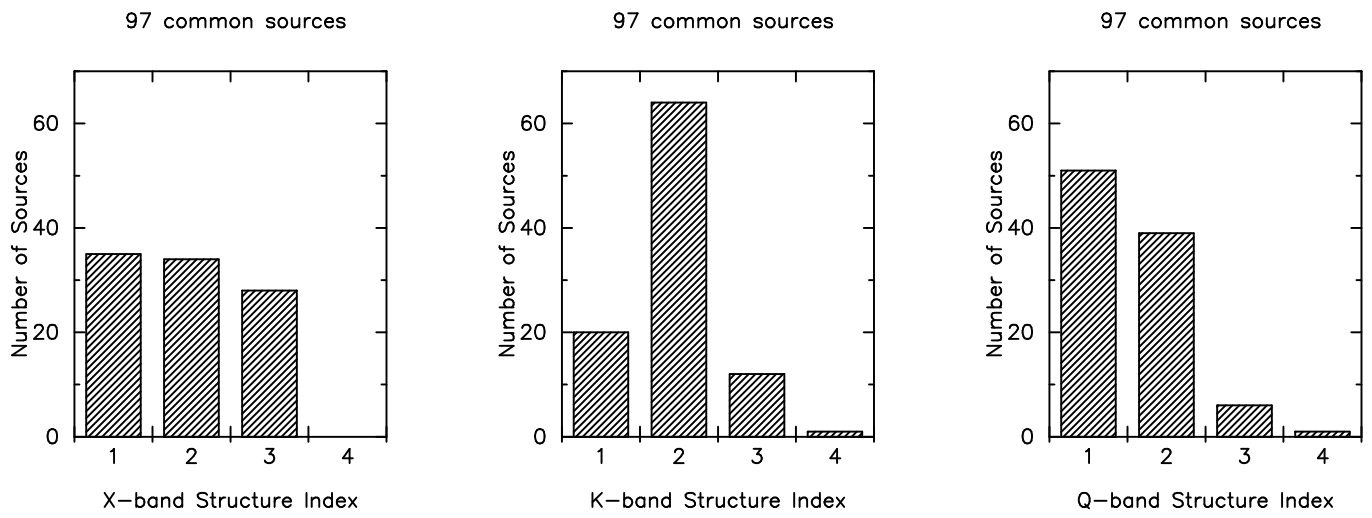

FIG. 8. - Distributions of values for the maximum source structure index (SI) at (a) X band, (b) K band and (c) Q band for the 97 sources common to all three frequencies within our VLBA data set. 

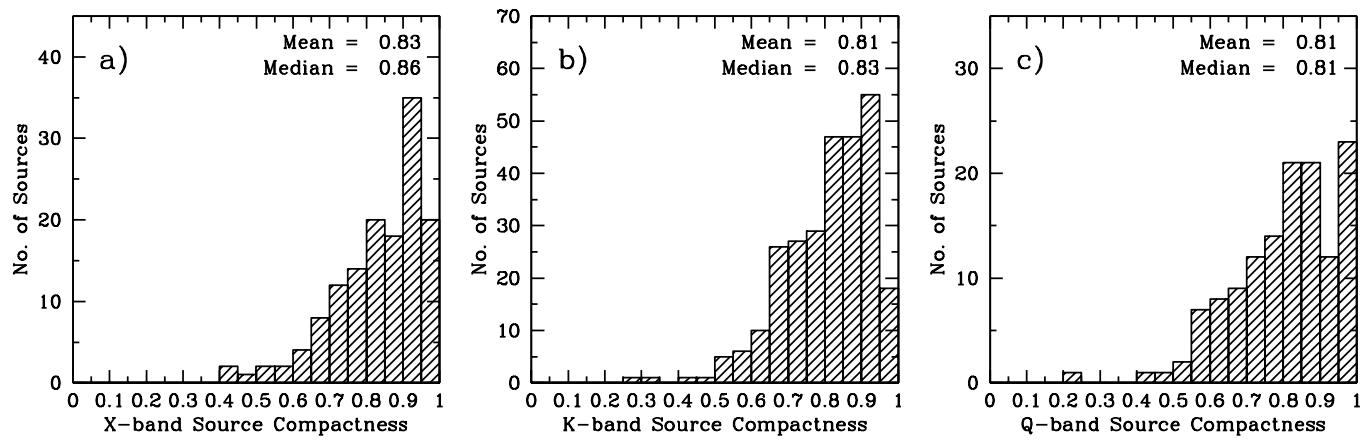

FIG. 9. - Distributions of the mean (averaged over all sessions) source compactness $(\bar{C})$ at (a) X band, (b) K band, and (c) Q band. There were a total of 138,274 , and 132 sources for which the compactness was determined at each of the three bands, respectively. A maximum compactness, $C=1.0$, indicates that all of the flux is contained within one synthesized beam.
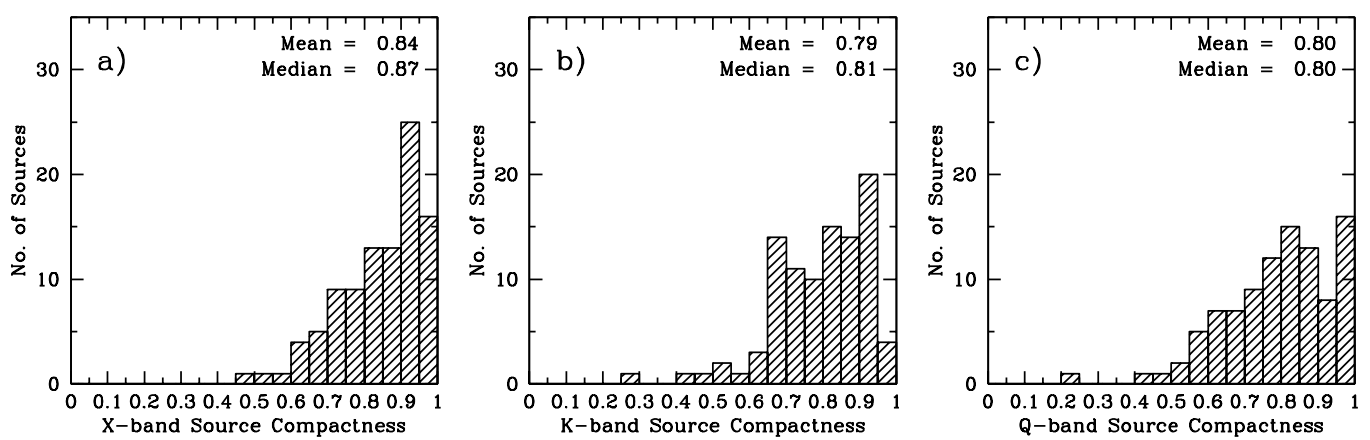

FIG. 10. - Distributions of the mean (averaged over all sessions) source compactness $(\bar{C})$ at (a) X band, (b) K band, and (c) Q band for the 97 sources common to all three frequencies within our VLBA data set. A maximum compactness, $C=1.0$, indicates that all of the flux is contained within one synthesized beam.
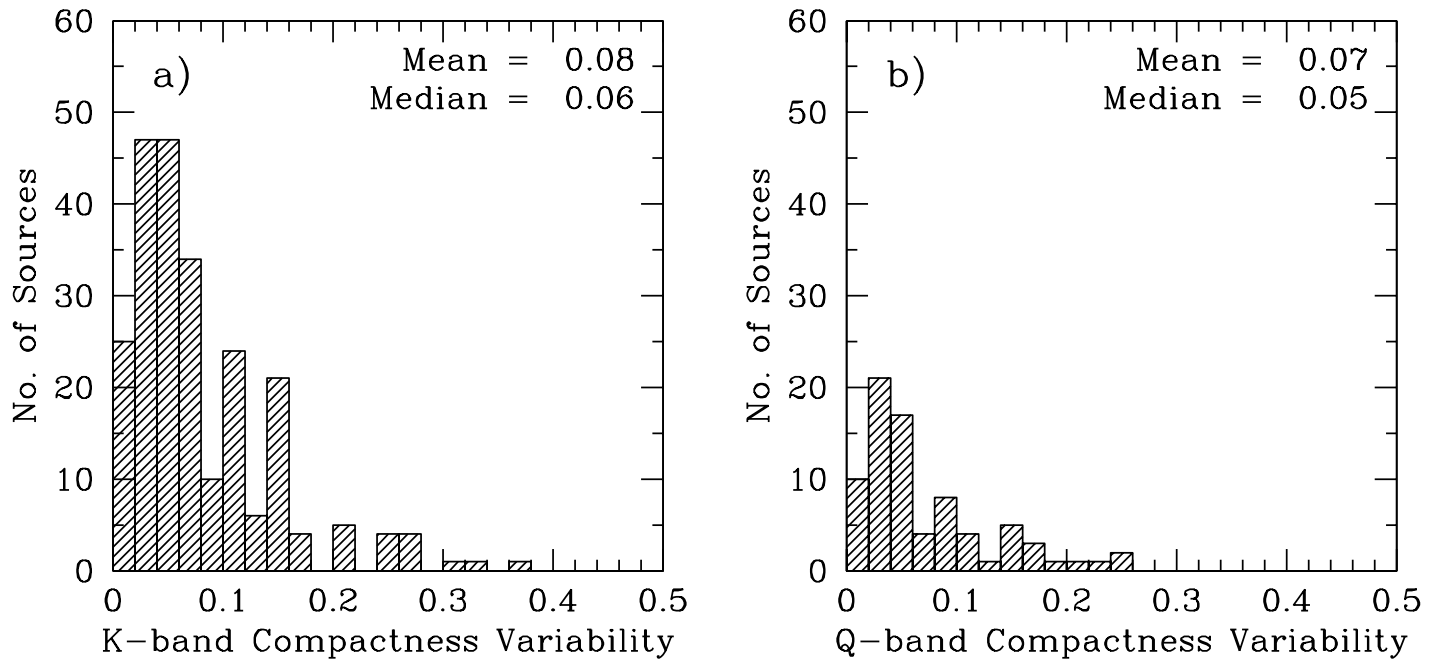

FIG. 11.- Distributions of the source compactness variability index $\left(\sigma_{C} / \bar{C}\right)$ for sources at (a) K band and (b) Q band. There are a total of 235 sources observed in more than one session at $\mathrm{K}$ band, and a total of 82 sources observed in more than one session at Q band. A minimum variability, $\sigma_{C} / \bar{C}=0.0$, indicates no variation in the compactness over time. 

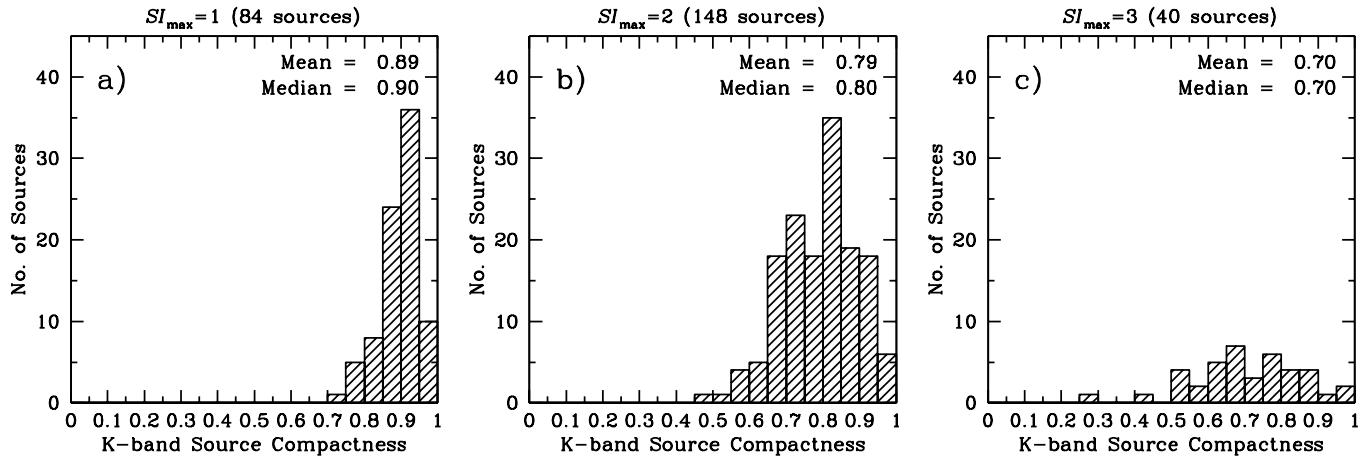

FIG. 12.- Distributions of the mean (averaged over all sessions) source compactness $(\bar{C})$ separated by maximum structure index $\left(S I_{\text {max }}\right)$ for the 274 sources imaged at $\mathrm{K}$ band $(24 \mathrm{GHz})$. The three panels represent sources with (a) $S I_{\max }=1,(\mathrm{~b}) S I_{\max }=2$, and (c) $S I_{\max }=3$, respectively. There are two sources with a $S I_{\max }=4$ that are not shown.
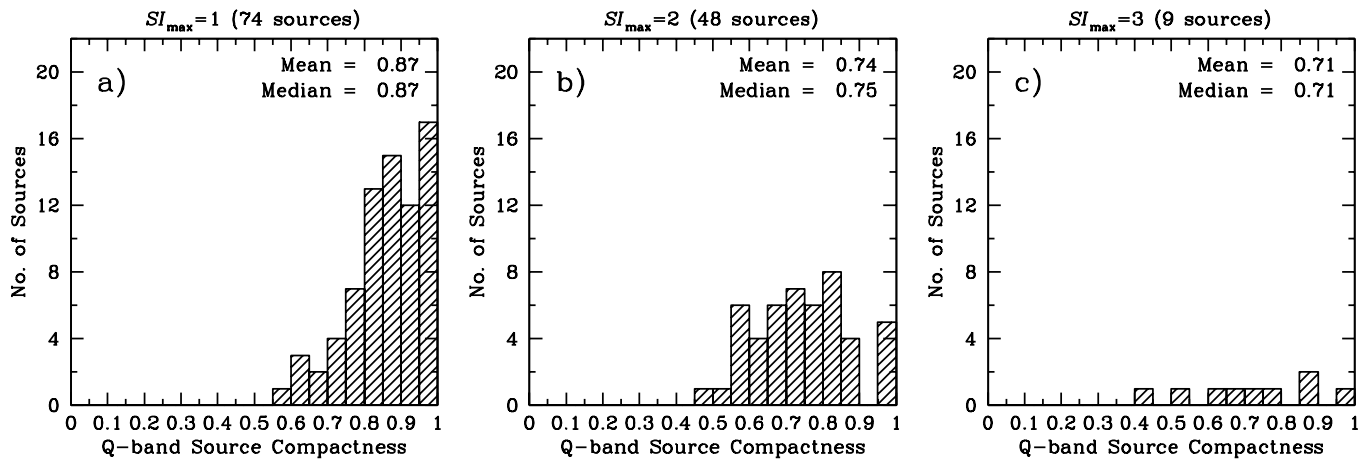

Fig. 13. - Distributions of the mean (averaged over all sessions) source compactness $(\bar{C})$ separated by maximum structure index $\left(S I_{\text {max }}\right)$ for the 132 sources imaged at Q band $(43 \mathrm{GHz})$. The three panels represent sources with (a) $S I_{\max }=1,(\mathrm{~b}) S I_{\max }=2$, and (c) $S I_{\max }=3$, respectively. There is one source with a $S I_{\max }=4$ that is not shown.
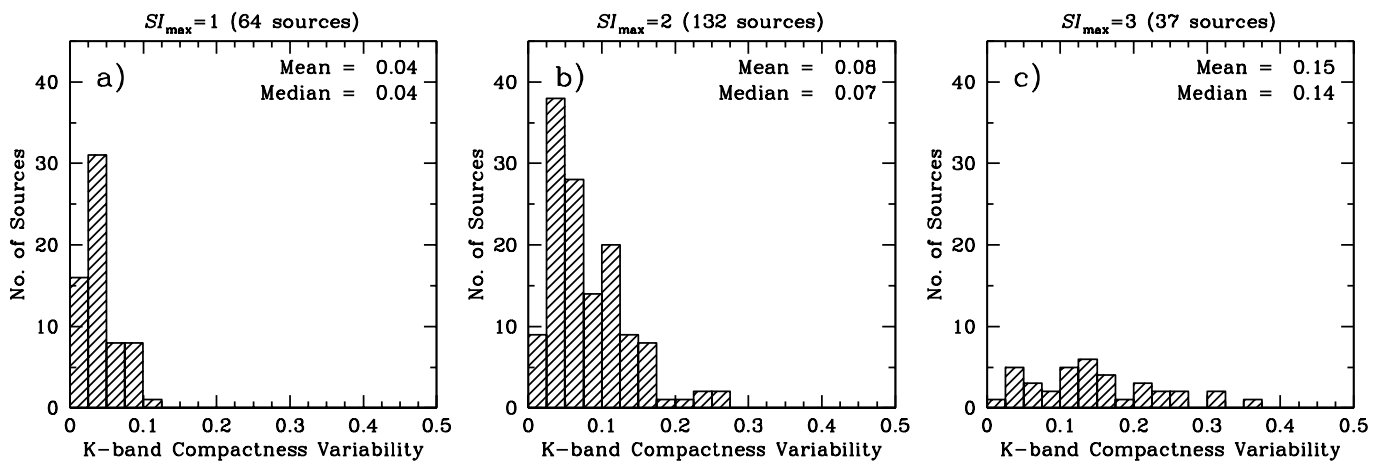

FIG. 14.- Distributions of the compactness variability index $\left(\sigma_{C} / \bar{C}\right)$ separated by maximum structure index $\left(S I_{\max }\right)$ for the 235 sources K-band sources imaged in more than one session. The three panels represent sources with (a) $S I_{\max }=1$, (b) $S I_{\max }=2$, and (c) $S I_{\max }=3$, respectively. There are two sources with a $S I_{\max }=4$ that are not shown. 

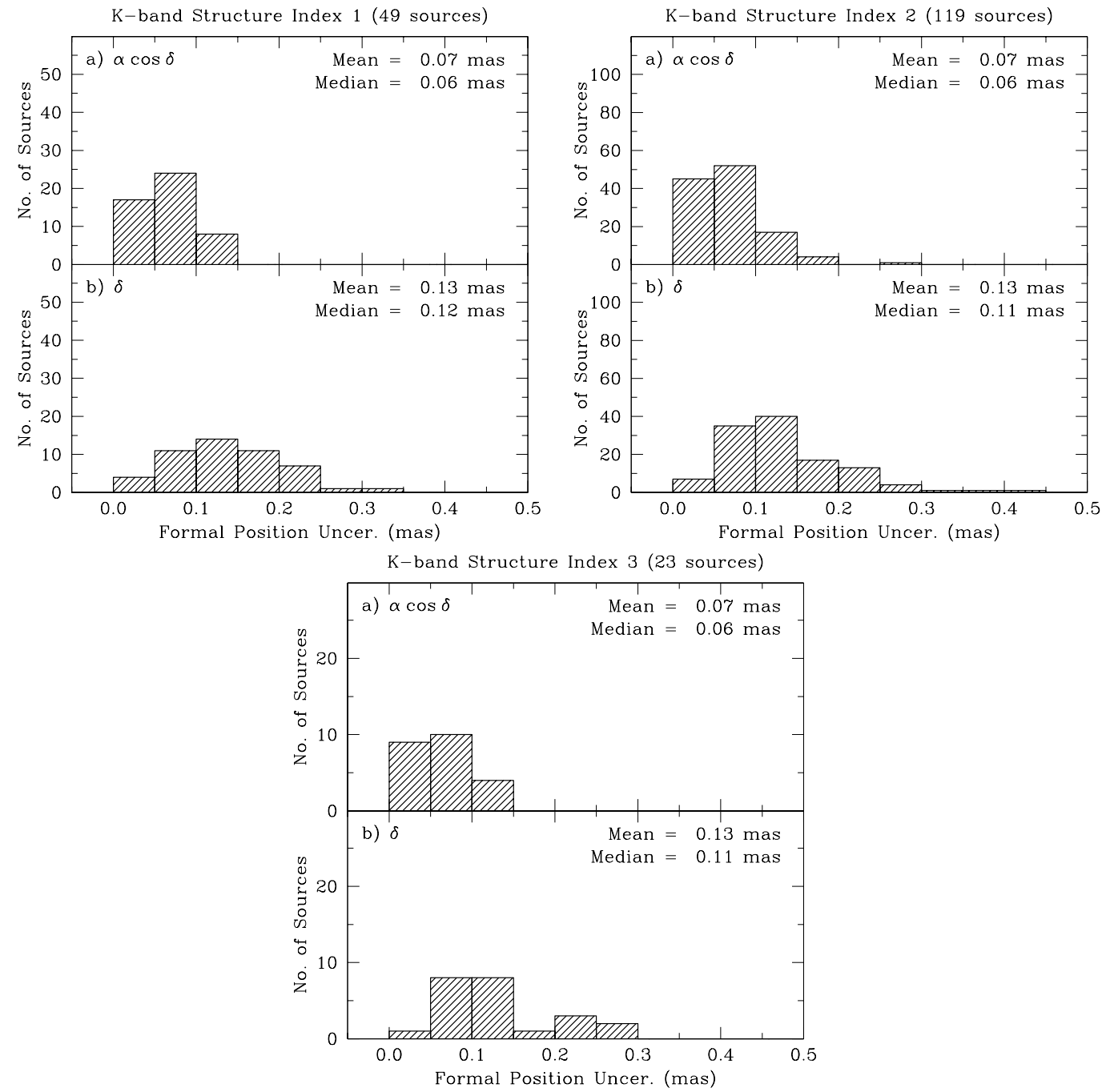

FIG. 15.- Distributions of the formal position uncertainties in $\alpha \cos \delta$ (a) and in $\delta$ (b) for the 193 sources with 100 or more delay observations at $\mathrm{K}$ band $(24 \mathrm{GHz})$. The three panels represent sources with a maximum source structure index equal to 1,2 and 3 , respectively. There are two sources with a structure index of 4 and with more that 100 delay observations that are not included in the figure. 


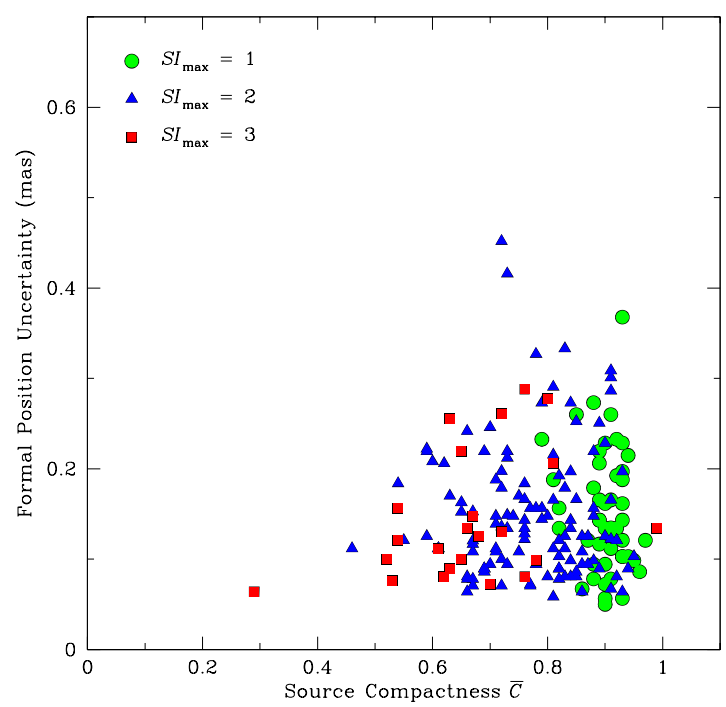

FIG. 16. - Comparison of the formal astrometric position uncertainty versus the mean source compactness $(\bar{C})$ for K band sources with more than 100 delay measurements. Point color and type represent the maximum structure index for each source over the sessions in which it was observed.
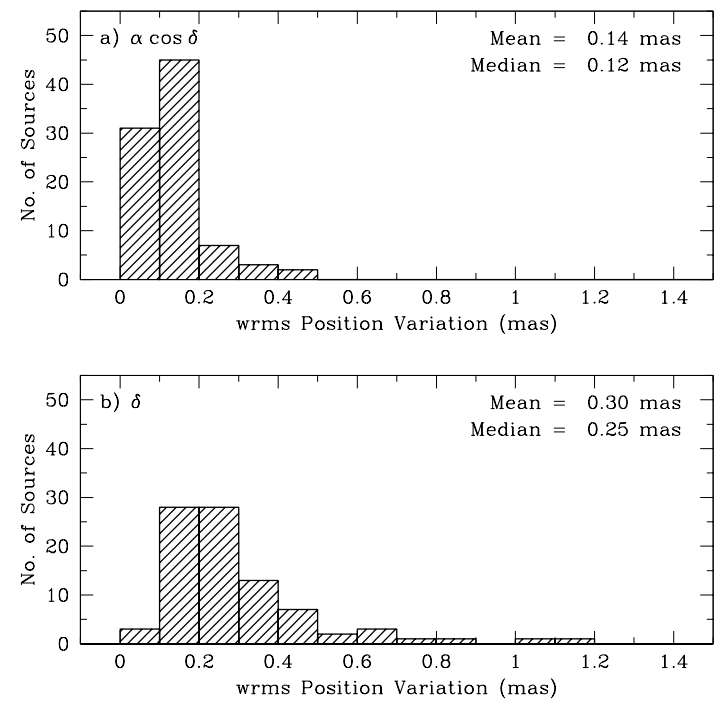

FIG. 17.- Distributions of wrms position stability in (a) $\alpha \cos \delta$ and in (b) $\delta$ for the 88 sources observed in five or more sessions at K-band $(24 \mathrm{GHz})$. 


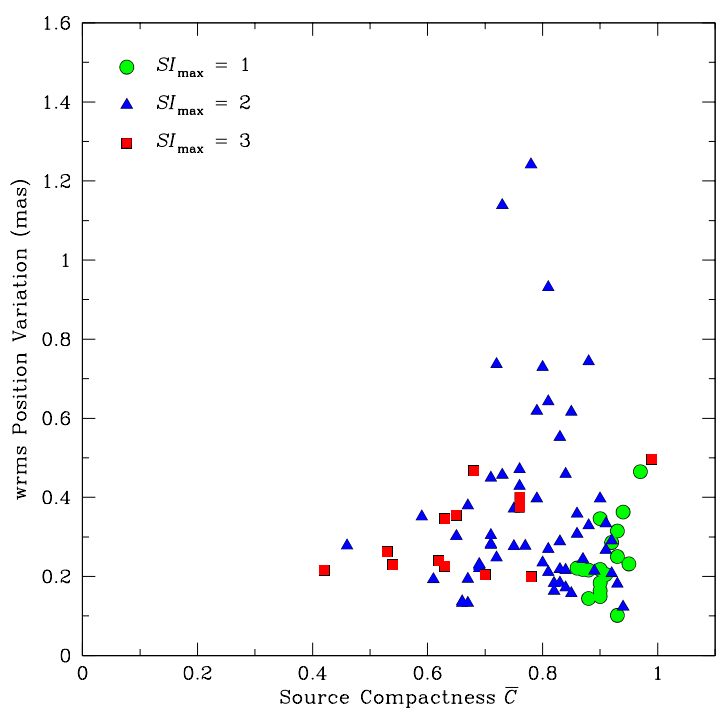

FIG. 18. - Comparison of the wrms position stability versus the mean source compactness $(\bar{C})$ for sources observed in five or more sessions at $\mathrm{K}$ band $(24 \mathrm{GHz})$. Point color and type represent the maximum structure index for each source over the sessions in which it was observed. 
TABLE 1

Summary of VLBA Imaging at K and Q Band.

\begin{tabular}{|c|c|c|c|c|c|c|c|}
\hline \multirow[b]{2}{*}{ Epoch } & \multirow[b]{2}{*}{ Band } & \multicolumn{3}{|c|}{ K-band Sources } & \multicolumn{3}{|c|}{ Q-band Sources } \\
\hline & & Imaged & Overlap & New & Imaged & Overlap & New \\
\hline $2002-05-15$ & $\mathrm{~K}^{\mathrm{a}} / \mathrm{Q}^{\mathrm{b}}$ & 65 & $\cdots$ & 65 & 65 & $\cdots$ & 65 \\
\hline $2002-08-25$ & $\mathrm{~K}^{\mathrm{a}} / \mathrm{Q}^{\mathrm{b}}$ & 65 & 65 & $\ldots$ & 65 & 65 & $\ldots$ \\
\hline $2002-12-26$ & $\mathrm{~K}^{\mathrm{c}} / \mathrm{Q}^{\mathrm{d}}$ & 67 & 24 & 43 & 67 & 24 & 43 \\
\hline $2003-05-22^{\mathrm{e}}$ & $\mathrm{K}^{\mathrm{c}}$ & 184 & 62 & 122 & $\cdots$ & $\cdots$ & $\cdots$ \\
\hline 2003-09-13 & $\mathrm{K}^{\mathrm{c}} / \mathrm{Q}^{\mathrm{d}}$ & 70 & 70 & $\ldots$ & 70 & 46 & 24 \\
\hline 2004-02-15 & $\mathrm{K}^{\mathrm{c}}$ & 65 & 65 & $\cdots$ & $\cdots$ & $\cdots$ & $\cdots$ \\
\hline 2004-12-14 & $K^{f}$ & 101 & 101 & $\ldots$ & $\ldots$ & $\ldots$ & $\ldots$ \\
\hline $2005-08-26$ & $K^{f}$ & 92 & 67 & 25 & $\ldots$ & $\ldots$ & $\ldots$ \\
\hline 2006-07-09 & $K^{f}$ & 178 & 163 & 15 & $\ldots$ & $\ldots$ & $\ldots$ \\
\hline 2007-03-30 & $K^{f}$ & 185 & 181 & 4 & $\ldots$ & $\ldots$ & $\ldots$ \\
\hline
\end{tabular}

${ }^{\mathrm{a}} \nu=24.25,24.33,24.51,24.65 \mathrm{GHz}$

b $\nu=42.95,43.03,43.21,43.35 \mathrm{GHz}$

c $\nu=24.21,24.23,24.27,24.34,24.49,24.59,24.66,24.68 \mathrm{GHz}$

d $\nu=42.91,42.93,42.97,43.04,43.19,43.29,43.36,43.38 \mathrm{GHz}$

e K-band only survey of 249 sources.

f $\nu=23.72,23.74,23.78,23.85,24.00,24.10,24.17,24.19 \mathrm{GHz}$ 
The Celestial Reference Frame at 24 and $43 \mathrm{GHz}$

TABLE 2

Parameters of Naturally Weighted Images at $24 \mathrm{GHz}$

\begin{tabular}{|c|c|c|c|c|c|c|c|c|}
\hline \multirow[b]{2}{*}{ Source } & \multirow[b]{2}{*}{$\begin{array}{l}\text { Observation } \\
\text { Session }\end{array}$} & \multicolumn{3}{|c|}{ Beam $^{\mathrm{a}}$} & \multicolumn{3}{|c|}{ Flux Density } & \multirow[b]{2}{*}{$\begin{array}{l}\text { Contour Levels } \\
\left(\mathrm{mJy} \mathrm{bm}^{-1}\right)\end{array}$} \\
\hline & & $\begin{array}{c}a \\
\text { (mas) }\end{array}$ & $\begin{array}{c}b \\
\text { (mas) }\end{array}$ & $\begin{array}{c}\phi \\
\left({ }^{\circ}\right)\end{array}$ & $\begin{array}{c}S_{\text {peak }} \\
\left(\mathrm{Jy} \mathrm{bm}^{-1}\right)\end{array}$ & $\begin{array}{c}S_{\text {total }^{\mathrm{b}}} \\
\text { (Jy) }\end{array}$ & $\begin{array}{c}S_{\mathrm{rms}} \mathrm{c} \\
\left(\mathrm{mJy} \mathrm{bm}^{-1}\right)\end{array}$ & \\
\hline $0006+061$ & $05-22-2003$ & 1.01 & 0.29 & -11 & 0.24 & 0.26 & 2.9 & $7.1 \times\left(1, \ldots, 2^{5}\right)$ \\
\hline $0007+106$ & $03-30-2007$ & 0.72 & 0.30 & 3 & 0.42 & 0.51 & 1.5 & $4.5 \times\left(1, \ldots, 2^{6}\right)$ \\
\hline $0009+081$ & $03-30-2007$ & 0.82 & 0.37 & -4 & 0.31 & 0.45 & 1.4 & $4.1 \times\left(1, \ldots, 2^{6}\right)$ \\
\hline $0016+731$ & $12-14-2004$ & 0.46 & 0.36 & -2 & 1.82 & 1.98 & 1.4 & $4.1 \times\left(1, \ldots, 2^{8}\right)$ \\
\hline $0017+200$ & $03-30-2007$ & 0.63 & 0.31 & -9 & 0.43 & 0.59 & 1.8 & $5.4 \times\left(1, \ldots, 2^{6}\right)$ \\
\hline $0019+058$ & 03-30-2007 & 0.73 & 0.30 & -3 & 0.87 & 0.94 & 1.3 & $3.9 \times\left(1, \ldots, 2^{7}\right)$ \\
\hline $0038-020$ & $05-22-2003$ & 1.39 & 0.70 & -1 & 0.29 & 0.35 & 3.6 & $10.9 \times\left(1, \ldots, 2^{4}\right)$ \\
\hline $0046+316$ & $03-30-2007$ & 0.63 & 0.34 & 7 & 0.39 & 0.53 & 1.4 & $3.9 \times\left(1, \ldots, 2^{6}\right)$ \\
\hline $0048-097$ & $12-14-2004$ & 0.77 & 0.31 & 1 & 0.74 & 0.78 & 1.5 & $4.4 \times\left(1, \ldots, 2^{7}\right)$ \\
\hline $0054+161$ & $05-22-2003$ & 1.12 & 0.32 & -10 & 0.25 & 0.26 & 2.9 & $8.2 \times\left(1, \ldots, 2^{4}\right)$ \\
\hline $0059+581$ & $12-14-2004$ & 0.66 & 0.33 & 30 & 1.31 & 1.60 & 1.2 & $3.7 \times\left(1, \ldots, 2^{8}\right)$ \\
\hline $0104-408$ & $05-22-2003$ & 0.74 & 0.26 & -3 & 0.85 & 1.08 & 5.0 & $15.0 \times\left(1, \ldots, 2^{5}\right)$ \\
\hline $0109+224$ & 03-30-2007 & 0.64 & 0.36 & -5 & 0.85 & 0.95 & 1.5 & $4.4 \times\left(1, \ldots, 2^{7}\right)$ \\
\hline $0110+495$ & $03-30-2007$ & 0.73 & 0.30 & 23 & 1.16 & 1.35 & 1.9 & $5.5 \times\left(1, \ldots, 2^{7}\right)$ \\
\hline $0111+021$ & $03-30-2007$ & 1.07 & 0.33 & -15 & 0.33 & 0.48 & 1.8 & $5.4 \times\left(1, \ldots, 2^{5}\right)$ \\
\hline $0119+041$ & $03-30-2007$ & 0.98 & 0.36 & -13 & 0.44 & 0.67 & 4.2 & $12.5 \times\left(1, \ldots, 2^{5}\right)$ \\
\hline $0119+115$ & $12-14-2004$ & 0.71 & 0.32 & -4 & 0.99 & 1.26 & 1.3 & $4.0 \times\left(1, \ldots, 2^{7}\right)$ \\
\hline $0119+247$ & $03-30-2007$ & 0.71 & 0.36 & -5 & 0.26 & 0.47 & 1.1 & $3.4 \times\left(1, \ldots, 2^{6}\right)$ \\
\hline $0122-003$ & 03-30-2007 & 0.75 & 0.30 & -1 & 0.42 & 0.68 & 3.1 & $9.4 \times\left(1, \ldots, 2^{5}\right)$ \\
\hline $0123+257$ & $03-30-2007$ & 0.82 & 0.31 & -6 & 0.40 & 0.46 & 1.6 & $4.7 \times\left(1, \ldots, 2^{6}\right)$ \\
\hline $0127+084$ & 03-30-2007 & 1.05 & 0.33 & -13 & 0.26 & 0.27 & 1.3 & $3.9 \times\left(1, \ldots, 2^{6}\right)$ \\
\hline $0133+476$ & $12-14-2004$ & 0.54 & 0.33 & 12 & 2.82 & 3.16 & 2.2 & $6.6 \times\left(1, \ldots, 2^{8}\right)$ \\
\hline $0138-097$ & $12-14-2004$ & 0.89 & 0.34 & -8 & 0.21 & 0.33 & 1.2 & $3.6 \times\left(1, \ldots, 2^{5}\right)$ \\
\hline $0149+218$ & $03-30-2007$ & 0.67 & 0.33 & -2 & 0.71 & 0.81 & 1.9 & $5.6 \times\left(1, \ldots, 2^{6}\right)$ \\
\hline $0202+149$ & $03-30-2007$ & 0.71 & 0.32 & -8 & 0.40 & 0.74 & 2.2 & $6.7 \times\left(1, \ldots, 2^{5}\right)$ \\
\hline $0202+319$ & 03-30-2007 & 0.73 & 0.30 & 15 & 2.65 & 2.89 & 2.9 & $8.7 \times\left(1, \ldots, 2^{8}\right)$ \\
\hline $0212+735$ & $03-30-2007$ & 0.45 & 0.35 & 20 & 1.12 & 2.87 & 3.4 & $10.2 \times\left(1, \ldots, 2^{6}\right)$ \\
\hline $0213-026$ & $03-30-2007$ & 0.80 & 0.34 & 0 & 0.28 & 0.42 & 2.0 & $5.9 \times\left(1, \ldots, 2^{5}\right)$ \\
\hline $0220-349$ & $05-22-2003$ & 5.43 & 1.04 & 8 & 0.48 & 0.55 & 3.8 & $11.5 \times\left(1, \ldots, 2^{5}\right)$ \\
\hline $0221+067$ & $03-30-2007$ & 0.83 & 0.36 & -11 & 0.43 & 0.57 & 1.4 & $4.3 \times\left(1, \ldots, 2^{6}\right)$ \\
\hline $0222+185$ & $03-30-2007$ & 0.92 & 0.36 & -19 & 0.21 & 0.21 & 1.4 & $4.2 \times\left(1, \ldots, 2^{5}\right)$ \\
\hline $0224+671$ & $12-14-2004$ & 0.57 & 0.36 & 41 & 0.53 & 0.70 & 1.2 & $3.7 \times\left(1, \ldots, 2^{7}\right)$ \\
\hline $0229+131$ & $12-14-2004$ & 0.74 & 0.33 & -2 & 0.53 & 0.85 & 3.1 & $9.2 \times\left(1, \ldots, 2^{5}\right)$ \\
\hline $0234+285$ & $03-30-2007$ & 0.63 & 0.32 & 3 & 1.07 & 2.34 & 3.8 & $11.4 \times\left(1, \ldots, 2^{6}\right)$ \\
\hline $0235+164$ & $03-30-2007$ & 0.69 & 0.31 & -5 & 3.74 & 3.89 & 3.4 & $10.1 \times\left(1, \ldots, 2^{8}\right)$ \\
\hline $0237-027$ & $03-30-2007$ & 0.70 & 0.28 & -3 & 0.61 & 0.63 & 1.9 & $5.2 \times\left(1, \ldots, 2^{6}\right)$ \\
\hline $0237+040$ & $03-30-2007$ & 0.76 & 0.31 & -5 & 0.53 & 0.59 & 1.9 & $5.8 \times\left(1, \ldots, 2^{6}\right)$ \\
\hline $0239+108$ & $03-30-2007$ & 0.73 & 0.31 & -6 & 0.53 & 0.61 & 1.4 & $4.3 \times\left(1, \ldots, 2^{6}\right)$ \\
\hline $0241+622$ & $12-14-2004$ & 0.72 & 0.31 & 33 & 0.57 & 1.05 & 1.7 & $5.2 \times\left(1, \ldots, 2^{6}\right)$ \\
\hline $0250+320$ & $05-22-2003$ & 0.79 & 0.27 & -1 & 0.23 & 0.21 & 1.5 & $4.6 \times\left(1, \ldots, 2^{5}\right)$ \\
\hline $0256+192$ & $03-30-2007$ & 1.13 & 0.37 & -9 & 0.26 & 0.29 & 1.7 & $5.1 \times\left(1, \ldots, 2^{5}\right)$ \\
\hline $0300+470$ & $03-30-2007$ & 0.71 & 0.29 & 5 & 0.55 & 0.70 & 1.4 & $4.2 \times\left(1, \ldots, 2^{7}\right)$ \\
\hline $0306+102$ & $12-14-2004$ & 0.82 & 0.33 & 3 & 1.09 & 1.25 & 2.0 & $5.9 \times\left(1, \ldots, 2^{7}\right)$ \\
\hline $0309+411$ & $03-30-2007$ & 0.70 & 0.30 & 13 & 0.78 & 1.01 & 1.5 & $4.6 \times\left(1, \ldots, 2^{7}\right)$ \\
\hline $0322+222$ & $03-30-2007$ & 0.71 & 0.30 & -5 & 0.42 & 0.49 & 1.3 & $3.9 \times\left(1, \ldots, 2^{6}\right)$ \\
\hline $0333+321$ & $12-14-2004$ & 0.67 & 0.31 & 1 & 1.63 & 2.01 & 2.4 & $7.0 \times\left(1, \ldots, 2^{7}\right)$ \\
\hline $0336-019$ & $12-14-2004$ & 0.52 & 0.26 & -21 & 0.67 & 1.49 & 2.6 & $7.9 \times\left(1, \ldots, 2^{6}\right)$ \\
\hline $0340+362$ & $03-30-2007$ & 0.65 & 0.34 & -2 & 0.28 & 0.34 & 1.2 & $3.4 \times\left(1, \ldots, 2^{6}\right)$ \\
\hline $0345+460$ & 03-30-2007 & 0.67 & 0.37 & -9 & 0.21 & 0.30 & 1.0 & $2.9 \times\left(1, \ldots, 2^{6}\right)$ \\
\hline $0346-279$ & 03-30-2007 & 1.02 & 0.37 & 5 & 0.49 & 0.71 & 4.4 & $13.3 \times\left(1, \ldots, 2^{5}\right)$ \\
\hline $0350+465$ & $03-30-2007$ & 0.56 & 0.31 & 4 & 0.34 & 0.40 & 1.1 & $3.4 \times\left(1, \ldots, 2^{6}\right)$ \\
\hline $0354+231$ & $03-30-2007$ & 0.75 & 0.31 & 3 & 0.22 & 0.23 & 1.1 & $3.4 \times\left(1, \ldots, 2^{6}\right)$ \\
\hline $0358+040$ & 03-30-2007 & 0.73 & 0.32 & -1 & 0.45 & 0.44 & 2.0 & $5.9 \times\left(1, \ldots, 2^{6}\right)$ \\
\hline $0358+210$ & $03-30-2007$ & 0.78 & 0.30 & -1 & 0.21 & 0.23 & 1.2 & $3.4 \times\left(1, \ldots, 2^{5}\right)$ \\
\hline $0400+258$ & $03-30-2007$ & 0.76 & 0.33 & -9 & 0.42 & 0.77 & 1.9 & $5.7 \times\left(1, \ldots, 2^{6}\right)$ \\
\hline $0402-362$ & $05-22-2003$ & 0.74 & 0.27 & 5 & 2.02 & 2.14 & 4.0 & $12.7 \times\left(1, \ldots, 2^{7}\right)$ \\
\hline $0405-331$ & $03-30-2007$ & 1.10 & 0.36 & 7 & 0.61 & 0.65 & 9.2 & $21.2 \times\left(1, \ldots, 2^{4}\right)$ \\
\hline $0405-385$ & $05-22-2003$ & 0.74 & 0.27 & 1 & 0.54 & 0.69 & 4.0 & $10.8 \times\left(1, \ldots, 2^{5}\right)$ \\
\hline $0406+121$ & $05-22-2003$ & 1.95 & 0.34 & -23 & 0.23 & 0.27 & 2.8 & $8.3 \times\left(1, \ldots, 2^{4}\right)$ \\
\hline $0409+229$ & $03-30-2007$ & 0.88 & 0.54 & -24 & 0.19 & 0.25 & 1.6 & $4.7 \times\left(1, \ldots, 2^{5}\right)$ \\
\hline $0415+398$ & 03-30-2007 & 0.75 & 0.36 & 14 & 0.25 & 0.32 & 1.3 & $3.9 \times\left(1, \ldots, 2^{5}\right)$ \\
\hline $0420-014$ & $03-30-2007$ & 0.74 & 0.28 & -2 & 4.24 & 4.72 & 6.4 & $19.1 \times\left(1, \ldots, 2^{7}\right)$ \\
\hline $0420+022$ & $03-30-2007$ & 0.73 & 0.28 & -2 & 0.66 & 0.91 & 2.1 & $6.3 \times\left(1, \ldots, 2^{6}\right)$ \\
\hline $0422+004$ & $03-30-2007$ & 0.72 & 0.28 & -2 & 0.85 & 0.98 & 1.9 & $5.8 \times\left(1, \ldots, 2^{7}\right)$ \\
\hline $0422-380$ & $05-22-2003$ & 0.73 & 0.27 & 2 & 0.64 & 1.04 & 4.7 & $14.2 \times\left(1, \ldots, 2^{5}\right)$ \\
\hline $0425+048$ & $05-22-2003$ & 1.16 & 0.52 & -17 & 0.24 & 0.30 & 2.5 & $7.6 \times\left(1, \ldots, 2^{4}\right)$ \\
\hline $0426+273$ & $03-30-2007$ & 0.65 & 0.29 & 0 & 0.74 & 0.79 & 1.1 & $3.4 \times\left(1, \ldots, 2^{7}\right)$ \\
\hline
\end{tabular}


TABLE $2-$ Continued

\begin{tabular}{|c|c|c|c|c|c|c|c|c|}
\hline \multirow[b]{2}{*}{ Source } & \multirow[b]{2}{*}{$\begin{array}{l}\text { Observation } \\
\text { Session }\end{array}$} & \multicolumn{3}{|c|}{ Beam $^{\mathrm{a}}$} & \multicolumn{3}{|c|}{$\begin{array}{l}\text { Flux Density } \\
\end{array}$} & \multirow[b]{2}{*}{$\begin{array}{l}\text { Contour Levels } \\
\left(\mathrm{mJy} \mathrm{bm}^{-1}\right)\end{array}$} \\
\hline & & $\begin{array}{c}a \\
\text { (mas) }\end{array}$ & $\begin{array}{c}b \\
\text { (mas) }\end{array}$ & $\begin{array}{l}\phi \\
\left(^{\circ}\right)\end{array}$ & $\begin{array}{c}S_{\text {peak }} \\
\left(\mathrm{Jy} \mathrm{bm}^{-1}\right)\end{array}$ & $\begin{array}{c}S_{\text {total }}^{\text {b }} \\
\text { (Jy) }\end{array}$ & $\begin{array}{c}S_{\mathrm{rms}}{ }^{\mathrm{c}} \\
\left(\mathrm{mJy} \mathrm{bm}^{-1}\right)\end{array}$ & \\
\hline $0426-380$ & $05-22-2003$ & 0.73 & 0.26 & 1 & 0.61 & 0.72 & 4.3 & $12.4 \times\left(1, \ldots, 2^{5}\right)$ \\
\hline $0429+174$ & $03-30-2007$ & 0.69 & 0.32 & -6 & 0.26 & 0.30 & 1.2 & $3.5 \times\left(1, \ldots, 2^{6}\right)$ \\
\hline $0430+052$ & 03-30-2007 & 0.69 & 0.27 & 0 & 1.17 & 2.50 & 5.5 & $16.5 \times\left(1, \ldots, 2^{6}\right)$ \\
\hline $0446+112$ & $12-14-2004$ & 0.54 & 0.29 & -17 & 1.12 & 1.39 & 2.9 & $8.8 \times\left(1, \ldots, 2^{6}\right)$ \\
\hline $0450+013$ & $05-22-2003$ & 0.80 & 0.31 & -9 & 0.25 & 0.27 & 2.0 & $5.3 \times\left(1, \ldots, 2^{5}\right)$ \\
\hline $0454-234$ & 03-30-2007 & 0.73 & 0.29 & -4 & 2.41 & 2.91 & 6.9 & $20.6 \times\left(1, \ldots, 2^{6}\right)$ \\
\hline $0458-020$ & 03-30-2007 & 0.75 & 0.28 & -3 & 0.66 & 0.76 & 2.0 & $6.0 \times\left(1, \ldots, 2^{6}\right)$ \\
\hline $0458+138$ & 03-30-2007 & 0.95 & 0.43 & 12 & 0.22 & 0.26 & 2.3 & $6.6 \times\left(1, \ldots, 2^{5}\right)$ \\
\hline $0459+135$ & 03-30-2007 & 0.73 & 0.29 & 2 & 0.43 & 0.60 & 1.5 & $4.6 \times\left(1, \ldots, 2^{6}\right)$ \\
\hline $0502+049$ & 03-30-2007 & 0.72 & 0.31 & -4 & 0.62 & 0.73 & 1.6 & $4.8 \times\left(1, \ldots, 2^{7}\right)$ \\
\hline $0506+056$ & 03-30-2007 & 0.78 & 0.31 & -8 & 0.52 & 0.65 & 1.7 & $5.1 \times\left(1, \ldots, 2^{6}\right)$ \\
\hline $0507+179$ & 03-30-2007 & 0.68 & 0.30 & -4 & 0.76 & 0.86 & 1.3 & $4.0 \times\left(1, \ldots, 2^{7}\right)$ \\
\hline $0511-220$ & 03-30-2007 & 0.77 & 0.29 & -8 & 0.58 & 0.74 & 2.6 & $7.9 \times\left(1, \ldots, 2^{6}\right)$ \\
\hline $0524+034$ & 03-30-2007 & 0.73 & 0.30 & -4 & 0.43 & 0.45 & 1.5 & $4.6 \times\left(1, \ldots, 2^{6}\right)$ \\
\hline $0528+134$ & $12-14-2004$ & 0.60 & 0.29 & -18 & 0.96 & 1.91 & 3.3 & $9.8 \times\left(1, \ldots, 2^{6}\right)$ \\
\hline $0528-250$ & $05-22-2003$ & 2.18 & 0.31 & -22 & 0.26 & 0.32 & 3.3 & $10.0 \times\left(1, \ldots, 2^{4}\right)$ \\
\hline $0534-340$ & $05-22-2003$ & 1.19 & 0.29 & -14 & 0.31 & 0.37 & 3.4 & $10.2 \times\left(1, \ldots, 2^{4}\right)$ \\
\hline $0537-441$ & 05-22-2003 & 0.71 & 0.25 & -1 & 2.15 & 2.73 & 7.3 & $21.8 \times\left(1, \ldots, 2^{6}\right)$ \\
\hline $0544+273$ & 03-30-2007 & 0.63 & 0.33 & -1 & 0.30 & 0.32 & 1.0 & $3.1 \times\left(1, \ldots, 2^{6}\right)$ \\
\hline $0547+234$ & 03-30-2007 & 0.64 & 0.32 & -7 & 0.22 & 0.25 & 1.1 & $3.2 \times\left(1, \ldots, 2^{6}\right)$ \\
\hline $0549+192$ & 03-30-2007 & 0.79 & 0.34 & -13 & 0.18 & 0.21 & 1.3 & $3.8 \times\left(1, \ldots, 2^{5}\right)$ \\
\hline $0552+398$ & $12-14-2004$ & 0.74 & 0.57 & -52 & 1.49 & 1.99 & 4.0 & $12.0 \times\left(1, \ldots, 2^{6}\right)$ \\
\hline $0554+242$ & $03-30-2007$ & 0.89 & 0.55 & -1 & 0.26 & 0.40 & 1.3 & $3.9 \times\left(1, \ldots, 2^{6}\right)$ \\
\hline $0556+238$ & 08-26-2005 & 1.07 & 0.33 & -17 & 0.32 & 0.38 & 1.6 & $4.9 \times\left(1, \ldots, 2^{6}\right)$ \\
\hline $0601+245$ & $08-26-2005$ & 1.74 & 0.45 & -5 & 0.40 & 0.50 & 8.3 & $24.8 \times\left(1, \ldots, 2^{4}\right)$ \\
\hline $0607-157$ & 03-30-2007 & 1.04 & 0.35 & -5 & 1.29 & 1.76 & 7.4 & $22.1 \times\left(1, \ldots, 2^{5}\right)$ \\
\hline $0611+131$ & 03-30-2007 & 0.70 & 0.30 & -6 & 0.31 & 0.37 & 1.1 & $3.2 \times\left(1, \ldots, 2^{6}\right)$ \\
\hline $0621+446$ & $05-22-2003$ & 0.85 & 0.28 & -27 & 0.20 & 0.22 & 2.4 & $7.2 \times\left(1, \ldots, 2^{4}\right)$ \\
\hline $0639-032$ & 03-30-2007 & 0.83 & 0.36 & -1 & 0.30 & 0.36 & 2.1 & $6.0 \times\left(1, \ldots, 2^{5}\right)$ \\
\hline $0639+352$ & 05-22-2003 & 1.66 & 0.28 & -16 & 0.22 & 0.25 & 4.7 & $14.1 \times\left(1, \ldots, 2^{3}\right)$ \\
\hline $0642+449$ & $12-14-2004$ & 0.68 & 0.38 & 36 & 1.57 & 2.44 & 3.9 & $11.8 \times\left(1, \ldots, 2^{7}\right)$ \\
\hline $0646-306$ & 09-13-2003 & 1.43 & 0.30 & -21 & 0.38 & 0.47 & 2.8 & $8.4 \times\left(1, \ldots, 2^{5}\right)$ \\
\hline $0648-165$ & 03-30-2007 & 0.76 & 0.30 & 4 & 1.45 & 2.04 & 2.4 & $7.2 \times\left(1, \ldots, 2^{7}\right)$ \\
\hline $0654+244$ & 08-26-2005 & 0.93 & 0.27 & 0 & 0.26 & 0.31 & 3.9 & $11.7 \times\left(1, \ldots, 2^{4}\right)$ \\
\hline $0707+476$ & $03-30-2007$ & 0.74 & 0.31 & -25 & 0.43 & 0.52 & 1.7 & $5.0 \times\left(1, \ldots, 2^{6}\right)$ \\
\hline $0716+477$ & 03-30-2007 & 0.63 & 0.42 & -4 & 0.31 & 0.41 & 1.2 & $3.6 \times\left(1, \ldots, 2^{6}\right)$ \\
\hline $0721-071$ & 03-30-2007 & 0.73 & 0.28 & 0 & 0.48 & 0.61 & 2.5 & $7.4 \times\left(1, \ldots, 2^{6}\right)$ \\
\hline $0722+145$ & 03-30-2007 & 0.73 & 0.32 & -11 & 0.51 & 0.58 & 2.2 & $6.5 \times\left(1, \ldots, 2^{6}\right)$ \\
\hline $0725+219$ & 03-30-2007 & 0.81 & 0.31 & -11 & 0.20 & 0.25 & 1.5 & $4.5 \times\left(1, \ldots, 2^{5}\right)$ \\
\hline $0727-115$ & $12-14-2004$ & 0.73 & 0.28 & 1 & 2.23 & 3.32 & 12.0 & $36.0 \times\left(1, \ldots, 2^{5}\right)$ \\
\hline $0735+178$ & 03-30-2007 & 0.67 & 0.31 & -10 & 0.36 & 0.61 & 1.6 & $4.9 \times\left(1, \ldots, 2^{6}\right)$ \\
\hline $0736+017$ & 03-30-2007 & 0.79 & 0.32 & -7 & 1.78 & 2.08 & 3.1 & $9.2 \times\left(1, \ldots, 2^{7}\right)$ \\
\hline $0743-006$ & $12-14-2004$ & 0.89 & 0.35 & 19 & 0.68 & 0.83 & 7.1 & $21.4 \times\left(1, \ldots, 2^{4}\right)$ \\
\hline $0745+241$ & 03-30-2007 & 0.70 & 0.28 & -2 & 0.88 & 1.19 & 1.2 & $3.6 \times\left(1, \ldots, 2^{7}\right)$ \\
\hline $0746+483$ & 03-30-2007 & 0.54 & 0.33 & -16 & 0.39 & 0.59 & 1.3 & $3.8 \times\left(1, \ldots, 2^{6}\right)$ \\
\hline $0748+126$ & 03-30-2007 & 0.72 & 0.28 & -2 & 2.40 & 3.27 & 3.3 & $10.0 \times\left(1, \ldots, 2^{7}\right)$ \\
\hline $0749+540$ & 03-30-2007 & 0.56 & 0.30 & -2 & 0.68 & 0.82 & 1.0 & $3.1 \times\left(1, \ldots, 2^{7}\right)$ \\
\hline $0754+100$ & 03-30-2007 & 0.72 & 0.28 & -1 & 0.91 & 1.24 & 2.2 & $6.6 \times\left(1, \ldots, 2^{7}\right)$ \\
\hline $0759+183$ & $03-30-2007$ & 0.97 & 0.34 & -19 & 0.24 & 0.32 & 1.4 & $4.1 \times\left(1, \ldots, 2^{5}\right)$ \\
\hline $0804+499$ & 03-30-2007 & 0.54 & 0.32 & -14 & 0.57 & 0.64 & 1.0 & $3.0 \times\left(1, \ldots, 2^{7}\right)$ \\
\hline $0805-077$ & 03-30-2007 & 0.84 & 0.31 & -5 & 0.95 & 1.09 & 2.3 & $6.9 \times\left(1, \ldots, 2^{7}\right)$ \\
\hline $0805+410$ & $12-14-2004$ & 0.54 & 0.41 & 18 & 0.59 & 0.66 & 1.4 & $4.1 \times\left(1, \ldots, 2^{7}\right)$ \\
\hline $0808+019$ & 03-30-2007 & 0.80 & 0.32 & -7 & 0.51 & 0.55 & 1.7 & $5.1 \times\left(1, \ldots, 2^{6}\right)$ \\
\hline $0812+367$ & 03-30-2007 & 0.58 & 0.31 & -7 & 0.33 & 0.48 & 1.2 & $3.7 \times\left(1, \ldots, 2^{6}\right)$ \\
\hline $0814+425$ & $12-14-2004$ & 0.54 & 0.34 & -20 & 0.38 & 0.48 & 2.0 & $6.0 \times\left(1, \ldots, 2^{6}\right)$ \\
\hline $0820+560$ & $12-14-2004$ & 0.49 & 0.40 & 20 & 0.31 & 0.46 & 1.5 & $4.4 \times\left(1, \ldots, 2^{6}\right)$ \\
\hline $0821+248$ & $03-30-2007$ & 0.71 & 0.29 & -1 & 0.23 & 0.25 & 1.4 & $4.2 \times\left(1, \ldots, 2^{5}\right)$ \\
\hline $0821+394$ & $03-30-2007$ & 0.64 & 0.29 & 4 & 0.82 & 1.01 & 1.4 & $4.1 \times\left(1, \ldots, 2^{7}\right)$ \\
\hline $0823+033$ & $12-14-2004$ & 0.82 & 0.35 & 17 & 1.24 & 1.36 & 2.4 & $7.3 \times\left(1, \ldots, 2^{7}\right)$ \\
\hline $0827+243$ & 03-30-2007 & 0.70 & 0.29 & 3 & 0.53 & 0.62 & 1.3 & $3.8 \times\left(1, \ldots, 2^{7}\right)$ \\
\hline $0834-201$ & 03-30-2007 & 0.77 & 0.29 & -4 & 0.97 & 1.52 & 3.3 & $9.9 \times\left(1, \ldots, 2^{6}\right)$ \\
\hline $0834+250$ & 03-30-2007 & 0.69 & 0.29 & 3 & 0.31 & 0.45 & 1.5 & $4.2 \times\left(1, \ldots, 2^{6}\right)$ \\
\hline $0838+133$ & 03-30-2007 & 0.70 & 0.29 & -2 & 0.56 & 0.97 & 1.1 & $3.2 \times\left(1, \ldots, 2^{7}\right)$ \\
\hline $0839+187$ & 03-30-2007 & 1.59 & 0.58 & -13 & 0.18 & 0.22 & 4.2 & $12.5 \times\left(1, \ldots, 2^{3}\right)$ \\
\hline $0850+581$ & 03-30-2007 & 0.73 & 0.36 & -20 & 0.19 & 0.29 & 1.1 & $3.4 \times\left(1, \ldots, 2^{5}\right)$ \\
\hline $0851+202$ & $12-14-2004$ & 0.71 & 0.36 & 22 & 2.07 & 2.44 & 11.0 & $30.7 \times\left(1, \ldots, 2^{6}\right)$ \\
\hline $0906+163$ & 03-30-2007 & 0.99 & 0.36 & -28 & 0.21 & 0.23 & 2.2 & $6.7 \times\left(1, \ldots, 2^{4}\right)$ \\
\hline $0912+029$ & 03-30-2007 & 0.77 & 0.30 & -6 & 0.82 & 1.06 & 2.3 & $6.9 \times\left(1, \ldots, 2^{6}\right)$ \\
\hline
\end{tabular}


TABLE 2 - Continued

\begin{tabular}{|c|c|c|c|c|c|c|c|c|}
\hline \multirow[b]{2}{*}{ Source } & \multirow[b]{2}{*}{$\begin{array}{c}\text { Observation } \\
\text { Session }\end{array}$} & \multicolumn{3}{|c|}{ Beam $^{a}$} & \multicolumn{3}{|c|}{ Flux Density } & \multirow[b]{2}{*}{$\begin{array}{l}\text { Contour Levels } \\
\left(\mathrm{mJy} \mathrm{bm}^{-1}\right)\end{array}$} \\
\hline & & $\begin{array}{c}a \\
(\mathrm{mas})\end{array}$ & $\begin{array}{c}b \\
(\mathrm{mas})\end{array}$ & $\begin{array}{c}\phi \\
\left({ }^{\circ}\right)\end{array}$ & $\begin{array}{c}S_{\text {peak }} \\
\left(\mathrm{Jy} \mathrm{bm}^{-1}\right)\end{array}$ & $\begin{array}{c}S_{\text {total }^{\mathrm{b}}} \\
(\mathrm{Jy})\end{array}$ & $\begin{array}{c}S_{\mathrm{rms}}{ }^{\mathrm{c}} \\
\left(\mathrm{mJy} \mathrm{bm}^{-1}\right)\end{array}$ & \\
\hline $0917+624$ & $12-14-2004$ & 0.44 & 0.38 & 66 & 0.49 & 0.58 & 1.3 & $4.0 \times\left(1, \ldots, 2^{6}\right)$ \\
\hline $0923+392$ & $12-14-2004$ & 0.62 & 0.29 & -5 & 1.71 & 6.25 & 4.5 & $13.4 \times\left(1, \ldots, 2^{6}\right)$ \\
\hline $0945+408$ & $03-30-2007$ & 0.72 & 0.30 & -14 & 1.02 & 1.32 & 1.7 & $5.2 \times\left(1, \ldots, 2^{7}\right)$ \\
\hline $0953+254$ & $03-30-2007$ & 0.69 & 0.28 & -13 & 0.72 & 1.00 & 1.8 & $5.5 \times\left(1, \ldots, 2^{7}\right)$ \\
\hline $0955+326$ & $03-30-2007$ & 0.66 & 0.31 & -7 & 0.50 & 0.70 & 1.5 & $4.5 \times\left(1, \ldots, 2^{6}\right)$ \\
\hline $0955+476$ & $05-22-2003$ & 0.84 & 0.30 & -32 & 0.81 & 1.10 & 1.8 & $5.3 \times\left(1, \ldots, 2^{7}\right)$ \\
\hline $1005+066$ & $03-30-2007$ & 0.76 & 0.30 & -8 & 0.61 & 0.66 & 2.3 & $6.8 \times\left(1, \ldots, 2^{6}\right)$ \\
\hline $1012+232$ & $03-30-2007$ & 0.86 & 0.28 & -27 & 0.46 & 0.72 & 2.5 & $7.6 \times\left(1, \ldots, 2^{5}\right)$ \\
\hline $1016-311$ & $03-30-2007$ & 0.92 & 0.32 & -9 & 0.41 & 0.51 & 3.5 & $10.6 \times\left(1, \ldots, 2^{5}\right)$ \\
\hline $1020+400$ & $03-30-2007$ & 0.71 & 0.29 & -8 & 0.39 & 0.51 & 1.2 & $3.5 \times\left(1, \ldots, 2^{6}\right)$ \\
\hline $1022+194$ & 03-30-2007 & 0.85 & 0.34 & -22 & 0.25 & 0.33 & 1.5 & $4.6 \times\left(1, \ldots, 2^{5}\right)$ \\
\hline $1022+237$ & $05-22-2003$ & 1.71 & 0.96 & -48 & 0.29 & 0.32 & 2.3 & $6.4 \times\left(1, \ldots, 2^{5}\right)$ \\
\hline $1032-199$ & $03-30-2007$ & 0.73 & 0.27 & 3 & 0.58 & 1.14 & 4.5 & $13.4 \times\left(1, \ldots, 2^{5}\right)$ \\
\hline $1034-293$ & $03-30-2007$ & 0.77 & 0.28 & -2 & 1.66 & 2.10 & 5.1 & $15.4 \times\left(1, \ldots, 2^{6}\right)$ \\
\hline $1039+811$ & $03-30-2007$ & 0.42 & 0.38 & 35 & 0.48 & 0.81 & 1.7 & $5.2 \times\left(1, \ldots, 2^{6}\right)$ \\
\hline $1045-188$ & $03-30-2007$ & 0.75 & 0.28 & -4 & 0.84 & 1.10 & 2.4 & $7.2 \times\left(1, \ldots, 2^{6}\right)$ \\
\hline $1048-313$ & $05-22-2003$ & 3.22 & 0.31 & -18 & 0.36 & 0.47 & 2.7 & $8.2 \times\left(1, \ldots, 2^{5}\right)$ \\
\hline $1049+215$ & $03-30-2007$ & 0.76 & 0.29 & -9 & 0.37 & 0.49 & 3.2 & $9.6 \times\left(1, \ldots, 2^{5}\right)$ \\
\hline $1053+815$ & $03-30-2007$ & 0.41 & 0.37 & -28 & 0.79 & 0.86 & 0.8 & $2.3 \times\left(1, \ldots, 2^{8}\right)$ \\
\hline $1055+018$ & $03-30-2007$ & 0.72 & 0.30 & -3 & 4.54 & 6.23 & 6.6 & $19.9 \times\left(1, \ldots, 2^{7}\right)$ \\
\hline $1101+384$ & $03-30-2007$ & 0.68 & 0.30 & -4 & 0.34 & 0.39 & 1.4 & $4.3 \times\left(1, \ldots, 2^{6}\right)$ \\
\hline $1113+087$ & $03-30-2007$ & 1.07 & 0.42 & -21 & 0.25 & 0.34 & 2.5 & $7.5 \times\left(1, \ldots, 2^{5}\right)$ \\
\hline $1124-186$ & 03-30-2007 & 0.76 & 0.28 & -1 & 1.20 & 1.35 & 2.7 & $8.0 \times\left(1, \ldots, 2^{7}\right)$ \\
\hline $1128+385$ & $03-30-2007$ & 0.75 & 0.28 & -2 & 0.86 & 1.05 & 1.5 & $4.4 \times\left(1, \ldots, 2^{7}\right)$ \\
\hline $1142+052$ & $03-30-2007$ & 1.00 & 0.36 & -18 & 0.20 & 0.23 & 2.0 & $6.1 \times\left(1, \ldots, 2^{5}\right)$ \\
\hline $1144-379$ & $05-22-2003$ & 0.77 & 0.31 & -3 & 0.76 & 1.05 & 4.5 & $13.5 \times\left(1, \ldots, 2^{5}\right)$ \\
\hline $1144+402$ & $12-14-2004$ & 0.66 & 0.35 & 25 & 0.95 & 1.04 & 1.9 & $5.6 \times\left(1, \ldots, 2^{7}\right)$ \\
\hline $1147+245$ & $03-30-2007$ & 0.71 & 0.30 & -10 & 0.38 & 0.63 & 2.5 & $7.0 \times\left(1, \ldots, 2^{5}\right)$ \\
\hline $1150+497$ & 03-30-2007 & 0.56 & 0.33 & -2 & 0.89 & 1.26 & 1.8 & $5.1 \times\left(1, \ldots, 2^{7}\right)$ \\
\hline $1150+812$ & $03-30-2007$ & 0.43 & 0.36 & 2 & 0.62 & 1.21 & 2.2 & $6.5 \times\left(1, \ldots, 2^{6}\right)$ \\
\hline $1156+295$ & $03-30-2007$ & 0.67 & 0.33 & -20 & 0.97 & 1.03 & 1.7 & $5.2 \times\left(1, \ldots, 2^{7}\right)$ \\
\hline $1207-319$ & $05-22-2003$ & 2.67 & 0.30 & -16 & 0.34 & 0.40 & 3.4 & $10.3 \times\left(1, \ldots, 2^{5}\right)$ \\
\hline $1213-172$ & $03-30-2007$ & 0.74 & 0.29 & -1 & 1.03 & 1.49 & 2.3 & $7.0 \times\left(1, \ldots, 2^{7}\right)$ \\
\hline $1236+077$ & $03-30-2007$ & 0.73 & 0.29 & -5 & 0.84 & 1.05 & 2.1 & $6.2 \times\left(1, \ldots, 2^{7}\right)$ \\
\hline $1243-072$ & $03-30-2007$ & 0.74 & 0.30 & -2 & 0.89 & 0.94 & 1.5 & $4.5 \times\left(1, \ldots, 2^{7}\right)$ \\
\hline $1256-220$ & $03-30-2007$ & 0.81 & 0.30 & -10 & 0.37 & 0.52 & 2.1 & $6.4 \times\left(1, \ldots, 2^{5}\right)$ \\
\hline $1302-102$ & $12-26-2002$ & 0.81 & 0.27 & -7 & 0.38 & 0.52 & 1.8 & $5.1 \times\left(1, \ldots, 2^{6}\right)$ \\
\hline $1308+326$ & $03-30-2007$ & 0.66 & 0.30 & 9 & 0.79 & 0.98 & 2.0 & $6.1 \times\left(1, \ldots, 2^{7}\right)$ \\
\hline $1308+554$ & $03-30-2007$ & 0.69 & 0.41 & 7 & 0.18 & 0.20 & 1.1 & $3.3 \times\left(1, \ldots, 2^{5}\right)$ \\
\hline $1313-333$ & $12-14-2004$ & 0.36 & 0.19 & -48 & 0.79 & 1.10 & 4.5 & $13.5 \times\left(1, \ldots, 2^{5}\right)$ \\
\hline $1324+224$ & $12-14-2004$ & 0.70 & 0.33 & 15 & 0.92 & 1.10 & 1.4 & $4.1 \times\left(1, \ldots, 2^{7}\right)$ \\
\hline $1334-127$ & $12-14-2004$ & 0.74 & 0.31 & 9 & 4.87 & 6.26 & 9.6 & $28.8 \times\left(1, \ldots, 2^{7}\right)$ \\
\hline $1354-152$ & $03-30-2007$ & 0.74 & 0.28 & -3 & 0.42 & 0.49 & 1.6 & $4.9 \times\left(1, \ldots, 2^{6}\right)$ \\
\hline $1357+769$ & $05-22-2003$ & 0.73 & 0.28 & 12 & 0.58 & 0.66 & 1.9 & $5.8 \times\left(1, \ldots, 2^{6}\right)$ \\
\hline $1406-076$ & $05-22-2003$ & 0.71 & 0.29 & 2 & 0.76 & 1.04 & 2.4 & $7.2 \times\left(1, \ldots, 2^{6}\right)$ \\
\hline $1406-267$ & $05-22-2003$ & 3.95 & 0.50 & 28 & 0.59 & 0.64 & 3.7 & $11.0 \times\left(1, \ldots, 2^{5}\right)$ \\
\hline $1418+546$ & 03-30-2007 & 0.64 & 0.30 & -4 & 0.63 & 0.98 & 1.6 & $4.9 \times\left(1, \ldots, 2^{7}\right)$ \\
\hline $1435-218$ & $03-30-2007$ & 0.73 & 0.29 & -6 & 0.75 & 0.88 & 2.2 & $6.5 \times\left(1, \ldots, 2^{6}\right)$ \\
\hline $1437-153$ & 03-30-2007 & 0.72 & 0.29 & -2 & 0.42 & 0.46 & 2.0 & $6.1 \times\left(1, \ldots, 2^{6}\right)$ \\
\hline $1451-375$ & $05-22-2003$ & 0.93 & 0.29 & $-\overline{9}$ & 0.51 & 0.61 & 5.3 & $14.3 \times\left(1, \ldots, 2^{5}\right)$ \\
\hline $1459+480$ & $03-30-2007$ & 0.62 & 0.34 & 0 & 0.26 & 0.34 & 1.3 & $3.9 \times\left(1, \ldots, 2^{6}\right)$ \\
\hline $1502+036$ & $05-22-2003$ & 0.76 & 0.28 & -5 & 0.38 & 0.46 & 2.2 & $6.0 \times\left(1, \ldots, 2^{6}\right)$ \\
\hline $1502+106$ & $03-30-2007$ & 0.72 & 0.30 & -8 & 1.02 & 1.34 & 2.8 & $8.3 \times\left(1, \ldots, 2^{6}\right)$ \\
\hline $1504+377$ & $05-22-2003$ & 0.84 & 0.37 & -27 & 0.29 & 0.38 & 2.3 & $7.0 \times\left(1, \ldots, 2^{5}\right)$ \\
\hline $1505+428$ & 03-30-2007 & 0.58 & 0.33 & 0 & 0.35 & 0.49 & 1.1 & $3.3 \times\left(1, \ldots, 2^{6}\right)$ \\
\hline $1511-100$ & $12-14-2004$ & 0.49 & 0.28 & -21 & 0.94 & 1.24 & 2.3 & $7.0 \times\left(1, \ldots, 2^{7}\right)$ \\
\hline $1514+004$ & $03-30-2007$ & 0.84 & 0.31 & -8 & 0.62 & 0.86 & 2.1 & $6.4 \times\left(1, \ldots, 2^{6}\right)$ \\
\hline $1514+197$ & $03-30-2007$ & 0.71 & 0.29 & -2 & 0.62 & 0.71 & 1.7 & $5.1 \times\left(1, \ldots, 2^{6}\right)$ \\
\hline $1514-241$ & 03-30-2007 & 0.75 & 0.28 & -1 & 1.61 & 2.74 & 5.1 & $15.2 \times\left(1, \ldots, 2^{6}\right)$ \\
\hline $1519-273$ & 03-30-2007 & 0.73 & 0.28 & -2 & 1.02 & 1.24 & 2.6 & $7.7 \times\left(1, \ldots, 2^{7}\right)$ \\
\hline $1520+437$ & $05-22-2003$ & 2.14 & 0.39 & 6 & 0.24 & 0.25 & 2.7 & $8.1 \times\left(1, \ldots, 2^{4}\right)$ \\
\hline $1546+027$ & $12-14-2004$ & 0.73 & 0.31 & -4 & 3.10 & 3.49 & 1.8 & $5.5 \times\left(1, \ldots, 2^{9}\right)$ \\
\hline $1548+056$ & $03-30-2007$ & 0.77 & 0.34 & -5 & 1.01 & 2.36 & 6.5 & $19.6 \times\left(1, \ldots, 2^{5}\right)$ \\
\hline $1606+106$ & $12-14-2004$ & 0.52 & 0.26 & -26 & 1.05 & 1.28 & 2.6 & $7.7 \times\left(1, \ldots, 2^{7}\right)$ \\
\hline $1611+343$ & $03-30-2007$ & 0.70 & 0.28 & 9 & 2.02 & 2.91 & 4.4 & $13.3 \times\left(1, \ldots, 2^{7}\right)$ \\
\hline $1617+229$ & $03-30-2007$ & 0.67 & 0.32 & -4 & 0.32 & 0.55 & 1.2 & $3.7 \times\left(1, \ldots, 2^{6}\right)$ \\
\hline $1622-253$ & $03-30-2007$ & 0.72 & 0.27 & 1 & 1.32 & 1.65 & 5.0 & $15.1 \times\left(1, \ldots, 2^{6}\right)$ \\
\hline $1622-297$ & $03-30-2007$ & 0.75 & 0.27 & -3 & 1.28 & 1.76 & 6.8 & $20.5 \times\left(1, \ldots, 2^{5}\right)$ \\
\hline
\end{tabular}


TABLE 2 - Continued

\begin{tabular}{|c|c|c|c|c|c|c|c|c|}
\hline \multirow[b]{2}{*}{ Source } & \multirow[b]{2}{*}{$\begin{array}{l}\text { Observation } \\
\text { Session }\end{array}$} & \multicolumn{3}{|c|}{ Beam $^{\mathrm{a}}$} & \multicolumn{3}{|c|}{$\begin{array}{l}\text { Flux Density } \\
\end{array}$} & \multirow[b]{2}{*}{$\begin{array}{l}\text { Contour Levels } \\
\left(\mathrm{mJy} \mathrm{bm}^{-1}\right)\end{array}$} \\
\hline & & $\begin{array}{c}a \\
\text { (mas) }\end{array}$ & $\begin{array}{c}b \\
\text { (mas) }\end{array}$ & $\begin{array}{l}\phi \\
\left({ }^{\circ}\right)\end{array}$ & $\begin{array}{c}S_{\text {peak }} \\
\left(\mathrm{Jy} \mathrm{bm}^{-1}\right)\end{array}$ & $\begin{array}{c}S_{\text {total }^{b}} \\
(\mathrm{Jy})\end{array}$ & $\begin{array}{c}S_{\mathrm{rms}}{ }^{\mathrm{c}} \\
\left(\mathrm{mJy} \mathrm{bm}^{-1}\right)\end{array}$ & \\
\hline $1637+574$ & $12-14-2004$ & 0.73 & 0.35 & 32 & 0.72 & 0.96 & 1.2 & $3.6 \times\left(1, \ldots, 2^{7}\right)$ \\
\hline $1638+398$ & $12-14-2004$ & 0.64 & 0.43 & 15 & 0.69 & 0.91 & 1.4 & $3.9 \times\left(1, \ldots, 2^{7}\right)$ \\
\hline $1652+398$ & $03-30-2007$ & 0.57 & 0.33 & 6 & 0.44 & 0.71 & 1.3 & $3.8 \times\left(1, \ldots, 2^{6}\right)$ \\
\hline $1656-075$ & $03-30-2007$ & 1.06 & 0.36 & -11 & 0.26 & 0.37 & 1.8 & $5.5 \times\left(1, \ldots, 2^{5}\right)$ \\
\hline $1657-261$ & 03-30-2007 & 0.76 & 0.28 & -6 & 0.42 & 0.50 & 2.0 & $6.0 \times\left(1, \ldots, 2^{6}\right)$ \\
\hline $1705+018$ & 03-30-2007 & 0.75 & 0.31 & -5 & 0.58 & 0.66 & 1.6 & $4.9 \times\left(1, \ldots, 2^{6}\right)$ \\
\hline $1710-269$ & 03-30-2007 & 1.51 & 0.49 & -16 & 0.41 & 0.81 & 7.6 & $20.4 \times\left(1, \ldots, 2^{4}\right)$ \\
\hline $1717+178$ & 03-30-2007 & 0.67 & 0.26 & 3 & 0.49 & 0.70 & 2.1 & $6.2 \times\left(1, \ldots, 2^{6}\right)$ \\
\hline $1726+455$ & 03-30-2007 & 0.50 & 0.34 & 11 & 0.37 & 0.38 & 1.3 & $4.0 \times\left(1, \ldots, 2^{6}\right)$ \\
\hline $1730-130$ & 03-30-2007 & 0.73 & 0.29 & -1 & 1.40 & 2.31 & 3.0 & $9.0 \times\left(1, \ldots, 2^{7}\right)$ \\
\hline $1732+389$ & $12-14-2004$ & 0.74 & 0.30 & 12 & 0.87 & 1.11 & 1.1 & $3.2 \times\left(1, \ldots, 2^{8}\right)$ \\
\hline $1738+476$ & 03-30-2007 & 0.54 & 0.34 & 6 & 0.40 & 0.58 & 1.6 & $4.9 \times\left(1, \ldots, 2^{6}\right)$ \\
\hline $1741-038$ & $12-14-2004$ & 0.76 & 0.33 & 2 & 3.70 & 5.60 & 3.1 & $9.2 \times\left(1, \ldots, 2^{8}\right)$ \\
\hline $1741-312$ & 03-30-2007 & 4.51 & 1.13 & 17 & 0.56 & 0.66 & 6.9 & $20.8 \times\left(1, \ldots, 2^{4}\right)$ \\
\hline $1742-078$ & 03-30-2007 & 0.73 & 0.28 & -3 & 0.50 & 0.66 & 1.8 & $5.4 \times\left(1, \ldots, 2^{6}\right)$ \\
\hline $1749+096$ & $12-14-2004$ & 0.58 & 0.26 & -31 & 2.77 & 3.37 & 10.0 & $29.9 \times\left(1, \ldots, 2^{6}\right)$ \\
\hline $1749+701$ & $03-30-2007$ & 0.80 & 0.47 & 89 & 0.24 & 0.35 & 1.8 & $5.4 \times\left(1, \ldots, 2^{5}\right)$ \\
\hline $1751+288$ & 03-30-2007 & 0.70 & 0.28 & 13 & 1.68 & 1.97 & 3.4 & $10.2 \times\left(1, \ldots, 2^{7}\right)$ \\
\hline $1754+155$ & 03-30-2007 & 0.72 & 0.29 & 6 & 0.39 & 0.44 & 1.1 & $3.3 \times\left(1, \ldots, 2^{6}\right)$ \\
\hline $1800+440$ & 03-30-2007 & 0.54 & 0.33 & 6 & 1.52 & 1.72 & 2.0 & $6.1 \times\left(1, \ldots, 2^{7}\right)$ \\
\hline $1803+784$ & $12-14-2004$ & 0.53 & 0.41 & 82 & 0.89 & 1.22 & 1.2 & $3.6 \times\left(1, \ldots, 2^{7}\right)$ \\
\hline $1817-254$ & 03-30-2007 & 0.76 & 0.28 & -1 & 0.49 & 0.67 & 2.5 & $7.6 \times\left(1, \ldots, 2^{6}\right)$ \\
\hline $1817+387$ & 03-30-2007 & 0.65 & 0.35 & 11 & 0.20 & 0.22 & 1.2 & $3.7 \times\left(1, \ldots, 2^{5}\right)$ \\
\hline $1829-106$ & 03-30-2007 & 1.67 & 0.97 & 17 & 0.37 & 0.62 & 6.8 & $20.3 \times\left(1, \ldots, 2^{4}\right)$ \\
\hline $1829-207$ & 03-30-2007 & 2.07 & 0.58 & -1 & 0.33 & 0.41 & 4.5 & $12.2 \times\left(1, \ldots, 2^{4}\right)$ \\
\hline $1830-211$ & 03-30-2007 & 2.96 & 0.97 & -3 & 2.82 & 3.11 & 33.6 & $90.8 \times\left(1, \ldots, 2^{4}\right)$ \\
\hline $1849+670$ & $12-14-2004$ & 0.54 & 0.35 & 28 & 0.41 & 0.45 & 1.1 & $3.4 \times\left(1, \ldots, 2^{6}\right)$ \\
\hline $1901+319$ & $12-14-2004$ & 0.69 & 0.33 & 5 & 0.31 & 0.65 & 1.5 & $4.6 \times\left(1, \ldots, 2^{6}\right)$ \\
\hline $1921-293$ & $03-30-2007$ & 0.74 & 0.29 & -5 & 7.10 & 9.43 & 8.0 & $24.1 \times\left(1, \ldots, 2^{8}\right)$ \\
\hline $1928+154$ & 03-30-2007 & 0.70 & 0.29 & 1 & 0.32 & 0.36 & 1.6 & $4.7 \times\left(1, \ldots, 2^{6}\right)$ \\
\hline $1936-155$ & $02-15-2004$ & 0.73 & 0.28 & -2 & 0.51 & 0.58 & 1.4 & $4.2 \times\left(1, \ldots, 2^{6}\right)$ \\
\hline $1946-200$ & 05-22-2003 & 2.44 & 0.52 & 26 & 0.38 & 0.36 & 4.5 & $13.5 \times\left(1, \ldots, 2^{4}\right)$ \\
\hline $1954-388$ & $05-22-2003$ & 0.68 & 0.25 & -1 & 1.70 & 2.21 & 5.2 & $16.5 \times\left(1, \ldots, 2^{6}\right)$ \\
\hline $1958-179$ & $05-22-2003$ & 0.71 & 0.27 & -2 & 1.28 & 1.54 & 3.1 & $8.5 \times\left(1, \ldots, 2^{7}\right)$ \\
\hline $2000+472$ & $03-30-2007$ & 0.53 & 0.33 & 14 & 0.36 & 0.52 & 1.3 & $3.9 \times\left(1, \ldots, 2^{6}\right)$ \\
\hline $2008-159$ & 03-30-2007 & 0.73 & 0.29 & -2 & 1.85 & 2.34 & 2.3 & $7.0 \times\left(1, \ldots, 2^{8}\right)$ \\
\hline $2013+163$ & 03-30-2007 & 0.70 & 0.32 & -3 & 0.46 & 0.51 & 1.0 & $2.9 \times\left(1, \ldots, 2^{7}\right)$ \\
\hline $2021+317$ & $12-14-2004$ & 0.75 & 0.39 & 22 & 0.39 & 0.59 & 1.8 & $5.5 \times\left(1, \ldots, 2^{6}\right)$ \\
\hline $2029+121$ & $05-22-2003$ & 2.81 & 1.43 & 15 & 0.33 & 0.38 & 3.0 & $9.0 \times\left(1, \ldots, 2^{5}\right)$ \\
\hline $2037+511$ & $03-30-2007$ & 0.64 & 0.30 & 9 & 2.60 & 2.98 & 3.9 & $11.7 \times\left(1, \ldots, 2^{7}\right)$ \\
\hline $2048+312$ & 03-30-2007 & 0.80 & 0.33 & 19 & 0.22 & 0.29 & 1.5 & $4.5 \times\left(1, \ldots, 2^{5}\right)$ \\
\hline $2054-377$ & 05-22-2003 & 1.32 & 0.31 & -23 & 0.43 & 0.50 & 4.4 & $11.5 \times\left(1, \ldots, 2^{5}\right)$ \\
\hline $2106-413$ & $05-22-2003$ & 9.82 & 1.24 & 3 & 0.88 & 0.82 & 4.5 & $14.0 \times\left(1, \ldots, 2^{5}\right)$ \\
\hline $2113+293$ & 03-30-2007 & 0.74 & 0.31 & 11 & 0.64 & 0.79 & 1.6 & $4.8 \times\left(1, \ldots, 2^{7}\right)$ \\
\hline $2121+053$ & $12-14-2004$ & 0.72 & 0.29 & -2 & 1.61 & 2.60 & 1.9 & $5.6 \times\left(1, \ldots, 2^{8}\right)$ \\
\hline $2126-158$ & 03-30-2007 & 0.75 & 0.29 & -3 & 0.62 & 0.84 & 1.6 & $4.7 \times\left(1, \ldots, 2^{7}\right)$ \\
\hline $2128-123$ & 03-30-2007 & 0.74 & 0.29 & -4 & 0.53 & 1.88 & 4.1 & $12.2 \times\left(1, \ldots, 2^{5}\right)$ \\
\hline $2131-021$ & $12-14-2004$ & 0.79 & 0.36 & -1 & 1.35 & 2.23 & 2.6 & $7.8 \times\left(1, \ldots, 2^{7}\right)$ \\
\hline $2136+141$ & $03-30-2007$ & 0.73 & 0.31 & 3 & 0.74 & 1.55 & 3.3 & $10.0 \times\left(1, \ldots, 2^{6}\right)$ \\
\hline $2143-156$ & 03-30-2007 & 0.79 & 0.30 & -7 & 0.40 & 0.43 & 1.1 & $3.3 \times\left(1, \ldots, 2^{6}\right)$ \\
\hline $2145+067$ & $12-14-2004$ & 0.83 & 0.37 & 17 & 6.03 & 8.64 & 9.9 & $29.8 \times\left(1, \ldots, 2^{7}\right)$ \\
\hline $2150+173$ & 03-30-2007 & 0.74 & 0.32 & 8 & 0.31 & 0.60 & 1.4 & $4.1 \times\left(1, \ldots, 2^{6}\right)$ \\
\hline $2200+420$ & $12-14-2004$ & 0.63 & 0.34 & 17 & 3.37 & 3.98 & 2.9 & $8.8 \times\left(1, \ldots, 2^{8}\right)$ \\
\hline $2201+171$ & 03-30-2007 & 0.72 & 0.30 & 6 & 0.97 & 1.22 & 1.6 & $4.8 \times\left(1, \ldots, 2^{7}\right)$ \\
\hline $2209+236$ & $03-30-2007$ & 0.73 & 0.31 & 13 & 0.66 & 0.82 & 1.8 & $5.5 \times\left(1, \ldots, 2^{6}\right)$ \\
\hline $2214+350$ & 03-30-2007 & 0.98 & 0.45 & 12 & 0.25 & 0.29 & 1.5 & $4.4 \times\left(1, \ldots, 2^{5}\right)$ \\
\hline $2227-088$ & 03-30-2007 & 0.86 & 0.31 & -9 & 2.24 & 2.36 & 2.2 & $6.6 \times\left(1, \ldots, 2^{8}\right)$ \\
\hline $2239+096$ & 03-30-2007 & 0.72 & 0.34 & -2 & 0.58 & 0.67 & 1.3 & $3.9 \times\left(1, \ldots, 2^{7}\right)$ \\
\hline $2251+158$ & 03-30-2007 & 0.73 & 0.31 & 8 & 2.15 & 4.30 & 7.0 & $19.5 \times\left(1, \ldots, 2^{6}\right)$ \\
\hline $2254+024$ & 03-30-2007 & 0.84 & 0.34 & -10 & 0.37 & 0.39 & 1.2 & $3.6 \times\left(1, \ldots, 2^{6}\right)$ \\
\hline $2255-282$ & 03-30-2007 & 0.92 & 0.30 & -7 & 1.58 & 1.82 & 3.6 & $10.2 \times\left(1, \ldots, 2^{7}\right)$ \\
\hline $2306+095$ & $05-22-2003$ & 1.45 & 0.33 & -19 & 0.26 & 0.27 & 3.3 & $9.8 \times\left(1, \ldots, 2^{4}\right)$ \\
\hline $2309+454$ & 03-30-2007 & 0.81 & 0.38 & 5 & 0.19 & 0.26 & 1.1 & $3.3 \times\left(1, \ldots, 2^{5}\right)$ \\
\hline $2318+049$ & $12-14-2004$ & 0.89 & 0.42 & -8 & 0.45 & 0.57 & 1.5 & $4.4 \times\left(1, \ldots, 2^{6}\right)$ \\
\hline $2325+093$ & 03-30-2007 & 0.71 & 0.32 & -3 & 1.33 & 1.36 & 1.3 & $3.8 \times\left(1, \ldots, 2^{8}\right)$ \\
\hline $2335-027$ & 03-30-2007 & 0.90 & 0.32 & -10 & 0.57 & 0.74 & 1.4 & $4.3 \times\left(1, \ldots, 2^{7}\right)$ \\
\hline $2344+092$ & 03-30-2007 & 0.74 & 0.31 & 2 & 0.56 & 0.88 & 1.5 & $4.4 \times\left(1, \ldots, 2^{6}\right)$ \\
\hline $2346+385$ & 03-30-2007 & 0.77 & 0.33 & 25 & 0.37 & 0.55 & 1.5 & $4.6 \times\left(1, \ldots, 2^{6}\right)$ \\
\hline
\end{tabular}


TABLE 2 - Continued

\begin{tabular}{|c|c|c|c|c|c|c|c|c|}
\hline \multirow[b]{2}{*}{ Source } & \multirow[b]{2}{*}{$\begin{array}{c}\text { Observation } \\
\text { Session }\end{array}$} & \multicolumn{3}{|c|}{ Beam $^{a}$} & \multicolumn{3}{|c|}{ Flux Density } & \multirow[b]{2}{*}{$\begin{array}{l}\text { Contour Levels }{ }^{\mathrm{d}} \\
\left(\mathrm{mJy} \mathrm{bm}^{-1}\right)\end{array}$} \\
\hline & & $\begin{array}{c}a \\
(\mathrm{mas})\end{array}$ & $\begin{array}{c}b \\
\text { (mas) }\end{array}$ & $\begin{array}{c}\phi \\
\left({ }^{\circ}\right)\end{array}$ & $\begin{array}{c}S_{\text {peak }} \\
\left(\mathrm{Jy} \mathrm{bm}^{-1}\right)\end{array}$ & $\begin{array}{c}S_{\text {total }^{\mathrm{b}}} \\
\text { (Jy) }\end{array}$ & $\begin{array}{c}S_{\mathrm{rms}}^{\mathrm{c}} \\
\left(\mathrm{mJy} \mathrm{bm}^{-1}\right)\end{array}$ & \\
\hline $2353+816$ & & 0.43 & 0.34 & 61 & 0.84 & 0.90 & 1.3 & $4.0 \times\left(1, \ldots, 2^{7}\right)$ \\
\hline $2355-106$ & $03-30-2007$ & 0.83 & 0.31 & -7 & 0.58 & 0.75 & 1.5 & $4.6 \times\left(1, \ldots, 2^{6}\right)$ \\
\hline $2357-318$ & $03-30-2007$ & 1.35 & 0.35 & -18 & 0.50 & 0.62 & 4.1 & $12.3 \times\left(1, \ldots, 2^{5}\right)$ \\
\hline
\end{tabular}

a The restoring beam is an elliptical Gaussian with FWHM major axis $a$ and minor axis $b$, with major axis in position angle $\phi$ (measured north through east).

b The total image flux defined by the sum of the CLEAN components. In some cases this may be slightly less than the peak flux density due to the presence of components with negative amplitude in the CLEAN model.

c The root mean square (rms) of the residuals of the final hybrid image.

$\mathrm{d}$ Contours levels are represented by the geometric series $1, \ldots, 2^{n}$, e.g. for $n=5$ the contour levels would be $\pm 1,2,4,8,16,32$. 
TABLE 3

Parameters of Naturally Weighted Images at $43 \mathrm{GHz}$

\begin{tabular}{|c|c|c|c|c|c|c|c|c|}
\hline \multirow[b]{2}{*}{ Source } & \multirow[b]{2}{*}{$\begin{array}{l}\text { Observation } \\
\text { Epoch }\end{array}$} & \multicolumn{3}{|c|}{ Beam $^{\mathrm{a}}$} & \multicolumn{3}{|c|}{ Flux Density } & \multirow[b]{2}{*}{$\begin{array}{l}\text { Contour Levels }{ }^{\mathrm{d}} \\
\left(\mathrm{mJy} \mathrm{bm}^{-1}\right)\end{array}$} \\
\hline & & $\begin{array}{c}a \\
(\mathrm{mas})\end{array}$ & $\begin{array}{c}b \\
(\text { mas })\end{array}$ & $\begin{array}{c}\phi \\
\left(^{\circ}\right)\end{array}$ & $\begin{array}{c}S_{\text {peak }} \\
\left(\mathrm{Jy} \mathrm{bm}^{-1}\right)\end{array}$ & $\begin{array}{c}S_{\text {total }^{b}} \\
(\mathrm{Jy})\end{array}$ & $\begin{array}{c}\left.S_{\mathrm{rms}} \mathrm{c}^{-1}\right) \\
\left(\mathrm{mJy} \mathrm{bm}^{-1}\right)\end{array}$ & \\
\hline $0007+106$ & 09-13-2003 & 0.38 & 0.19 & 0 & 3.54 & 3.66 & 5.6 & $16.8 \times\left(1, \ldots, 2^{7}\right)$ \\
\hline $0009+081$ & $09-13-2003$ & 0.54 & 0.25 & -17 & 0.72 & 0.92 & 4.3 & $12.9 \times\left(1, \ldots, 2^{5}\right)$ \\
\hline $0016+731$ & $08-25-2002$ & 0.25 & 0.21 & 27 & 0.61 & 0.77 & 2.6 & $7.7 \times\left(1, \ldots, 2^{6}\right)$ \\
\hline $0017+200$ & $09-13-2003$ & 0.40 & 0.20 & -3 & 0.95 & 1.13 & 4.6 & $13.8 \times\left(1, \ldots, 2^{6}\right)$ \\
\hline $0046+316$ & $09-13-2003$ & 0.61 & 0.36 & 4 & 0.74 & 0.80 & 4.3 & $11.9 \times\left(1, \ldots, 2^{5}\right)$ \\
\hline $0048-097$ & 09-13-2003 & 0.44 & 0.17 & 7 & 1.13 & 1.14 & 7.4 & $22.2 \times\left(1, \ldots, 2^{5}\right)$ \\
\hline $0059+581$ & $09-13-2003$ & 0.40 & 0.16 & 33 & 2.89 & 4.09 & 8.7 & $26.2 \times\left(1, \ldots, 2^{6}\right)$ \\
\hline $0119+115$ & $09-13-2003$ & 0.61 & 0.25 & 23 & 1.23 & 1.45 & 4.5 & $13.4 \times\left(1, \ldots, 2^{6}\right)$ \\
\hline $0122-003$ & $09-13-2003$ & 0.47 & 0.21 & -5 & 0.74 & 1.03 & 6.0 & $17.9 \times\left(1, \ldots, 2^{5}\right)$ \\
\hline $0133+476$ & $08-25-2002$ & 0.39 & 0.17 & 19 & 2.84 & 3.39 & 3.3 & $10.0 \times\left(1, \ldots, 2^{8}\right)$ \\
\hline $0138-097$ & $09-13-2003$ & 1.90 & 0.27 & 34 & 0.69 & 0.64 & 4.2 & $12.6 \times\left(1, \ldots, 2^{5}\right)$ \\
\hline $0149+218$ & 09-13-2003 & 0.40 & 0.19 & 21 & 1.65 & 1.91 & 8.6 & $25.7 \times\left(1, \ldots, 2^{5}\right)$ \\
\hline $0202+149$ & $09-13-2003$ & 1.52 & 0.37 & -11 & 0.94 & 1.01 & 4.3 & $13.9 \times\left(1, \ldots, 2^{6}\right)$ \\
\hline $0212+735$ & $08-25-2002$ & 0.47 & 0.36 & 53 & 0.37 & 0.81 & 4.2 & $12.5 \times\left(1, \ldots, 2^{4}\right)$ \\
\hline $0224+671$ & $09-13-2003$ & 0.46 & 0.16 & 8 & 0.97 & $\begin{array}{l}0.01 \\
1.26\end{array}$ & 6.7 & $18.1 \times\left(1, \ldots, 2^{5}\right)$ \\
\hline $0229+131$ & $09-13-2003$ & 0.55 & 0.30 & 13 & 0.79 & 0.95 & 6.0 & $17.3 \times\left(1, \ldots, 2^{5}\right)$ \\
\hline $0234+285$ & $09-13-2003$ & 0.36 & 0.17 & -5 & 1.59 & 2.62 & 14.2 & $42.5 \times\left(1, \ldots, 2^{5}\right)$ \\
\hline $0235+164$ & $08-25-2002$ & 0.40 & 0.17 & 2 & 0.85 & 1.23 & 2.6 & $7.9 \times\left(1, \ldots, 2^{6}\right)$ \\
\hline $0237-027$ & $12-26-2002$ & 0.84 & 0.16 & -16 & 0.38 & 0.43 & 2.0 & $5.7 \times\left(1, \ldots, 2^{6}\right)$ \\
\hline $0237+040$ & $12-26-2002$ & 0.46 & 0.16 & -7 & 0.46 & 0.52 & 2.3 & $6.8 \times\left(1, \ldots, 2^{6}\right)$ \\
\hline $0239+108$ & $09-13-2003$ & 0.42 & 0.18 & -9 & 0.78 & 0.90 & 6.5 & $19.5 \times\left(1, \ldots, 2^{5}\right)$ \\
\hline $0241+622$ & $09-13-2003$ & 0.27 & 0.21 & -57 & 1.01 & 1.12 & 5.2 & $15.6 \times\left(1, \ldots, 2^{6}\right)$ \\
\hline $0300+470$ & $09-13-2003$ & 0.43 & 0.18 & 8 & 0.90 & 1.20 & 5.4 & $16.3 \times\left(1, \ldots, 2^{5}\right)$ \\
\hline $0306+102$ & 09-13-2003 & 0.38 & 0.16 & 5 & 1.66 & 1.82 & 7.6 & $22.8 \times\left(1, \ldots, 2^{6}\right)$ \\
\hline $0333+321$ & 09-13-2003 & 0.38 & 0.17 & 8 & 2.26 & 3.23 & 7.3 & $21.9 \times\left(1, \ldots, 2^{6}\right)$ \\
\hline $0336-019$ & $09-13-2003$ & 0.43 & 0.16 & -6 & 1.56 & 2.55 & 7.0 & $21.0 \times\left(1, \ldots, 2^{6}\right)$ \\
\hline $0340+362$ & $09-13-2003$ & 0.46 & 0.16 & 0 & 0.46 & 0.41 & 5.2 & $15.6 \times\left(1, \ldots, 2^{4}\right)$ \\
\hline $0345+460$ & $09-13-2003$ & 0.84 & 0.26 & -25 & 0.49 & 0.58 & 4.5 & $13.6 \times\left(1, \ldots, 2^{5}\right)$ \\
\hline $0346-279$ & $09-13-2003$ & 0.55 & 0.16 & -15 & 0.71 & 0.72 & 8.2 & $24.7 \times\left(1, \ldots, 2^{4}\right)$ \\
\hline $0350+465$ & $09-13-2003$ & 0.30 & 0.19 & 13 & 0.80 & 0.80 & 5.1 & $15.2 \times\left(1, \ldots, 2^{5}\right)$ \\
\hline $0358+040$ & 09-13-2003 & 0.41 & 0.18 & -1 & 1.11 & 1.09 & 3.6 & $10.8 \times\left(1, \ldots, 2^{6}\right)$ \\
\hline $0400+258$ & 09-13-2003 & 0.47 & 0.19 & -14 & 0.81 & 1.01 & 5.2 & $15.6 \times\left(1, \ldots, 2^{5}\right)$ \\
\hline $0446+112$ & $08-25-2002$ & 0.40 & 0.16 & 1 & 1.10 & 1.47 & 2.1 & $6.2 \times\left(1, \ldots, 2^{7}\right)$ \\
\hline $0454-234$ & $09-13-2003$ & 0.40 & 0.16 & -4 & 1.43 & 2.09 & 9.6 & $28.9 \times\left(1, \ldots, 2^{5}\right)$ \\
\hline $0458-020$ & $09-13-2003$ & 0.43 & 0.17 & -5 & 1.42 & 1.59 & 5.4 & $16.1 \times\left(1, \ldots, 2^{6}\right)$ \\
\hline $0506+056$ & $09-13-2003$ & 0.46 & 0.19 & -3 & 0.58 & 0.49 & 6.9 & $20.6 \times\left(1, \ldots, 2^{4}\right)$ \\
\hline $0524+034$ & 09-13-2003 & 0.42 & 0.17 & -2 & 0.99 & 1.16 & 5.0 & $14.0 \times\left(1, \ldots, 2^{6}\right)$ \\
\hline $0528+134$ & $08-25-2002$ & 0.38 & 0.19 & 1 & 0.79 & 1.51 & 3.2 & $9.6 \times\left(1, \ldots, 2^{6}\right)$ \\
\hline $0552+398$ & $08-25-2002$ & 0.43 & 0.18 & 23 & 0.90 & 1.47 & 3.8 & $11.3 \times\left(1, \ldots, 2^{6}\right)$ \\
\hline $0556+238$ & $09-13-2003$ & 0.50 & 0.26 & -12 & 0.43 & 0.39 & 6.0 & $18.1 \times\left(1, \ldots, 2^{4}\right)$ \\
\hline $0607-157$ & $08-25-2002$ & 0.41 & 0.17 & -2 & 1.82 & 2.70 & 5.0 & $15.0 \times\left(1, \ldots, 2^{6}\right)$ \\
\hline $0642+449$ & $08-25-2002$ & 0.29 & 0.19 & 1 & 0.97 & 1.83 & 3.0 & $9.0 \times\left(1, \ldots, 2^{6}\right)$ \\
\hline $0646-306$ & 09-13-2003 & 0.49 & 0.18 & -15 & 0.64 & 0.75 & 10.1 & $30.2 \times\left(1, \ldots, 2^{4}\right)$ \\
\hline $0648-165$ & $09-13-2003$ & 0.47 & 0.18 & 4 & 2.09 & 2.73 & 11.6 & $34.8 \times\left(1, \ldots, 2^{5}\right)$ \\
\hline $0707+476$ & $12-26-2002$ & 0.91 & 0.18 & -17 & 0.25 & 0.26 & 0.5 & $1.7 \times\left(1, \ldots, 2^{7}\right)$ \\
\hline $0722+145$ & $12-26-2002$ & 0.45 & 0.18 & -7 & 0.48 & 0.60 & 1.7 & $5.0 \times\left(1, \ldots, 2^{6}\right)$ \\
\hline $0727-115$ & $09-13-2003$ & 0.54 & 0.18 & -11 & 2.22 & 3.01 & 12.3 & $37.0 \times\left(1, \ldots, 2^{5}\right)$ \\
\hline $0735+178$ & $09-13-2003$ & 0.50 & 0.20 & -15 & 0.71 & 1.05 & 3.8 & $11.5 \times\left(1, \ldots, 2^{5}\right)$ \\
\hline $0743-006$ & 08-25-2002 & 0.45 & 0.20 & -3 & 0.32 & 0.59 & 2.9 & $8.6 \times\left(1, \ldots, 2^{5}\right)$ \\
\hline $0745+241$ & $09-13-2003$ & 0.44 & 0.21 & -21 & 0.79 & 0.97 & 5.0 & $15.0 \times\left(1, \ldots, 2^{5}\right)$ \\
\hline $0746+483$ & $09-13-2003$ & 0.51 & 0.23 & -19 & 0.55 & 0.60 & 4.3 & $12.9 \times\left(1, \ldots, 2^{5}\right)$ \\
\hline $0748+126$ & $08-25-2002$ & 0.39 & 0.18 & -2 & 1.26 & 1.67 & 2.7 & $8.2 \times\left(1, \ldots, 2^{7}\right)$ \\
\hline $0749+540$ & $09-13-2003$ & 0.34 & 0.22 & -20 & 0.69 & 0.83 & 3.1 & $9.4 \times\left(1, \ldots, 2^{6}\right)$ \\
\hline $0754+100$ & $09-13-2003$ & 0.41 & 0.17 & -4 & 1.71 & 2.22 & 4.7 & $14.0 \times\left(1, \ldots, 2^{6}\right)$ \\
\hline $0804+499$ & 09-13-2003 & 0.30 & 0.20 & -20 & 0.78 & 0.80 & 3.0 & $9.1 \times\left(1, \ldots, 2^{6}\right)$ \\
\hline $0805+410$ & $08-25-2002$ & 0.42 & 0.19 & 23 & 0.38 & 0.45 & 2.2 & $6.3 \times\left(1, \ldots, 2^{5}\right)$ \\
\hline $0808+019$ & $09-13-2003$ & 0.50 & 0.22 & -8 & 0.69 & 0.70 & 3.5 & $10.4 \times\left(1, \ldots, 2^{6}\right)$ \\
\hline $0814+425$ & $08-25-2002$ & 0.31 & 0.20 & -7 & 0.36 & 0.39 & 2.6 & $7.8 \times\left(1, \ldots, 2^{5}\right)$ \\
\hline $0820+560$ & $08-25-2002$ & 0.25 & 0.22 & 66 & 0.44 & 0.56 & 3.0 & $9.0 \times\left(1, \ldots, 2^{5}\right)$ \\
\hline $0823+033$ & $08-25-2002$ & 0.41 & 0.18 & -5 & 1.08 & 1.17 & 2.4 & $7.1 \times\left(1, \ldots, 2^{7}\right)$ \\
\hline $0838+133$ & $09-13-2003$ & 0.42 & 0.19 & -2 & 1.30 & 2.22 & 5.2 & $15.7 \times\left(1, \ldots, 2^{6}\right)$ \\
\hline $0851+202$ & 08-25-2002 & 0.36 & 0.18 & -7 & 2.33 & 2.90 & 3.8 & $11.3 \times\left(1, \ldots, 2^{7}\right)$ \\
\hline $0917+624$ & $08-25-2002$ & 1.24 & 0.58 & -70 & 0.34 & 0.39 & 2.1 & $6.4 \times\left(1, \ldots, 2^{5}\right)$ \\
\hline $0923+392$ & $08-25-2002$ & 0.32 & 0.18 & 3 & 0.83 & 3.90 & 4.1 & $12.2 \times\left(1, \ldots, 2^{6}\right)$ \\
\hline $0953+254$ & $09-13-2003$ & 0.36 & 0.18 & -6 & 1.32 & 1.47 & 3.7 & $11.0 \times\left(1, \ldots, 2^{6}\right)$ \\
\hline $0955+476$ & $12-26-2002$ & 0.31 & 0.22 & -19 & 0.50 & 0.80 & 4.0 & $11.9 \times\left(1, \ldots, 2^{5}\right)$ \\
\hline $1034-293$ & $09-13-2003$ & 0.90 & 0.18 & -24 & 1.18 & 1.29 & 9.9 & $29.6 \times\left(1, \ldots, 2^{5}\right)$ \\
\hline
\end{tabular}


TABLE 3 - Continued

\begin{tabular}{|c|c|c|c|c|c|c|c|c|}
\hline \multirow[b]{2}{*}{ Source } & \multirow[b]{2}{*}{$\begin{array}{c}\text { Observation } \\
\text { Epoch }\end{array}$} & \multicolumn{3}{|c|}{ Beam $^{\mathrm{a}}$} & \multicolumn{3}{|c|}{ Flux Density } & \multirow[b]{2}{*}{$\begin{array}{l}\text { Contour Levels } \\
\left(\mathrm{mJy} \mathrm{bm}^{\mathrm{d}}\right)\end{array}$} \\
\hline & & $\begin{array}{c}a \\
(\mathrm{mas})\end{array}$ & $\begin{array}{c}b \\
(\mathrm{mas})\end{array}$ & $\begin{array}{c}\phi \\
\left({ }^{\circ}\right)\end{array}$ & $\begin{array}{c}S_{\text {peak }} \\
\left(\mathrm{Jy} \mathrm{bm}^{-1}\right)\end{array}$ & $\begin{array}{c}S_{\text {total }^{\mathrm{b}}} \\
(\mathrm{Jy})\end{array}$ & $\begin{array}{c}S_{\mathrm{rms}}{ }^{\mathrm{c}} \\
\left(\mathrm{mJy} \mathrm{bm}^{-1}\right)\end{array}$ & \\
\hline $1039+811$ & $12-26-2002$ & 0.57 & 0.18 & 75 & 0.45 & 0.48 & 3.4 & $9.1 \times\left(1, \ldots, 2^{5}\right)$ \\
\hline $1045-188$ & $09-13-2003$ & 0.82 & 0.19 & -18 & 0.82 & 1.02 & 4.2 & $12.7 \times\left(1, \ldots, 2^{6}\right)$ \\
\hline $1049+215$ & $12-26-2002$ & 0.46 & 0.18 & -15 & 0.37 & 0.42 & 2.2 & $6.6 \times\left(1, \ldots, 2^{5}\right)$ \\
\hline $1053+815$ & $09-13-2003$ & 0.23 & 0.21 & -33 & 1.43 & 1.55 & 2.2 & $6.7 \times\left(1, \ldots, 2^{7}\right)$ \\
\hline $1055+018$ & $12-26-2002$ & 0.47 & 0.17 & -7 & 1.54 & 2.37 & 4.1 & $11.5 \times\left(1, \ldots, 2^{7}\right)$ \\
\hline $1124-186$ & 09-13-2003 & 0.40 & 0.15 & -1 & 1.47 & 1.76 & 5.5 & $16.6 \times\left(1, \ldots, 2^{6}\right)$ \\
\hline $1128+385$ & $12-26-2002$ & 0.32 & 0.17 & -8 & 0.65 & 0.91 & 2.7 & $8.0 \times\left(1, \ldots, 2^{6}\right)$ \\
\hline $1144+402$ & $08-25-2002$ & 0.54 & 0.21 & -25 & 0.33 & 0.39 & 2.3 & $6.8 \times\left(1, \ldots, 2^{5}\right)$ \\
\hline $1156+295$ & $12-26-2002$ & 0.38 & 0.17 & -7 & 1.18 & 1.43 & 3.2 & $9.6 \times\left(1, \ldots, 2^{6}\right)$ \\
\hline $1236+077$ & $12-26-2002$ & 0.80 & 0.20 & -16 & 0.39 & 0.46 & 2.5 & $7.4 \times\left(1, \ldots, 2^{5}\right)$ \\
\hline $1243-072$ & $12-26-2002$ & 0.55 & 0.16 & -11 & 0.41 & 0.47 & 1.7 & $5.2 \times\left(1, \ldots, 2^{6}\right)$ \\
\hline $1256-220$ & 09-13-2003 & 0.50 & 0.18 & -7 & 1.05 & 1.00 & 7.1 & $21.2 \times\left(1, \ldots, 2^{5}\right)$ \\
\hline $1302-102$ & $12-26-2002$ & 0.51 & 0.16 & -8 & 0.44 & 0.52 & 2.1 & $6.3 \times\left(1, \ldots, 2^{6}\right)$ \\
\hline $1308+326$ & $09-13-2003$ & 0.37 & 0.17 & 4 & 2.94 & 3.82 & 5.6 & $16.8 \times\left(1, \ldots, 2^{7}\right)$ \\
\hline $1313-333$ & $08-25-2002$ & 0.52 & 0.20 & -5 & 0.64 & 0.79 & 4.0 & $12.0 \times\left(1, \ldots, 2^{5}\right)$ \\
\hline $1324+224$ & $08-25-2002$ & 0.34 & 0.18 & -3 & 0.39 & 0.48 & 1.9 & $5.8 \times\left(1, \ldots, 2^{6}\right)$ \\
\hline $1334-127$ & $08-25-2002$ & 0.46 & 0.17 & -6 & 3.87 & 5.05 & 4.4 & $13.2 \times\left(1, \ldots, 2^{8}\right)$ \\
\hline $1354-152$ & $12-26-2002$ & 0.54 & 0.16 & -11 & 0.54 & 0.68 & 2.3 & $6.4 \times\left(1, \ldots, 2^{6}\right)$ \\
\hline $1357+769$ & $12-26-2002$ & 0.34 & 0.16 & -70 & 0.63 & 0.67 & 3.5 & $10.5 \times\left(1, \ldots, 2^{5}\right)$ \\
\hline $1406-076$ & $12-26-2002$ & 0.45 & 0.16 & -6 & 0.74 & 0.97 & 2.2 & $6.6 \times\left(1, \ldots, 2^{6}\right)$ \\
\hline $1502+036$ & $12-26-2002$ & 0.48 & 0.17 & -10 & 0.39 & 0.43 & 1.7 & $5.1 \times\left(1, \ldots, 2^{6}\right)$ \\
\hline $1502+106$ & 09-13-2003 & 0.40 & 0.16 & 1 & 1.27 & 2.30 & 3.8 & $11.3 \times\left(1, \ldots, 2^{6}\right)$ \\
\hline $1505+428$ & $09-13-2003$ & 0.40 & 0.24 & 12 & 0.70 & 0.90 & 2.7 & $8.2 \times\left(1, \ldots, 2^{6}\right)$ \\
\hline $1511-100$ & $12-26-2002$ & 0.47 & 0.16 & -8 & 0.51 & 0.61 & 2.6 & $7.4 \times\left(1, \ldots, 2^{6}\right)$ \\
\hline $1514+004$ & $09-13-2003$ & 0.49 & 0.17 & -7 & 0.86 & 1.17 & 3.6 & $10.8 \times\left(1, \ldots, 2^{6}\right)$ \\
\hline $1546+027$ & $08-25-2002$ & 0.41 & 0.16 & -2 & 0.95 & 1.32 & 3.6 & $10.8 \times\left(1, \ldots, 2^{6}\right)$ \\
\hline $1606+106$ & $08-25-2002$ & 0.41 & 0.17 & -7 & 0.76 & 1.36 & 2.7 & $8.1 \times\left(1, \ldots, 2^{6}\right)$ \\
\hline $1611+343$ & $12-26-2002$ & 0.39 & 0.21 & -2 & 1.09 & 1.37 & 9.4 & $26.4 \times\left(1, \ldots, 2^{5}\right)$ \\
\hline $1617+229$ & $09-13-2003$ & 1.18 & 0.45 & 1 & 0.61 & 0.68 & 3.2 & $9.6 \times\left(1, \ldots, 2^{5}\right)$ \\
\hline $1637+574$ & $08-25-2002$ & 0.30 & 0.19 & 15 & 0.81 & 1.37 & 3.4 & $10.3 \times\left(1, \ldots, 2^{6}\right)$ \\
\hline $1638+398$ & $08-25-2002$ & 0.35 & 0.20 & -6 & 0.43 & 0.57 & 2.8 & $8.3 \times\left(1, \ldots, 2^{5}\right)$ \\
\hline $1705+018$ & $12-26-2002$ & 0.50 & 0.17 & -6 & 0.41 & 0.41 & 2.3 & $6.3 \times\left(1, \ldots, 2^{6}\right)$ \\
\hline $1717+178$ & $12-26-2002$ & 0.42 & 0.16 & -2 & 0.37 & 0.45 & 2.5 & $6.9 \times\left(1, \ldots, 2^{5}\right)$ \\
\hline $1726+455$ & $09-13-2003$ & 0.31 & 0.17 & 15 & 1.67 & 1.78 & 2.8 & $8.4 \times\left(1, \ldots, 2^{7}\right)$ \\
\hline $1730-130$ & $09-13-2003$ & 0.43 & 0.17 & -1 & 3.89 & 5.59 & 11.7 & $35.0 \times\left(1, \ldots, 2^{6}\right)$ \\
\hline $1732+389$ & $08-25-2002$ & 0.33 & 0.18 & -6 & 0.47 & 0.68 & 2.5 & $7.6 \times\left(1, \ldots, 2^{5}\right)$ \\
\hline $1738+476$ & $12-26-2002$ & 0.46 & 0.16 & 3 & 0.34 & 0.39 & 2.1 & $6.4 \times\left(1, \ldots, 2^{5}\right)$ \\
\hline $1741-038$ & $09-13-2003$ & 0.41 & 0.16 & 0 & 5.51 & 8.49 & 18.9 & $56.6 \times\left(1, \ldots, 2^{6}\right)$ \\
\hline $1742-078$ & 09-13-2003 & 0.43 & 0.17 & 0 & 0.82 & 1.03 & 4.0 & $11.9 \times\left(1, \ldots, 2^{6}\right)$ \\
\hline $1749+096$ & $08-25-2002$ & 0.41 & 0.17 & -4 & 2.92 & 3.25 & 3.2 & $9.6 \times\left(1, \ldots, 2^{8}\right)$ \\
\hline $1751+288$ & $12-26-2002$ & 0.37 & 0.16 & -11 & 1.35 & 1.50 & 4.7 & $14.2 \times\left(1, \ldots, 2^{6}\right)$ \\
\hline $1803+784$ & $08-25-2002$ & 0.23 & 0.21 & -8 & 1.03 & 1.52 & 3.0 & $9.0 \times\left(1, \ldots, 2^{6}\right)$ \\
\hline $1849+670$ & $08-25-2002$ & 0.26 & 0.20 & 10 & 1.05 & 1.32 & 2.9 & $8.7 \times\left(1, \ldots, 2^{6}\right)$ \\
\hline $1901+319$ & 09-13-2003 & 0.42 & 0.23 & -22 & 0.88 & 1.01 & 4.6 & $13.0 \times\left(1, \ldots, 2^{6}\right)$ \\
\hline $1921-293$ & 09-13-2003 & 0.41 & 0.15 & -7 & 14.04 & 18.40 & 33.2 & $96.2 \times\left(1, \ldots, 2^{7}\right)$ \\
\hline $1936-155$ & 09-13-2003 & 0.68 & 0.20 & -9 & 0.86 & 0.81 & 10.3 & $30.8 \times\left(1, \ldots, 2^{4}\right)$ \\
\hline $1958-179$ & $12-26-2002$ & 0.42 & 0.15 & -4 & 0.87 & 0.94 & 2.7 & $8.1 \times\left(1, \ldots, 2^{6}\right)$ \\
\hline $2008-159$ & 09-13-2003 & 0.49 & 0.18 & 6 & 1.75 & 2.26 & 5.7 & $17.0 \times\left(1, \ldots, 2^{6}\right)$ \\
\hline $2021+317$ & 09-13-2003 & 0.42 & 0.21 & 15 & 0.71 & 0.67 & 7.1 & $21.4 \times\left(1, \ldots, 2^{5}\right)$ \\
\hline $2037+511$ & $12-26-2002$ & 0.40 & 0.17 & -22 & 0.76 & 1.08 & 3.7 & $10.8 \times\left(1, \ldots, 2^{6}\right)$ \\
\hline $2113+293$ & $12-26-2002$ & 0.75 & 0.17 & -23 & 0.28 & 0.31 & 2.2 & $6.5 \times\left(1, \ldots, 2^{5}\right)$ \\
\hline $2121+053$ & $08-25-2002$ & 0.42 & 0.18 & -5 & 1.44 & 1.72 & 3.3 & $9.8 \times\left(1, \ldots, 2^{7}\right)$ \\
\hline $2126-158$ & $12-26-2002$ & 1.24 & 0.41 & -4 & 0.42 & 0.50 & 5.6 & $15.6 \times\left(1, \ldots, 2^{4}\right)$ \\
\hline $2131-021$ & $08-25-2002$ & 0.43 & 0.17 & -5 & 0.59 & 1.16 & 3.4 & $10.1 \times\left(1, \ldots, 2^{5}\right)$ \\
\hline $2136+141$ & 09-13-2003 & 0.59 & 0.20 & -16 & 1.16 & 1.82 & 4.6 & $13.9 \times\left(1, \ldots, 2^{6}\right)$ \\
\hline $2145+067$ & $08-25-2002$ & 0.42 & 0.18 & -5 & 3.65 & 6.48 & 7.8 & $23.5 \times\left(1, \ldots, 2^{7}\right)$ \\
\hline $2150+173$ & $12-26-2002$ & 0.66 & 0.18 & -19 & 0.35 & 0.43 & 2.1 & $6.2 \times\left(1, \ldots, 2^{5}\right)$ \\
\hline $2200+420$ & $09-13-2003$ & 0.27 & 0.19 & 13 & 3.27 & 4.40 & 11.2 & $33.7 \times\left(1, \ldots, 2^{6}\right)$ \\
\hline $2227-088$ & 09-13-2003 & 0.40 & 0.15 & 0 & 5.11 & 5.27 & 11.4 & $34.1 \times\left(1, \ldots, 2^{7}\right)$ \\
\hline $2239+096$ & 09-13-2003 & 0.88 & 0.24 & -14 & 0.52 & 0.61 & 4.1 & $11.9 \times\left(1, \ldots, 2^{5}\right)$ \\
\hline $2255-282$ & 09-13-2003 & 0.41 & 0.15 & -3 & 2.77 & 4.59 & 14.8 & $42.9 \times\left(1, \ldots, 2^{6}\right)$ \\
\hline $2309+454$ & $09-13-2003$ & 0.72 & 0.27 & -17 & 0.51 & 0.60 & 4.9 & $12.3 \times\left(1, \ldots, 2^{5}\right)$ \\
\hline $2318+049$ & $08-25-2002$ & 0.42 & 0.18 & -4 & 0.58 & 0.71 & 2.9 & $8.7 \times\left(1, \ldots, 2^{6}\right)$ \\
\hline $2325+093$ & $09-13-2003$ & 0.38 & 0.17 & -2 & 1.54 & 1.66 & 5.6 & $16.4 \times\left(1, \ldots, 2^{6}\right)$ \\
\hline $2353+816$ & 09-13-2003 & 0.46 & 0.26 & 62 & 0.96 & 0.93 & 4.0 & $12.0 \times\left(1, \ldots, 2^{6}\right)$ \\
\hline
\end{tabular}


TABLE 3 - Continued

\begin{tabular}{|c|c|c|c|c|c|c|c|c|}
\hline \multirow[b]{2}{*}{ Source } & \multirow[b]{2}{*}{$\begin{array}{l}\text { Observation } \\
\text { Epoch }\end{array}$} & \multicolumn{3}{|c|}{ Beam $^{\mathrm{a}}$} & \multicolumn{3}{|c|}{$\begin{array}{l}\text { Flux Density } \\
\end{array}$} & \multirow[b]{2}{*}{$\begin{array}{l}\text { Contour Levels } \\
\left(\mathrm{mJy} \mathrm{bm}^{-1}\right)\end{array}$} \\
\hline & & $\begin{array}{c}a \\
\text { (mas) }\end{array}$ & $\begin{array}{c}b \\
(\mathrm{mas})\end{array}$ & $\begin{array}{c}\phi \\
\left({ }^{\circ}\right)\end{array}$ & $\begin{array}{c}S_{\text {peak }} \\
\left(\mathrm{Jy} \mathrm{bm}^{-1}\right)\end{array}$ & $\begin{array}{c}S_{\text {total }^{\mathrm{b}}} \\
(\mathrm{Jy})\end{array}$ & $\begin{array}{c}S_{\mathrm{rms}}{ }^{\mathrm{c}} \\
\left(\mathrm{mJy} \mathrm{bm}^{-1}\right)\end{array}$ & \\
\hline
\end{tabular}

a The restoring beam is an elliptical Gaussian with FWHM major axis $a$ and minor axis $b$, with major axis in position angle $\phi$ (measured north through east).

b The total image flux defined by the sum of the CLEAN components. In some cases this may be slightly less than the peak flux density due to the presence of components with negative amplitude in the CLEAN model.

c The root mean square ( $\mathrm{rms}$ ) of the residuals of the final hybrid image.

$\mathrm{d}$ Contours levels are represented by the geometric series $1, \ldots, 2^{n}$, e.g. for $n=5$ the contour levels would be $\pm 1,2,4,8,16,32$. 
TABLE 4

Source STRUCtURE INDICES.

\begin{tabular}{|c|c|c|c|c|c|c|c|c|c|}
\hline \multirow[b]{2}{*}{ Source } & \multicolumn{3}{|c|}{$\mathrm{X}$ band $(8.4 \mathrm{GHz})$} & \multicolumn{3}{|c|}{$\mathrm{K}$ band $(24 \mathrm{GHz})$} & \multicolumn{3}{|c|}{$\mathrm{Q}$ band $(43 \mathrm{GHz})$} \\
\hline & $N_{\text {session }}$ & $S I_{\min }$ & $S I_{\max }$ & $N_{\text {session }}$ & $S I_{\min }$ & $S I_{\max }$ & $N_{\text {session }}$ & $S I_{\min }$ & $S I_{\max }$ \\
\hline $0006+061$ & $\cdots$ & $\ldots$ & $\ldots$ & 1 & 1 & 1 & $\ldots$ & $\ldots$ & $\ldots$ \\
\hline $0007+106$ & 1 & 1 & 1 & 6 & 1 & 2 & 1 & 1 & 1 \\
\hline $0009+081$ & 1 & 1 & 1 & 6 & 1 & 2 & 1 & 1 & 1 \\
\hline $0016+731$ & 1 & 1 & 1 & 4 & 1 & 2 & 2 & 1 & 1 \\
\hline $0017+200$ & 1 & 2 & 2 & 6 & 1 & 2 & 1 & 1 & 1 \\
\hline $0019+058$ & $\cdots$ & $\ldots$ & $\cdots$ & 3 & 1 & 1 & $\ldots$ & $\ldots$ & $\ldots$ \\
\hline $0038-020$ & $\ldots$ & $\ldots$ & $\ldots$ & 1 & 1 & 1 & $\ldots$ & $\ldots$ & $\ldots$ \\
\hline $0046+316$ & 1 & 2 & 2 & 6 & 1 & 2 & 1 & 1 & 1 \\
\hline 0048-097 & 1 & 1 & 1 & 6 & 1 & 1 & 3 & 1 & 1 \\
\hline $0054+161$ & $\cdots$ & $\ldots$ & $\cdots$ & 1 & 1 & 1 & $\cdots$ & $\cdots$ & . \\
\hline $0059+581$ & $\ldots$ & $\ldots$ & $\ldots$ & 6 & 1 & 1 & 4 & 1 & 2 \\
\hline 0104-408 & $\cdots$ & $\ldots$ & $\ldots$ & 1 & 1 & 1 & $\ldots$ & $\ldots$ & $\ldots$ \\
\hline $0109+224$ & 1 & 2 & 2 & 4 & 1 & 1 & $\ldots$ & $\ldots$ & $\ldots$ \\
\hline $0110+495$ & $\ldots$ & $\ldots$ & $\ldots$ & 3 & 1 & 2 & $\ldots$ & $\ldots$ & $\ldots$ \\
\hline $0111+021$ & $\ldots$ & $\ldots$ & $\ldots$ & 3 & 2 & 2 & $\ldots$ & $\ldots$ & $\ldots$ \\
\hline $0119+041$ & $\ldots$ & $\ldots$ & $\ldots$ & 3 & 2 & 2 & $\ldots$ & $\ldots$ & $\ldots$ \\
\hline $0119+115$ & $\ldots$ & $\ldots$ & $\ldots$ & 3 & 1 & 3 & 2 & 1 & 2 \\
\hline $0119+247$ & $\ldots$ & $\ldots$ & $\ldots$ & 3 & 2 & 2 & $\ldots$ & $\ldots$ & $\ldots$ \\
\hline 0122-003 & $\ldots$ & $\ldots$ & $\ldots$ & 4 & 2 & 3 & 1 & 2 & 2 \\
\hline $0123+257$ & 1 & 2 & 2 & 4 & 1 & 1 & $\ldots$ & $\ldots$ & $\ldots$ \\
\hline $0127+084$ & $\ldots$ & $\ldots$ & $\ldots$ & 2 & 1 & 1 & $\ldots$ & $\ldots$ & $\ldots$ \\
\hline $0133+476$ & 1 & 1 & 1 & 5 & 1 & 1 & 2 & 1 & 1 \\
\hline 0138-097 & 1 & 3 & 3 & 3 & 1 & 2 & 1 & 2 & 2 \\
\hline $0149+218$ & 2 & 2 & 2 & 10 & 1 & 2 & 4 & 1 & 1 \\
\hline $0202+149$ & 1 & 3 & 3 & 6 & 2 & 3 & 2 & 1 & 2 \\
\hline $0202+319$ & 1 & 1 & 1 & 3 & 1 & 1 & $\ldots$ & $\ldots$ & $\cdots$ \\
\hline $0212+735$ & 2 & 2 & 2 & 8 & 2 & 3 & 2 & 2 & 3 \\
\hline $0213-026$ & $\cdots$ & $\ldots$ & $\ldots$ & 3 & 2 & 2 & $\ldots$ & $\ldots$ & $\ldots$ \\
\hline $0220-349$ & $\ldots$ & $\ldots$ & $\ldots$ & 1 & 3 & 3 & $\ldots$ & $\ldots$ & $\ldots$ \\
\hline $0221+067$ & 2 & 2 & 2 & 6 & 1 & 2 & $\ldots$ & $\ldots$ & $\ldots$ \\
\hline $0222+185$ & $\ldots$ & $\cdots$ & $\ldots$ & 1 & 1 & 1 & $\ldots$ & $\ldots$ & $\ldots$ \\
\hline $0224+671$ & 1 & 3 & 3 & 6 & 1 & 3 & 3 & 2 & 2 \\
\hline $0229+131$ & $\ldots$ & $\cdots$ & $\ldots$ & 3 & 1 & 3 & 2 & 1 & 1 \\
\hline $0234+285$ & 1 & 1 & 1 & 6 & 1 & 3 & 2 & 2 & 2 \\
\hline $0235+164$ & 2 & 1 & 1 & 7 & 1 & 1 & 2 & 1 & 1 \\
\hline $0237-027$ & $\ldots$ & $\ldots$ & $\ldots$ & 3 & 1 & 2 & 1 & 1 & 1 \\
\hline $0237+040$ & $\ldots$ & $\ldots$ & $\ldots$ & 3 & 1 & 1 & 1 & 1 & 1 \\
\hline $0239+108$ & 1 & 3 & 3 & 6 & 1 & 2 & 2 & 1 & 1 \\
\hline $0241+622$ & 1 & 3 & 3 & 6 & 2 & 2 & 3 & 1 & 2 \\
\hline $0250+320$ & $\ldots$ & $\ldots$ & $\ldots$ & 1 & 1 & 1 & $\ldots$ & $\ldots$ & $\ldots$ \\
\hline $0256+192$ & $\ldots$ & $\ldots$ & $\ldots$ & 3 & 1 & 2 & $\ldots$ & $\ldots$ & $\ldots$ \\
\hline $0300+470$ & 2 & 2 & 2 & 10 & 1 & 2 & 4 & 1 & 1 \\
\hline $0306+102$ & $\ldots$ & $\ldots$ & $\ldots$ & 3 & 1 & 2 & 2 & 1 & 1 \\
\hline $0309+411$ & 1 & 1 & 1 & 3 & 2 & 2 & $\ldots$ & $\ldots$ & $\ldots$ \\
\hline $0322+222$ & 1 & 1 & 1 & 3 & 1 & 1 & $\ldots$ & $\cdots$ & $\ldots$ \\
\hline $0333+321$ & $\ldots$ & $\ldots$ & $\ldots$ & 3 & 1 & 2 & 2 & 1 & 1 \\
\hline 0336-019 & $\ldots$ & $\ldots$ & $\ldots$ & 6 & 1 & 3 & 4 & 1 & 2 \\
\hline $0340+362$ & 1 & 2 & 2 & 5 & 1 & 2 & 1 & 1 & 1 \\
\hline $0345+460$ & 1 & 3 & 3 & 6 & 1 & 2 & 1 & 1 & 1 \\
\hline $0346-279$ & 1 & 2 & 2 & 6 & 1 & 2 & 1 & 1 & 1 \\
\hline $0350+465$ & 1 & 2 & 2 & 6 & 1 & 2 & 1 & 1 & 1 \\
\hline $0354+231$ & $\ldots$ & $\ldots$ & $\ldots$ & 3 & 1 & 1 & $\ldots$ & $\ldots$ & $\ldots$ \\
\hline $0358+040$ & 1 & 1 & 1 & 6 & 1 & 3 & 1 & 1 & 1 \\
\hline $0358+210$ & 1 & 1 & 1 & 3 & 1 & 1 & $\ldots$ & $\ldots$ & $\ldots$ \\
\hline $0400+258$ & 1 & 2 & 2 & 6 & 1 & 2 & 2 & 1 & 1 \\
\hline $0402-362$ & $\ldots$ & $\ldots$ & $\ldots$ & 1 & 1 & 1 & $\ldots$ & $\ldots$ & $\ldots$ \\
\hline 0405-331 & $\ldots$ & $\ldots$ & $\ldots$ & 3 & 1 & 2 & $\ldots$ & $\ldots$ & $\ldots$ \\
\hline 0405-385 & $\cdots$ & $\ldots$ & $\ldots$ & 1 & 2 & 2 & $\ldots$ & $\ldots$ & $\ldots$ \\
\hline $0406+121$ & $\ldots$ & $\ldots$ & $\ldots$ & 1 & 2 & 2 & $\ldots$ & $\ldots$ & $\ldots$ \\
\hline $0409+229$ & 1 & 3 & 3 & 2 & 1 & 2 & $\ldots$ & $\ldots$ & $\ldots$ \\
\hline $0415+398$ & $\ldots$ & $\ldots$ & $\ldots$ & 3 & 1 & 2 & $\ldots$ & $\ldots$ & $\ldots$ \\
\hline $0420-014$ & $\ldots$ & $\ldots$ & $\ldots$ & 3 & 1 & 1 & $\ldots$ & $\ldots$ & $\ldots$ \\
\hline $0420+022$ & $\ldots$ & $\ldots$ & $\ldots$ & 3 & 2 & 2 & $\ldots$ & $\ldots$ & $\ldots$ \\
\hline $0422+004$ & $\ldots$ & $\ldots$ & $\ldots$ & 3 & 1 & 1 & $\ldots$ & $\ldots$ & $\ldots$ \\
\hline 0422-380 & $\ldots$ & $\ldots$ & $\ldots$ & 1 & 2 & 2 & $\ldots$ & $\ldots$ & $\ldots$ \\
\hline $0425+048$ & $\ldots$ & $\ldots$ & $\ldots$ & 1 & 1 & 1 & $\ldots$ & $\ldots$ & $\ldots$ \\
\hline $0426+273$ & 1 & 2 & 2 & 3 & 1 & 1 & $\ldots$ & $\ldots$ & $\ldots$ \\
\hline $0426-380$ & $\ldots$ & $\ldots$ & $\ldots$ & 1 & 1 & 1 & $\ldots$ & $\ldots$ & $\ldots$ \\
\hline $0429+174$ & $\ldots$ & $\ldots$ & $\ldots$ & 2 & 1 & 1 & $\ldots$ & $\ldots$ & $\ldots$ \\
\hline $0430+052$ & $\ldots$ & $\ldots$ & $\ldots$ & 3 & 2 & 3 & $\ldots$ & $\ldots$ & $\ldots$ \\
\hline $0446+112$ & 1 & 1 & 1 & 5 & 1 & 2 & 2 & 1 & 1 \\
\hline $0450+013$ & $\ldots$ & $\cdots$ & $\cdots$ & 1 & 1 & 1 & $\ldots$ & $\ldots$ & $\ldots$ \\
\hline $0454-234$ & 2 & 1 & 2 & 10 & 1 & 2 & 4 & 1 & 3 \\
\hline $0458-020$ & 2 & 1 & 2 & 10 & 1 & 1 & 4 & 1 & 1 \\
\hline $0458+138$ & 1 & 2 & 2 & 1 & 1 & 1 & $\ldots$ & $\ldots$ & $\ldots$ \\
\hline
\end{tabular}


TABLE $4-$ Continued

\begin{tabular}{|c|c|c|c|c|c|c|c|c|c|}
\hline \multirow[b]{2}{*}{ Source } & \multicolumn{3}{|c|}{$\mathrm{X}$ band $(8.4 \mathrm{GHz})$} & \multicolumn{3}{|c|}{$\mathrm{K}$ band $(24 \mathrm{GHz})$} & \multicolumn{3}{|c|}{$\mathrm{Q}$ band $(43 \mathrm{GHz})$} \\
\hline & $N_{\text {session }}$ & $S I_{\min }$ & $S I_{\max }$ & $N_{\text {session }}$ & $S I_{\min }$ & $S I_{\max }$ & $N_{\text {session }}$ & $S I_{\min }$ & $S I_{\max }$ \\
\hline $0459+135$ & $\ldots$ & $\ldots$ & $\ldots$ & 3 & 1 & 1 & $\ldots$ & $\ldots$ & $\ldots$ \\
\hline $0502+049$ & $\ldots$ & $\ldots$ & $\ldots$ & 3 & 1 & 2 & $\ldots$ & $\ldots$ & $\ldots$ \\
\hline $0506+056$ & 1 & 2 & 2 & 6 & 1 & 2 & 1 & 1 & 1 \\
\hline $0507+179$ & 1 & 2 & 2 & 3 & 1 & 1 & $\ldots$ & $\ldots$ & $\ldots$ \\
\hline $0511-220$ & $\ldots$ & $\ldots$ & $\ldots$ & 3 & 1 & 2 & $\ldots$ & $\ldots$ & $\ldots$ \\
\hline $0524+034$ & 1 & 1 & 1 & 6 & 1 & 1 & 1 & 1 & 1 \\
\hline $0528+134$ & 1 & 1 & 1 & 5 & 1 & 3 & 2 & 2 & 2 \\
\hline $0528-250$ & $\ldots$ & $\ldots$ & $\ldots$ & 1 & 2 & 2 & $\ldots$ & $\ldots$ & $\ldots$ \\
\hline 0534-340 & $\ldots$ & $\ldots$ & $\ldots$ & 1 & 1 & 1 & $\ldots$ & $\ldots$ & $\ldots$ \\
\hline $0537-441$ & $\ldots$ & $\ldots$ & $\ldots$ & 1 & 2 & 2 & $\ldots$ & $\ldots$ & $\ldots$ \\
\hline $0544+273$ & 1 & 2 & 2 & 4 & 1 & 1 & $\ldots$ & $\ldots$ & $\ldots$ \\
\hline $0547+234$ & 1 & 2 & 2 & 3 & 1 & 3 & $\ldots$ & $\ldots$ & $\ldots$ \\
\hline $0549+192$ & 1 & 2 & 2 & 1 & 1 & 1 & $\ldots$ & $\ldots$ & $\ldots$ \\
\hline $0552+398$ & 1 & 3 & 3 & 5 & 2 & 2 & 2 & 1 & 1 \\
\hline $0554+242$ & 1 & 2 & 2 & 3 & 1 & 3 & $\ldots$ & $\cdots$ & $\ldots$ \\
\hline $0556+238$ & 1 & 1 & 1 & 4 & 1 & 1 & 2 & 1 & 1 \\
\hline $0601+245$ & 1 & 3 & 3 & 1 & 2 & 2 & $\ldots$ & $\ldots$ & $\ldots$ \\
\hline $0607-157$ & 2 & 1 & 2 & 8 & 1 & 3 & 2 & 1 & 2 \\
\hline $0611+131$ & 1 & 1 & 1 & 3 & 1 & 2 & $\ldots$ & $\ldots$ & $\ldots$ \\
\hline $0621+446$ & $\ldots$ & $\ldots$ & $\ldots$ & 1 & 1 & 1 & $\ldots$ & $\ldots$ & $\ldots$ \\
\hline 0639-032 & 1 & 2 & 2 & 4 & 1 & 2 & $\ldots$ & $\ldots$ & $\ldots$ \\
\hline $0639+352$ & & & & 1 & 2 & 2 & $\ldots$ & $\ldots$ & $\ldots$ \\
\hline $0642+449$ & 1 & 1 & 1 & 4 & 2 & 2 & 2 & 2 & 3 \\
\hline 0646-306 & $\ldots$ & $\ldots$ & $\ldots$ & 3 & 2 & 3 & 2 & 1 & 2 \\
\hline $0648-165$ & 2 & 1 & 1 & 10 & 1 & 2 & 4 & 1 & 3 \\
\hline $0654+244$ & 1 & 3 & 3 & 1 & 1 & 1 & $\ldots$ & $\ldots$ & $\ldots$ \\
\hline $0707+476$ & $\ldots$ & $\ldots$ & $\ldots$ & 3 & 1 & 2 & 1 & 3 & 3 \\
\hline $0716+477$ & $\ldots$ & $\ldots$ & $\ldots$ & 3 & 1 & 2 & $\ldots$ & $\ldots$ & $\ldots$ \\
\hline 0721-071 & 1 & 2 & 2 & 4 & 1 & 2 & $\ldots$ & $\ldots$ & $\ldots$ \\
\hline $0722+145$ & 1 & 2 & 2 & 5 & 1 & 1 & 1 & 2 & 2 \\
\hline $0725+219$ & 1 & 2 & 2 & 2 & 1 & 2 & $\ldots$ & $\ldots$ & $\ldots$ \\
\hline $0727-115$ & .. & $\cdots$ & $\cdots$ & 6 & 1 & 2 & 4 & 1 & 2 \\
\hline $0728+249$ & 1 & 2 & 2 & $\ldots$ & $\ldots$ & $\ldots$ & $\ldots$ & $\ldots$ & $\ldots$ \\
\hline $0735+178$ & 1 & 3 & 3 & 6 & 2 & 2 & 2 & 2 & 2 \\
\hline $0736+017$ & $\ldots$ & $\ldots$ & $\ldots$ & 3 & 1 & 2 & $\ldots$ & $\ldots$ & $\ldots$ \\
\hline 0743-006 & 1 & 1 & 1 & 4 & 2 & 4 & 2 & 2 & 2 \\
\hline $0745+241$ & 1 & 2 & 2 & 6 & 1 & 2 & 2 & 1 & 1 \\
\hline $0746+483$ & 1 & 2 & 2 & 6 & 1 & 2 & 1 & 1 & 1 \\
\hline $0748+126$ & 2 & 1 & 1 & 8 & 1 & 2 & 2 & 1 & 1 \\
\hline $0749+540$ & 2 & 2 & 2 & 10 & 1 & 2 & 4 & 1 & 2 \\
\hline $0754+100$ & 2 & 2 & 2 & 10 & 1 & 3 & 4 & 1 & 2 \\
\hline $0759+183$ & 1 & 2 & 2 & 3 & 1 & 1 & $\ldots$ & $\ldots$ & $\ldots$ \\
\hline $0804+499$ & 2 & 1 & 2 & 10 & 1 & 2 & 4 & 1 & 1 \\
\hline 0805-077 & & $\ldots$ & $\ldots$ & 3 & 1 & 2 & 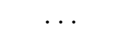 & $\ldots$ & $\cdots$ \\
\hline $0805+410$ & 1 & 1 & 1 & 5 & 1 & 1 & 2 & 1 & 1 \\
\hline $0808+019$ & 2 & 1 & 1 & 10 & 1 & 1 & 4 & 1 & 1 \\
\hline $0812+367$ & & $\ldots$ & $\ldots$ & 3 & 2 & 2 & $\ldots$ & $\ldots$ & \\
\hline $0814+425$ & 1 & 2 & 2 & 5 & 1 & 2 & 2 & 1 & 1 \\
\hline $0820+560$ & 1 & 3 & 3 & 4 & 2 & 2 & 2 & 1 & 1 \\
\hline $0821+248$ & 1 & 1 & 1 & 2 & 1 & 1 & $\ldots$ & $\ldots$ & $\ldots$ \\
\hline $0821+394$ & 1 & 1 & 1 & 3 & 1 & 1 & $\ldots$ & $\ldots$ & $\ldots$ \\
\hline $0823+033$ & 1 & 2 & 2 & 4 & 1 & 2 & 2 & 1 & 2 \\
\hline $0827+243$ & 1 & 2 & 2 & 4 & 1 & 2 & $\ldots$ & $\ldots$ & $\ldots$ \\
\hline 0834-201 & $\ldots$ & $\cdots$ & $\ldots$ & 3 & 2 & 2 & $\ldots$ & $\ldots$ & $\ldots$ \\
\hline $0834+250$ & 1 & 2 & 2 & 3 & 1 & 2 & $\ldots$ & $\ldots$ & $\ldots$ \\
\hline $0838+133$ & 1 & 3 & 3 & 6 & 2 & 2 & 1 & 2 & 2 \\
\hline $0839+187$ & 1 & 4 & 4 & 2 & 1 & 2 & $\cdots$ & $\ldots$ & $\ldots$ \\
\hline $0850+581$ & 1 & 3 & 3 & 2 & 1 & 2 & $\ldots$ & $\ldots$ & $\ldots$ \\
\hline $0851+202$ & 1 & 2 & 2 & 5 & 1 & 2 & 2 & 1 & 2 \\
\hline $0906+163$ & $\ldots$ & $\ldots$ & $\ldots$ & 2 & 1 & 1 & $\ldots$ & $\ldots$ & $\ldots$ \\
\hline $0912+029$ & $\ldots$ & $\ldots$ & $\ldots$ & 3 & 1 & 2 & $\ldots$ & $\ldots$ & $\ldots$ \\
\hline $0917+624$ & 1 & 3 & 3 & 4 & 1 & 2 & 2 & 1 & 2 \\
\hline $0923+392$ & 1 & 3 & 3 & 4 & 3 & 3 & 2 & 4 & 4 \\
\hline $0945+408$ & 1 & 4 & 4 & 3 & 2 & 3 & $\ldots$ & $\cdots$ & $\ldots$ \\
\hline $0953+254$ & 2 & 3 & 3 & 10 & 1 & 2 & 4 & 1 & 2 \\
\hline $0955+326$ & 1 & 2 & 2 & 3 & 2 & 2 & $\ldots$ & $\ldots$ & $\ldots$ \\
\hline $0955+476$ & $\ldots$ & $\ldots$ & $\ldots$ & 2 & 2 & 2 & 1 & 1 & 1 \\
\hline $1005+066$ & $\ldots$ & $\ldots$ & $\ldots$ & 3 & 1 & 2 & $\ldots$ & $\ldots$ & $\ldots$ \\
\hline $1012+232$ & $\ldots$ & $\ldots$ & $\ldots$ & 3 & 2 & 3 & $\ldots$ & $\ldots$ & $\ldots$ \\
\hline 1016-311 & $\cdots$ & $\ldots$ & $\ldots$ & 2 & 2 & 2 & $\ldots$ & $\ldots$ & $\ldots$ \\
\hline $1020+400$ & $\ldots$ & $\ldots$ & $\ldots$ & 3 & 1 & 2 & $\ldots$ & $\ldots$ & $\ldots$ \\
\hline $1022+194$ & 1 & 2 & 2 & 3 & 1 & 1 & $\ldots$ & $\ldots$ & $\ldots$ \\
\hline $1022+237$ & $\ldots$ & $\ldots$ & $\ldots$ & 1 & 1 & 1 & $\ldots$ & $\ldots$ & $\ldots$ \\
\hline $1032-199$ & $\ldots$ & $\ldots$ & $\ldots$ & 3 & 1 & 3 & $\ldots$ & $\ldots$ & $\ldots$ \\
\hline $1034-293$ & 2 & 2 & 3 & 9 & 1 & 2 & 3 & 1 & 3 \\
\hline $1039+811$ & $\ldots$ & $\ldots$ & $\ldots$ & 3 & 1 & 2 & 1 & 1 & 1 \\
\hline
\end{tabular}


TABLE $4-$ Continued

\begin{tabular}{|c|c|c|c|c|c|c|c|c|c|}
\hline \multirow[b]{2}{*}{ Source } & \multicolumn{3}{|c|}{$\mathrm{X}$ band $(8.4 \mathrm{GHz})$} & \multicolumn{3}{|c|}{$\mathrm{K}$ band $(24 \mathrm{GHz})$} & \multicolumn{3}{|c|}{$\mathrm{Q}$ band $(43 \mathrm{GHz})$} \\
\hline & $N_{\text {session }}$ & $S I_{\min }$ & $S I_{\max }$ & $N_{\text {session }}$ & $S I_{\min }$ & $S I_{\max }$ & $N_{\text {session }}$ & $S I_{\min }$ & $S I_{\max }$ \\
\hline $1045-188$ & 2 & 2 & 3 & 10 & 1 & 2 & 4 & 1 & 2 \\
\hline $1048-313$ & & $\cdots$ & $\cdots$ & 1 & 3 & 3 & $\cdots$ & $\cdots$ & $\cdots$ \\
\hline $1049+215$ & & $\cdots$ & $\cdots$ & 3 & 1 & 2 & 1 & 1 & 1 \\
\hline $1053+815$ & 2 & 2 & 3 & 7 & 1 & 1 & 2 & 1 & 1 \\
\hline $1055+018$ & 1 & 2 & 2 & 8 & 1 & 2 & 3 & 2 & 2 \\
\hline $1101+384$ & & $\cdots$ & $\cdots$ & 3 & 1 & 1 & $\cdots$ & & \\
\hline $1113+087$ & & 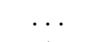 & & 2 & 2 & 2 & $\cdots$ & .. & .. \\
\hline $1124-186$ & 2 & 1 & 1 & 10 & 1 & 1 & 4 & 1 & 2 \\
\hline $1128+385$ & & .. & $\cdots$ & 3 & 1 & 2 & 1 & 1 & 1 \\
\hline $1142+052$ & & & . & 2 & 1 & 3 & ... & & \\
\hline $1144-379$ & 1 & 1 & 1 & 1 & 2 & 2 & .. & 3. & .. \\
\hline $1144+402$ & & & & 5 & 1 & 1 & 2 & 1 & 1 \\
\hline $1147+245$ & 1 & 2 & 2 & 3 & 1 & 2 & $\cdots$ & $\cdots$ & $\cdots$ \\
\hline $1150+497$ & & & & 3 & 2 & 2 & $\cdots$ & $\cdots$ & $\cdots$ \\
\hline $1150+812$ & 1 & 3 & 3 & 3 & 3 & 3 & $\cdots$ & $\cdots$ & $\cdots$ \\
\hline $1156+295$ & 1 & 1 & 1 & 5 & 1 & 2 & 1 & 1 & 1 \\
\hline 1207-319 & & & $\cdots$ & 1 & 2 & 2 & .. & $\cdots$ & ... \\
\hline $1213-172$ & $\cdots$ & $\ldots$ & $\cdots$ & 3 & 2 & 2 & $\cdots$ & ... $>$ & $\cdots$ \\
\hline $1236+077$ & & $\ldots$ & $\cdots$ & 3 & 1 & 1 & 1 & 3 & 3 \\
\hline $1243-072$ & & & & 3 & 1 & 1 & 1 & 1 & 1 \\
\hline $1256-220$ & 1 & 1 & 1 & 6 & 1 & 2 & 1 & 1 & 1 \\
\hline $1302-102$ & 1 & & $\ldots$ & 1 & 2 & 2 & 1 & 1 & 1 \\
\hline $1308+326$ & 2 & 2 & 3 & 10 & 1 & 2 & 4 & 1 & 2 \\
\hline $1308+554$ & 1 & 2 & 2 & 3 & 1 & 1 & & & \\
\hline $1313-333$ & 1 & 3 & 3 & 5 & 1 & 3 & 2 & 2 & 2 \\
\hline $1324+224$ & 1 & 1 & 1 & 5 & 1 & 2 & 2 & 1 & 1 \\
\hline $1334-127$ & 1 & 2 & 2 & 5 & 1 & 2 & 2 & 1 & 1 \\
\hline $1354-152$ & $\ldots$ & $\ldots$ & $\ldots$ & 3 & 1 & 1 & 1 & 2 & 2 \\
\hline $1357+769$ & $\cdots$ & $\ldots$ & $\cdots$ & 2 & 1 & 1 & 1 & 1 & 1 \\
\hline 1406-076 & $\cdots$ & $\cdots$ & $\cdots$ & 2 & 1 & 2 & 1 & 2 & 2 \\
\hline 1406-267 & 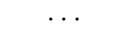 & $\ldots$ & .. & 1 & 2 & 2 & $\cdots$ & 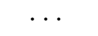 & $\ldots$ \\
\hline $1418+546$ & 1 & 3 & 3 & 3 & 2 & 2 & $\ldots$ & $\ldots$ & $\ldots$ \\
\hline $1435-218$ & & & & 3 & 1 & 2 & $\ldots$ & $\ldots$ & $\ldots$ \\
\hline $1437-153$ & $\ldots$ & $\ldots$ & $\ldots$ & 3 & 1 & 1 & $\ldots$ & $\ldots$ & $\ldots$ \\
\hline $1451-375$ & $\ldots$ & $\ldots$ & $\ldots$ & 1 & 1 & 1 & $\ldots$ & $\ldots$ & $\ldots$ \\
\hline $1459+480$ & $\ldots$ & $\ldots$ & $\ldots$ & 3 & 1 & 2 & $\ldots$ & $\ldots$ & $\ldots$ \\
\hline $1502+036$ & & & & 2 & 1 & 1 & 1 & 2 & 2 \\
\hline $1502+106$ & 2 & 2 & 2 & 10 & 1 & 2 & 4 & 1 & 2 \\
\hline $1504+377$ & 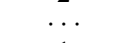 & $\ldots$ & $\ldots$ & 1 & 2 & 2 & $\cdots$ & $\cdots$ & $\ldots$ \\
\hline $1505+428$ & 1 & 3 & 3 & 6 & 1 & 2 & 1 & 2 & 2 \\
\hline 1511-100 & 1 & 3 & 3 & 6 & 1 & 2 & 3 & 1 & 2 \\
\hline $1514+004$ & 1 & 3 & 3 & 5 & 2 & 2 & 1 & 2 & 2 \\
\hline $1514+197$ & & & & 3 & 1 & 1 & & & \\
\hline $1514-241$ & $\cdots$ & $\ldots$ & $\cdots$ & 3 & 2 & 3 & $\cdots$ & $\cdots$ & $\ldots$ \\
\hline $1519-273$ & $\ldots$ & $\ldots$ & $\ldots$ & 3 & 1 & 1 & $\ldots$ & $\ldots$ & $\ldots$ \\
\hline $1520+437$ & 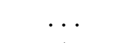 & 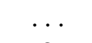 & 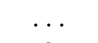 & 1 & 1 & 1 & $\ldots$ & $\ldots$ & $\ldots$ \\
\hline $1546+027$ & 1 & 2 & 2 & 5 & 1 & 2 & 2 & 1 & 1 \\
\hline $1548+056$ & 1 & 3 & 3 & 4 & 1 & 3 & $\ldots$ & $\ldots$ & $\ldots$ \\
\hline $1606+106$ & 1 & 3 & 3 & 5 & 1 & 2 & 2 & 1 & 2 \\
\hline $1611+343$ & 1 & 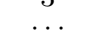 & 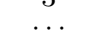 & 3 & 2 & 2 & 1 & 1 & 1 \\
\hline $1617+229$ & 1 & 2 & 2 & 6 & 1 & 2 & 1 & 1 & 1 \\
\hline $1622-253$ & $\ldots$ & $\ldots$ & $\ldots$ & 3 & 1 & 1 & $\ldots$ & $\cdots$ & $\cdots$ \\
\hline $1622-297$ & & .. & $\cdots$ & 3 & 1 & 2 & $\cdots$ & & $\cdots$ \\
\hline $1637+574$ & 1 & 1 & 1 & 4 & 1 & 2 & 2 & 2 & 2 \\
\hline $1638+398$ & 1 & 1 & 1 & 5 & 1 & 2 & 2 & 1 & 1 \\
\hline $1652+398$ & & & & 3 & 2 & 2 & & & \\
\hline $1656-075$ & $\cdots$ & $\ldots$ & $\cdots$ & 3 & 1 & 3 & $\cdots$ & $\cdots$ & $\ldots$ \\
\hline $1657-261$ & $\ldots$ & $\ldots$ & $\ldots$ & 3 & 1 & 1 & $\ldots$ & $\ldots$ & $\ldots$ \\
\hline $1705+018$ & $\ldots$ & $\ldots$ & $\ldots$ & 3 & 1 & 1 & 1 & 1 & 1 \\
\hline $1710-269$ & $\ldots$ & $\ldots$ & $\ldots$ & 2 & 2 & 3 & $\ldots$ & + & \\
\hline $1717+178$ & $\ldots$ & $\ldots$ & $\ldots$ & 3 & 2 & 2 & 1 & 1 & 1 \\
\hline $1726+455$ & 2 & 1 & 2 & 10 & 1 & 1 & 4 & 1 & 3 \\
\hline $1730-130$ & 2 & 2 & 2 & 9 & 1 & 2 & 3 & 1 & 2 \\
\hline $1732+389$ & 1 & 1 & 1 & 5 & 1 & 2 & 2 & 2 & 2 \\
\hline $1738+476$ & & 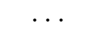 & $\cdots$ & 3 & 1 & 2 & 1 & 1 & 1 \\
\hline $1741-038$ & 1 & 1 & 1 & 5 & 1 & 3 & 3 & 1 & 2 \\
\hline $1741-312$ & & $\cdots$ & $\cdots$ & 1 & 3 & 3 & $\cdots$ & $\cdots$ & $\cdots$ \\
\hline $1742-078$ & 1 & 3 & 3 & 6 & 2 & 2 & 1 & 1 & 1 \\
\hline $1749+096$ & 1 & 1 & 1 & 5 & 1 & 2 & 2 & 1 & 1 \\
\hline $1749+701$ & 1 & 3 & 3 & 3 & 2 & 3 & & & \\
\hline $1751+288$ & & & $\ldots$ & 3 & 1 & 1 & 1 & 1 & 1 \\
\hline $1754+155$ & ... & $\ldots$ & $\ldots$ & 3 & 1 & 2 & & & \\
\hline $1800+440$ & & $\ldots$ & $\ldots$ & 3 & 1 & 1 & $\cdots$ & $\ldots$ & $\ldots$ \\
\hline $1803+784$ & 1 & 3 & 3 & 4 & 1 & 2 & 2 & 1 & 2 \\
\hline $1817-254$ & & $x_{0}$ & $\ldots$ & 2 & 1 & 1 & $\cdots$ & $\ldots$ & $\cdots$ \\
\hline $1817+387$ & & $\ldots$ & ... & 3 & 1 & 3 & $\ldots$ & & $\ldots$ \\
\hline
\end{tabular}


TABLE $4-$ Continued

\begin{tabular}{|c|c|c|c|c|c|c|c|c|c|}
\hline \multirow[b]{2}{*}{ Source } & \multicolumn{3}{|c|}{$\mathrm{X}$ band $(8.4 \mathrm{GHz})$} & \multicolumn{3}{|c|}{$\mathrm{K}$ band $(24 \mathrm{GHz})$} & \multicolumn{3}{|c|}{$\mathrm{Q}$ band $(43 \mathrm{GHz})$} \\
\hline & $N_{\text {session }}$ & $S I_{\min }$ & $S I_{\max }$ & $N_{\text {session }}$ & $S I_{\min }$ & $S I_{\max }$ & $N_{\text {session }}$ & $S I_{\min }$ & $S I_{\max }$ \\
\hline $1829-106$ & $\ldots$ & $\ldots$ & $\ldots$ & 2 & 2 & 3 & $\ldots$ & $\ldots$ & $\ldots$ \\
\hline $1829-207$ & $\ldots$ & $\ldots$ & $\ldots$ & 2 & 2 & 3 & $\ldots$ & $\ldots$ & $\ldots$ \\
\hline $1830-211$ & $\ldots$ & $\ldots$ & $\ldots$ & 2 & 2 & 2 & $\ldots$ & $\ldots$ & $\ldots$ \\
\hline $1849+670$ & 1 & 1 & 1 & 4 & 1 & 1 & 2 & 1 & 1 \\
\hline $1901+319$ & $\ldots$ & $\ldots$ & $\cdots$ & 3 & 1 & 3 & 2 & 1 & 1 \\
\hline 1921-293 & 2 & 2 & 3 & 8 & 1 & 2 & 3 & 1 & 2 \\
\hline $1928+154$ & $\ldots$ & $\ldots$ & $\cdots$ & 3 & 1 & 2 & $\ldots$ & $\cdots$ & ... \\
\hline $1936-155$ & 1 & 1 & 1 & 3 & 1 & 1 & 2 & 1 & 2 \\
\hline $1946-200$ & $\ldots$ & $\ldots$ & $\cdots$ & 1 & 2 & 2 & $\ldots$ & $\ldots$ & $\ldots$ \\
\hline $1954-388$ & $\ldots$ & $\ldots$ & $\ldots$ & 1 & 1 & 1 & $\ldots$ & $\ldots$ & $\ldots$ \\
\hline $1958-179$ & $\ldots$ & $\ldots$ & $\ldots$ & 2 & 1 & 1 & 1 & 1 & 1 \\
\hline $2000+472$ & $\ldots$ & $\ldots$ & $\ldots$ & 3 & 1 & 2 & $\ldots$ & $\ldots$ & $\ldots$ \\
\hline 2008-159 & 2 & 1 & 1 & 10 & 1 & 2 & 4 & 1 & 2 \\
\hline $2013+163$ & $\ldots$ & $\ldots$ & $\ldots$ & 3 & 1 & 1 & $\ldots$ & $\ldots$ & $\ldots$ \\
\hline $2021+317$ & $\ldots$ & $\ldots$ & $\ldots$ & 3 & 2 & 3 & 2 & 1 & 1 \\
\hline $2029+121$ & $\ldots$ & $\ldots$ & $\ldots$ & 1 & 2 & 2 & $\ldots$ & $\ldots$ & $\ldots$ \\
\hline $2037+511$ & $\ldots$ & $\ldots$ & $\ldots$ & 3 & 1 & 2 & 1 & 1 & 1 \\
\hline $2048+312$ & 1 & 3 & 3 & 3 & 1 & 3 & $\ldots$ & $\ldots$ & $\ldots$ \\
\hline $2054-377$ & $\ldots$ & $\ldots$ & $\ldots$ & 1 & 1 & 1 & $\ldots$ & $\ldots$ & $\ldots$ \\
\hline $2106-413$ & $\ldots$ & $\ldots$ & $\ldots$ & 1 & 2 & 2 & $\ldots$ & $\ldots$ & $\ldots$ \\
\hline $2113+293$ & $\ldots$ & $\ldots$ & $\ldots$ & 3 & 2 & 2 & 1 & 1 & 1 \\
\hline $2121+053$ & 1 & 1 & 1 & 5 & 1 & 2 & 2 & 1 & 1 \\
\hline $2126-158$ & $\ldots$ & $\ldots$ & $\ldots$ & 3 & 1 & 3 & 1 & 3 & 3 \\
\hline $2128-123$ & $\ldots$ & $\ldots$ & $\ldots$ & 3 & 3 & 4 & $\ldots$ & $\cdots$ & $\ldots$ \\
\hline 2131-021 & 1 & 3 & 3 & 5 & 2 & 2 & 2 & 2 & 2 \\
\hline $2136+141$ & 2 & 2 & 2 & 10 & 2 & 3 & 4 & 1 & 1 \\
\hline 2143-156 & 1 & 2 & 2 & 3 & 1 & 1 & ... & $\ldots$ & $\ldots$ \\
\hline $2145+067$ & 1 & 3 & 3 & 5 & 1 & 2 & 2 & 2 & 2 \\
\hline $2150+173$ & 1 & 3 & 3 & 4 & 2 & 2 & 1 & 1 & 1 \\
\hline $2200+420$ & $\ldots$ & $\ldots$ & $\ldots$ & 3 & 1 & 2 & 2 & 1 & 1 \\
\hline $2201+171$ & $\cdots$ & $\cdots$ & $\cdots$ & 3 & 1 & 2 & $\cdots$ & $\cdots$ & $\cdots$ \\
\hline $2209+236$ & $\ldots$ & $\ldots$ & $\ldots$ & 3 & 1 & 2 & $\ldots$ & $\ldots$ & $\ldots$ \\
\hline $2214+350$ & $\ldots$ & $\ldots$ & $\ldots$ & 3 & 1 & 2 & $\ldots$ & $\ldots$ & $\ldots$ \\
\hline $2227-088$ & 1 & 1 & 1 & 9 & 1 & 1 & 4 & 1 & 1 \\
\hline $2239+096$ & 1 & 2 & 2 & 6 & 1 & 2 & 1 & 2 & 2 \\
\hline $2251+158$ & 1 & 4 & 4 & 3 & 2 & 2 & $\ldots$ & $\ldots$ & $\ldots$ \\
\hline $2254+024$ & $\ldots$ & $\ldots$ & $\ldots$ & 3 & 1 & 1 & $\ldots$ & $\ldots$ & $\ldots$ \\
\hline $2255-282$ & 2 & 1 & 2 & 10 & 1 & 2 & 4 & 1 & 2 \\
\hline $2306+095$ & $\ldots$ & $\ldots$ & $\ldots$ & 1 & 2 & 2 & $\cdots$ & $\ldots$ & $\ldots$ \\
\hline $2309+454$ & 1 & 2 & 2 & 6 & 1 & 2 & 1 & 1 & 1 \\
\hline $2318+049$ & 1 & 1 & 1 & 5 & 1 & 1 & 2 & 1 & 1 \\
\hline $2325+093$ & 1 & 1 & 1 & 6 & 1 & 1 & 1 & 1 & 1 \\
\hline $2335-027$ & $\ldots$ & $\ldots$ & $\cdots$ & 3 & 1 & 1 & $\ldots$ & $\ldots$ & $\ldots$ \\
\hline $2344+092$ & $\ldots$ & $\ldots$ & $\ldots$ & 3 & 2 & 2 & $\ldots$ & $\ldots$ & $\ldots$ \\
\hline $2346+385$ & $\ldots$ & $\ldots$ & $\ldots$ & 3 & 1 & 2 & $\ldots$ & $\ldots$ & $\ldots$ \\
\hline $2353+816$ & 1 & 2 & 2 & 6 & 1 & 1 & 1 & 1 & 1 \\
\hline $2355-106$ & $\ldots$ & $\ldots$ & $\ldots$ & 3 & 1 & 1 & $\ldots$ & $\ldots$ & $\ldots$ \\
\hline $2357-318$ & $\ldots$ & $\ldots$ & $\ldots$ & 3 & 1 & 1 & $\ldots$ & $\ldots$ & $\ldots$ \\
\hline
\end{tabular}


The Celestial Reference Frame at 24 and $43 \mathrm{GHz}$

TABLE 5

Source COMPACTNESS VS. SOURCE STRUCTURE INDEX.

\begin{tabular}{lccccccc}
\hline \hline & \multicolumn{3}{c}{ K Band $(24 \mathrm{GHz})$} & & \multicolumn{3}{c}{ Q Band (43 GHz) } \\
\cline { 2 - 4 } Struc. Ind. & $N_{\text {sou }}$ & Mean & Median & & $N_{\text {sou }}$ & Mean & Median \\
\hline $1 \ldots \ldots \ldots$ & 84 & 0.89 & 0.90 & & 74 & 0.87 & 0.87 \\
$2 \ldots \ldots \ldots$ & 131 & 0.79 & 0.80 & & 48 & 0.74 & 0.75 \\
$3 \ldots \ldots \ldots$ & 40 & 0.70 & 0.70 & & 9 & 0.71 & 0.71 \\
$4 \ldots \ldots \ldots$ & $\ldots$ & $\ldots$ & $\ldots$ & & $\ldots$ & $\cdots$ & $\cdots$ \\
\hline
\end{tabular}

TABLE 6

Source POSITION UNCERTAINTIES VS. SOURCE STRUCTURE INDEX.

\begin{tabular}{|c|c|c|c|c|c|c|c|c|c|c|}
\hline \multirow[b]{3}{*}{ Struc. Ind. } & \multicolumn{5}{|c|}{$\mathrm{X}$ band $(8.4 \mathrm{GHz})^{\mathrm{a}}$} & \multicolumn{5}{|c|}{$\mathrm{K}$ Band $(24 \mathrm{GHz})^{\mathrm{b}}$} \\
\hline & \multirow[b]{2}{*}{$N_{\text {sou }}$} & \multicolumn{2}{|c|}{ Mean (mas) } & \multicolumn{2}{|c|}{ Median (mas) } & \multirow[b]{2}{*}{$N_{\text {sou }}$} & \multicolumn{2}{|c|}{ Mean (mas) } & \multirow{2}{*}{$\begin{array}{l}\text { Median } \\
\alpha \cos \delta\end{array}$} & \multirow{2}{*}{$\frac{(\mathrm{mas})}{\delta}$} \\
\hline & & $\alpha \cos \delta$ & $\delta$ & $\alpha \cos \delta$ & $\delta$ & & $\alpha \cos \delta$ & $\delta$ & & \\
\hline $1 \ldots \ldots \ldots$ & 50 & 0.34 & 0.42 & 0.28 & 0.31 & 49 & 0.27 & 0.33 & 0.27 & 0.31 \\
\hline $2 \ldots \ldots \ldots$ & 106 & 0.38 & 0.45 & 0.29 & 0.32 & 119 & 0.28 & 0.33 & 0.27 & 0.30 \\
\hline $3 \ldots \ldots \ldots$ & 72 & 0.44 & 0.54 & 0.33 & 0.36 & 23 & 0.27 & 0.32 & 0.27 & 0.30 \\
\hline $4 \ldots \ldots$ & 36 & 0.54 & 0.72 & 0.76 & 1.07 & $\cdots$ & $\ldots$ & $\ldots$ & $\ldots$ & $\ldots$ \\
\hline
\end{tabular}

${ }^{\mathrm{a}} \mathrm{X}$ band ICRF values taken from Fey \& Charlot 2000)

b $\mathrm{K}$ band formal errors used to compute the mean and median uncertainties were inflated so as to be comparable to the X-band ICRF values (see discussion in the text). 